\title{
Distortion product otoacoustic emissions from the anuran inner ear
}

Citation for published version (APA):

Meenderink, S. W. F. (2005). Distortion product otoacoustic emissions from the anuran inner ear. [Doctoral Thesis, Maastricht University]. Datawyse / Universitaire Pers Maastricht. https://doi.org/10.26481/dis.20050609sm

Document status and date:

Published: 01/01/2005

DOI:

10.26481/dis.20050609sm

Document Version:

Publisher's PDF, also known as Version of record

\section{Please check the document version of this publication:}

- A submitted manuscript is the version of the article upon submission and before peer-review. There can be important differences between the submitted version and the official published version of record.

People interested in the research are advised to contact the author for the final version of the publication, or visit the DOI to the publisher's website.

- The final author version and the galley proof are versions of the publication after peer review.

- The final published version features the final layout of the paper including the volume, issue and page numbers.

Link to publication

\footnotetext{
General rights rights.

- You may freely distribute the URL identifying the publication in the public portal. please follow below link for the End User Agreement:

www.umlib.nl/taverne-license

Take down policy

If you believe that this document breaches copyright please contact us at:

repository@maastrichtuniversity.nl

providing details and we will investigate your claim.
}

Copyright and moral rights for the publications made accessible in the public portal are retained by the authors and/or other copyright owners and it is a condition of accessing publications that users recognise and abide by the legal requirements associated with these

- Users may download and print one copy of any publication from the public portal for the purpose of private study or research.

- You may not further distribute the material or use it for any profit-making activity or commercial gain

If the publication is distributed under the terms of Article $25 \mathrm{fa}$ of the Dutch Copyright Act, indicated by the "Taverne" license above, 


\section{Distortion product otoacoustic emissions from the anuran inner ear}


(C) S.W.F. Meenderink, Maastricht 2005

Universitaire Pers Maastricht

ISBN 9052784612 


\section{Distortion product otoacoustic emissions from the anuran inner ear}

\section{PROEFSCHRIFT}

ter verkrijging van de graad van doctor aan de Universiteit Maastricht, op gezag van de Rector Magnificus, Prof. Mr. G.P.M.F. Mols, volgens het besluit van het College van Decanen, in het openbaar te verdedigen op donderdag 9 juni 2005 om 16.00 uur door

Sebastiaan Willem Frederik Meenderink

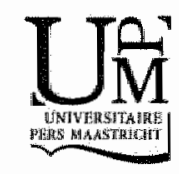




\section{Promotores:}

Prof. Dr. J.J. Manni

Prof. Dr. P. van Dijk (Rijksuniversiteit Groningen)

\section{Beoordelingscommissie:}

Prof. Dr. H.W.M. Stembusch (voorzitter)

Prof. Dr. H. Kingma

Prof. Dr. P.M. Narins (University of California, Los Angeles) 
"Lasciate ogni speranza, voi ch"entrate" Dante, Inferno $1 / 1.9$

"And the frogs shall come up both on thee, and upon thy people, and upon all thy scrvants." EX. $8: 4$ 
The experiments presented in this thesis were performed at the Maastricht University (Chapters 2, 3 and 4) and the University of California, Los Angeles (Chapters 1 and 5).

This work was supported by the Netherlands Organization for Scientific Research (NWO) and the Heinsius Houbolt Foundation. 


\section{Contents}

1 Detailed $f_{1}, f_{2}$ area study of distortion product otoacoustic emissions in the frog.

S.W.F. Meenderink, P.M. Narins, and P. Van Dijk, J. Assoc. Res. Otolaryngol. $6,37-47$

2 Level dependence of distortion product otoacoustic emissions in the leopard frog, Rana pipiens pipiens.

S.W.F. Meenderink and P. van Dijk, Hear. Res. 192, 107-118

3 Characteristics of distortion product otoacoustic emissions in the frog from $L_{1}, L_{2}$-maps.

S.W.F. Meenderink and P. van Dijk, J. Acoust. Soc. Am., accepted after revision

4 The effect of body-temperature on distortion product otoacoustic emissions in the frog.

S.W.F. Meenderink and P. van Dijk

5 Comparison between distortion product otoacoustic emissions and nerve fiber responses from the basilar papilla of the frog.

S.W.F. Meenderink, P. van Dijk, and P.M. Narins, J. Acoust. Soc. Am., accepted after revision

Discussion

Bibliography

Summary in Dutch

Curriculum vitae 


\section{Introduction}

Life dwells in a wondrous, yeit dangerous world. In order to (adequately) interact with, and subsequently respond to this world it is necessary to have a notion of its appearance. Information about the world, and our position within it, comes in a range of different forms, from electromagnetic radiation and chemicals to various forms of mechanical perturbations. Different sensory systems provide the means for perceiving this information, thus allowing the build-up of a mental picture of the world. But if our mental picture of the world composed of light, of sound or of motion-were distorted significantly by the very act of perceiving or thinking about it, then our chances of survival would be negligible. The notion that the evolution of the sensory systems is driven by the necessity to truthfully depict the world around us may explain why widely varying species seem to have similar perception of their surroundings: we all life in the same physical world. This similarity across species is not necessarily reflected in similar anatomical or physiological properties of their sensory organs. Rather, the similarities are to be sought in the functional outputi of these systems, thus providing an unbiased representation of the physical properties that describe the outside world.

With particular reference to the sensory system for the perception of sound, the pressure behind its evolution seems to be the ability to identify, localize and segregate individual sound sources within the surrounclings, a concept known as "auditory scene analysis" (Bregman, 1990). Within this concept, the only constraints on the development of any species' anditory system are simply the hacts of physics, $x^{2}$. those properties of sound that make one sound source different from another (Fay and Popper, 2000).

With respect to the physical properties of sound, one of the major tasks of the peripheral auditory system is the spectral decomposition (i.e. frequency analysis) of sound. This function of the peripheral ear was already suggested by Ohm in 1843 (see Tonndorf (1981) for a historical overview) even before detailed knowledge about its anatomy or functioning was available. Nowadays, detalled data are available about the anatomy, physiology and functioning of the various types of peripheral auditory systems found across vertebrates. Based on this knowledge, several frequency-selective mechanisms (mechanical vs. electrical) that operate at different locations (middle vs. inner ear) and at different scales (micromechanical vs. macromechanical) in the auditory organs of vertebrates have been identified (see Manley (1986) or Patuzzi (1996) for a review of these tuning-mechanisms). These different tuning-mechanisms are thought to give rise to exotic phenomena like 
"traveling waves" (Von Békésy, 1960), "the cochlear amplifier" (Davis, 1983) and "otoacoustic emissions" (Kemp, 1978). Hence, the study of these phenomena may elucidate the mechanisms of frequency-selectivity from which they originate.

In this thesis, If focus on the latter phenomenon, otoncoustic emissions (OAFs). These are sounds generated and emitted by the ear itself. They arise either spontaneously (SOAEs; Kemp, 1979), or during acoustic stimulation (evoked-OAEs; Kemp, 1978) and can be recorded by connecting a sensitive microphone to the ear. It is currently well established that OAE-generation is closely associated with the normal functioning of the inner ear (e.g. Zwicker, 1983); virtually no hearing-impaired ears prodnce otoacoustic emissions. Further, the identification of various types of OAEs in all tetrapod vertelorates, i.e. mammals (Brown, 1987), birds (Froymovich et al, 1995), reptiles (Manley et al., 1993) and amphibians (Van Dijk and Manley, 2001), indicates that their generation reflects fundamental properties of auditory processing. Although this establishes the relation between OAEs and hearing, it does not explain the function of emissions. In fact, it is nowadays generally accepted that OAEs by themselves have no function. Rather, OAEs are a by-product of auditory processing and are considered as evidence for the presence of an active filtering mechanism within the inner ear. Such a mechanism is thought to boost the soundinduced vilorations of the inner ear structures, thus counter-acting the dampening by the inner-ear fluids (Gold, 1948). Since dampening drains energy from the incoming signal, mechanisms that counter-act its effects must supply additional energy, hence the term active amplifier.

In all vertebrates, hair cells are at the basis of sound detection. These cells have stereovilli on their apical-surface, which look like small hairs. Sound-induced deflection of the stereovilli results in an electrical response of the cell, which eventually leads to electrical discharges in the neurons that contact the cell. It is currently believed that the active filtering mechanism also resides in these hair cells. By converting electrochemical energy into mechanical movement; hair cells provide additional energy to the sound-induced vibrations of the inner ear structures on a cycle-by-cycle basis. In a sense, the inner ear sound-detectors increase their own functionality by amplifying the sounds they are to detect.

Since the so-called "cochlear amplifier" (Davis, 1983) resides in hair cells, and hair cells are found in the inner ears of all tetrapod vertebrate classes, it seems that any species can be used to study the nonlinear mechanisms that underlie OAEgeneration. However, across vertebrate classes, hair cells are contained in inner ears that are highly variable in gross anatomy. Since OAEs are recorded outside the ear, they represent the in totu response of the peripheral auditory system. The variation in the gross anatomy of peripheral vertebrate ears will have variable influences 
on the properties of OAEs. In effect, the variation of OAE-characteristics across species does not necessarily arise from variations in the OAE-generation mechamism, but may also result from differences in the conduction of sound to and from the location of OAF-generation. The influence of the gross inner ear anatomy may (seriously) hinder the interpretation of OAEs, becanse it may be unclear whether OAE-characteristics should be attributed to their generation-mechanism or to other tuning-mechanisms.

In frogs, the anatomy of the inner ear is relatively simple. Instead of a cochlea (such as in mammals), the frog ear contains three sensory organs that are sensitive to airborne sound. See Wever (1985) or Lewis and Narins (1999) for extensive descriptions of the anatomy of the anuran peripheral ear. Ignoring the sacculus (which only responds to high-intensity, low-frequency sounds), the remaining two organs are the amphibian papila (AP) and the basilar papilla (BP). The AP is mast sensitive to low- and mid-frequencies within the anuran hearing range. Its sensory epithelium consists of an elongated strip of hair cells. Rather than on a flexible membrane (as in the mammalian cochlea), these cells are embedded in the rigid wall of the papillar recess. Overlying the hair cel]s is a tectorial membrane. Both the shape and the position of this membrane suggest an important role in the transduction of sound by hair cells. The papilla exhibits tonotopic organization with the lowest $(\approx 0.1 \mathrm{kHz})$ and the highest $(\approx 1 \mathrm{kFz})$ frequencies being represented rostrally and caudally, respectively.

The basilar papilla (BP) is most sensitive to the highest frequencies within the hearing range of frogs. In the Northern leopard frog, Rana pipiens pipiens, it responds best to sounds of $1.2-2.4 \mathrm{kHz}$. The anatomy of the BP is unique among all classes of vertebrates. The sensory epithelium holds between 50 and 100 hair cells that stand directly on the rigid cartilaginons wall of the papillat recess. Nearly all of these hair cells are covered by a tectorial membrane. In individual animals, alnost all BP nerve fibers are similarly tuned, with similar-shaped tuning curves and characteristic frequencies that are essentially identical across nerve fbers. Also, afferent newe fibers are not suppressible and their characteristic frequencies do not wary with temperature, indicating that frequency-tuning is of mechanical origin. Fissentially, the BP functions as a single auditory filter with its tuning (presumably) arising from a mechanical filter. The relatively simple anatomy and physiology of these two papillae make the frog an excellent candidate for research on OAEs. The confounding effects of the gross anatomy of the anuran peripheral ear on OAE-characteristics will be largely absent, or at least simpler than those arising from the more elaborate inner ear structures present in other vertebrates.

Since their initial discovery, various types of evoked-OAEs have been identified based on the stimulus required to evoke them. When two pure tones of appropriate 
frequencies $f$ and intensities $L$ are presented to the (amuran) ear, so-called distortion product ctoacoustic emissions (DPOAEs) can be recorded. The four parameters that define the two stimulus tones are generally denoted as $f_{1}, L_{1}$ and $f_{2}, L_{2}$ with the convention that stmulus frequency $f_{2}$ is larger than stimulus frequency $f_{1}$, i.e. $f_{2} / f_{1}>1$. The DPOAFs are frequency components in the recorded signal that are not present in the evoking stimulus. Their frequenctes are given by $f_{\text {dpone }}=$ $a \cdot f_{1}-b \cdot f_{2}$, where $a$ and $b$ are integers. Most commonly studied are the emission components at frequencies $f_{\text {dpone }}=2 f_{1}-f_{2}$ and $2 f_{2}-f_{1}$.

This: thesis deals with distortion product otoacoustic emissions from the inner ear of the Northen leopard frog (Rana pipiens pipiens). Chapters 1,2 and 3 describe the dependence of DPOAEs on the stimulus parameters by exploring various slices/planes within the fou-dimensional parameter-space that defines all combinations of the two stimulus tones. Chapter 1 describes the dependence of DPOAE amplitude and phase on stimulus frequency. By systematically warying $f_{1}$, whille keeping $f_{2} / f_{1}$ fxed, a so-called DPOAE-audiogram is obtained. From hiterature it was already known (Van Dijk and Manley, 2001) that both auditory papillae in the frog ear generate DPOAEs, resulting in two relative amplitude maxima (at different frequencies ) in individual DPOAE audiograms. The combination of a series of DPOAE-audiograms, each one obtained with a different stimulus frequency ratio $f_{2} / f_{1}$, results in $f_{1}, f_{2}$-maps for DPOAEs. In these maps, fyequency-dependent patterns occur as a result of the relative maxima and minima in the DPOAEandiograms. The orientation of these patterns within the $f_{1}, f_{2}$-maps provide clues about the macromechanical tuning properties of the inner ear. As we described in chapter 1, the arising patterns in frog DPOAEs from the basilar papilla could be modeled by a simple, single nonlinear oscillator (the Duffing oscillator), thus providing additiond evidence that this papilla functions as a single auditory filter. But the patterns in DPOAEs from the amphibian papilla were similar to those observed in the BP, and not like the patterns found in DPOAEs from the mammalian cochlea. This suggests that the turing in the AP is different from that in the cochea, implying that the $\mathrm{AP}$ does not support mammalian-like traveling waves.

In chapter 2 the dependence of DPOAE amplitude and phase on stimulus levels is explored in both the AP, and the BP. Similar to other vertebrates, the increase in DPOAE-amplitude with increasing stimulus levels is not constant, but varies with absolute stimulus levels. As a result, the slope of DPOAE input/output-curves ( $1 / 0$-curves) is not constant. This variation in the slopes of $1 / 0$-curves can be explained by interference between two level-dependent emission components. Here, one component dominates for low-level stimuli, while the other dominates for highlevel stimuli. In conjunction with this two-component model, the DPOAE-phase is different between the low-level and the high-level region of the $1 / \mathrm{O}$-curve. We 
found a conspicuous difference in the low-level slopes of the 1/O-curves from the AP and the BP. While DPOAE-growth in the AP is compressive (similar to other vertebrates) it is expansive in the BP. This difference has led to the speculation that acoustical processing in the BP does not involve an active amplification mechanism.

An extension of the experiments from chapter 2 is presented in chapter 3. Here, we obtained data to characterize the level-dependence of DPOAEs for a range of different relative stimulus levels. The resulting $L_{1}, L_{2}$-maps support the idea of the two-component model for DPOAE-generation. We showed that the low lavel DPOAE-component could be modeled by a saturating nonlinearity, while the highlevel DPOAE-component was described by an expansive nonlinearity. This strongly suggests that in the frog ear each level-dependent component arises from a different nonlinear mechanism, contradicting recent objections against the applicability of the two-component model to mammalian DPOAEs. In addition, a clear difference was found between the low-level regions of mammalian and anuman $L_{11}, L_{2}$-maps. These differences do not seem to signify the involvement of different emission generationmechanisms in mammals and frogs. Rather, they may be explained, by differances in the level-dependence of (macromechanical) vibration-patterns of the different inner ear structures.

The effect of body-temperature on DPOAEs was investigated in chapter 4 . This was done to assess whether DPOAE-generation in the AP and the BP involves an active inner ear amplifier, respectively. The rationale for this is that the inmer ear amplifer requires energy. In biological systems, this energy must come from metabolic processes whose functionality depends on temperature. For sub-optimal temperatures metabolism is depressed, resulting in a decreased functioning of the inner eax amplifier. We found that the generation of DPOAEs from the BP was not affected by substantial cooling of the frog. At the same time, this cooling clearly did affect the generation of low-level DPOAEs from the AP. These findings provide strong ev idence that the BP indeed functions as a passive auditory receiver, a property that may be unique within vertebrate hearing. The absence of a temperature-serisitive inner ear amplifier may actually be beneficial to the frog. Given the poilkilothermic nature of anurans, their body-temperature will fuctwate considerably. With the passive basilar papilla, its spectral window will be independent of ambient and body-temperature: a consistent representation of the world is maintained.

In chapter 5 the DPOAE-recordings were supplemented with recordings made from nerve-fibers. Since the frequency-selectivity of the BP seems to arise from a mechanically-tuned mechanism, both neural- and DPOAE-tuning reflect the same filtering mechanism. Using the Duffing oscillator (sce chapter 1) as a model for the $B P$, several correlations between the tuning characteristics of nerve-fibers and DPOAEs are predicted. These predictions were tested by recording both DPOAE- 
andiograms and neural turing curves from the basilar papilla of frogs. These data exhibited similar correlations as were predicted by the simple model, supporting the idea that the anuran BP functions as a mechanically-tuned, single auditory filter. Nevertheless, the observed differences between model predictions and the obtained data indicate that the Duffing oscillator is "too simple" to represent the high-order tuning-mechanisms of the basilar papilla. 


\section{Detailed $f_{1}, f_{2}$ area study of distortion product otoacoustic emissions in the frog}

\section{Abstract}

Distortion product otoacoustic emissions (DPOAEs) are weak sounds emitted from the ear when it is stimulated with two tones. They are a manifestation of the nonlinear mechanics of the inner ear. As such, they provide a noninvasive tool for the study of the inner ear mechanics involved in the transduction of sound into nerve fiber activity. Based on the DPOAE phase behavior as a function of frequency, it is currently believed that mammalian DPOAEs are the combination of two components, each generated by a different mechanism located at a different location in the cochlea. In frogs, instead of a cochlea, two separate hearing papillae are present. Of these, the basilar papilla (BP) is a relatively simple structure that essentially functions as a single auditory filter. A two-mechanism model of DPOAE generation is not expected to apply to the BP. In contrast, the other hearing organ, the amphibIan papilla (AP), exhibits tonotopic organization. In the past, it has been suggested that this papilla supports a traveling wave in its tectorial membrane. Therefore, a two-mechanism model of DPOAE generation may be applicable for DPOAEs from the AP. In the present study we report on amplitude and phase of DPOAEs in the frog ear in a detailed $f_{1}, f_{2}$ area study. The result is markedly different from that in the mammalian cochlea. It indicates that DPOAEs generated by neither papilla agree with the two-mechanism traveling wave model. This confirms our expectation for the BP and does not support the hypothesized presence of a mechanical traveling wave in the AP. 


\subsection{Introduction}

Distortion product otodcoustic emissions (DPOAEs) are an acoustic phenomenon that can be observed in a healthy ear that is stimulated with two stimulus tones with properly chosen frequencies $\left(f_{1}, f_{2}\right.$, with $\left.f_{1}<f_{2}\right)$ and levels $\left(L_{1}\right.$ and $L_{2}$, re spectively). It is currently believed that in the mammalian cochlea, lower-sideband DPOAEs (with $f_{d y}<f_{1}, f_{2}$ ) are the result of two DPOAE components, each originating from a different location on the basilar membrane (Kim, 1980, Kemp and Brown, 1983, Brown et al, 1996, Talmadge et al., 1999, Shera and Guinar., 1999).

Shera and Guinan (1999) postulate that the fundamental distinction between these two components is not the different location but the different mechanism involved in their generation. Hence, they use the term two-rnechanism model of DPOAE generation. Here, nonlinear distortion generates the initial DPOAE com ponent (at the overlap region of the two stimulus tones) that may travel both in basal and apical directions in the cochlea. The apically-traveling DPOAE energy may be reflected due to local irregularities in the fine structure of the basilar membrane (around the characteristic frequency place of the distortion product) resulting in a second DPOAE component traveling to the base of the cochlea. The combination of the two basally-traweling components, together with multiple internal reflections (Stover et al, 1996), results in the DPOAE that can be recorded in the ear canal.

The frog ear is an interesting model for studying DPOAEs because, instead of a cochlea, it contains two distinct papillae that respond to airborne sound. The amphibian papilla (AP) consists of an elongated strip of sensory epithelium. It exhibits tonotopic organization (Lewis et al., 1982) and the presence of traveling waves in the tectorial membrane has been proposed (Lewis and Leverenz, 1983, Hillery and Narins, 1984, Lewis, 1984). Hair cells are innervated by both afferent and efferent nerve fibers, the former having tuming characteristics similar to those found in mamnals (Lewis, 1992). The AP may generate both spontaneous and distortion product OAEs (Van Dijk and Manley, 2001). The analogy of this papilla with the mammalian cochlea, te. the presence of tonotopic organization, suggests that a trwveling wave mechanism, with separate emission sources, may underlie DPOAE generation.

The basilar papilla (BP) is a much simpler structure. It consists of a small patch of sensory epithelium which is covered by a tectorial membrane (Lewis and Narins, 1999). In $R$. catesbeiana, hair cells in the BP are only innervated by afferent nerve fibers (Robbins et al., 1967, Frishkopf and Flock, 1974). In individual frogs, the vast majority of nerve fibers is tuned to a single frequency (Ronken, 1991, Van Dijk et al. 1997). Also, in ranid frogs the hair bundles are oriented in parallel (Lewis, 1978). These properties indicate that this papilla functions essentially as a single 
auditory filter (Ronken, 1990, Van Dijk and Manley, 2001) which makes it unlikely that DPOAE generation involves two different emission sources or a traveling wave mechanism.

Recently Knight and Kemp (2000) reported an $f_{1}, f_{2}$ area study of human DPOAEs. They showed that patterns in the amplitude and phase data, when represented in an $f_{2} / f_{1}$ versus $f_{d p}$ plot, are oriented either horizontally or vertically. The pattems are consistent with the two-mechanism model of DPOAE generation. A similar method to analyze the phase data was presented by Schneider et al. (2003). Although these methods offer no technique to study the two components separately, they do provide a way to study which component dominates the recorded DPOAE.

In the present study we report an $f_{1}, f_{2}$ area study of amplitude and phase of DPOAEs in the leopard frog, $R$. pipiens pipiens. The patterns observed deviate considerably from those observed in mammals. We conclude that a cochlear-wike, two-source DPOAE model does not apply to the amphibian inner ear. This confirms our expectations for the BP, but does not support the hypothesized presence of a mechanical traveling wave in the AP.

\subsection{Methods}

Distortion product otoacoustic emissions were recorded from Northern leopard frogs (Rana pipiens pipiens): $\mathrm{n}=5$ females; body mass: $20.8-31.9 \mathrm{~g}$ (mean: $27.2 \mathrm{~g}$ ), snout-vent length: $7.11-7.84 \mathrm{~cm}$ (mean: $7.42 \mathrm{~cm}$ ). Animals were anesthetized with an intramuscular injection of pentobarbital sodium solution (Nembutal: $60 \mathrm{mg} / \mathrm{ml}$; $1.0 \mu \mathrm{l} / \mathrm{g}$ body mass) in one of the hindlimbs. Measurements were performed in a sound-attenuating chamber, with the frog placed on a vibration isolation table. During the experiments, the animal was covered by gauze soaked in tap water to prevent dehydration and to facilitate cutaneous respiration. In oach subject the left ear was tested.

DPOAEs were recorded with a probe assembly that contained two miniature transducers (ER-10C; Etymotic Research) for stimulus generation, and one $\frac{1}{2}$ " condenser microphone (Brüel \& Kjær type 4134) for emission recording. The open end of the probe assembly was carefully placed against the skin surrounding the frog's tympanic membrane. A tight seal between probe and skin was obtained using silicone grease.

DPOAEs were evoked by two stimulus tones, with frequencies $f_{1}$ and $f_{2}$ (where $f_{2} / f_{1}>1$ ) and levels $L_{1}$ and $L_{2}$, each of which was played from a separate miniature speaker. The stimulus tones were generated from two separate D/A channels (RP2: Tucker Davis Technologies, Gainesville, FL, USA) and the level of each tone was adjusted with a separate programmable attenuator (PA5: Tucker Davis Tech- 
nologies). The microphone signal was amplified $60 \mathrm{~dB}$ with a pre-amplifier (Brïel \& Kjar type 2609) and recorded on computer dise using an A/D converter (RP2. Tucker Davis Technologies). During the experiments, the amplified microphone signal was also fed into a spectrum analyzer (Stanford Research Systems SR770) in order to monitor the DPOAEs online. Customized software, written in MATLAB (The MathWorks, Inc) and RPvds (Tucker Davis Technologies) were used to control stimulus tone generation and signal recording.

For each frog, recordings were made in a series of fixed-frequency ratio (fixed$\left.f_{2} / f_{1}\right)$ sweeps from low to high $f_{1}$, starting with the lowest frequency ratio $f_{2} / f_{1}=$ 1.02 and ending with a ratio of 1.70 . In each sweep, $f_{1}$ varied from 213 to $2774 \mathrm{~Hz}$ in approximately $30 \mathrm{~Hz}$ steps. The levels of the stimulus tones, $L_{1}=L_{2}=76 \mathrm{~dB}$ SPL, were kept constant for all stimulus frequency combinations. These relatively large stimulus amplitudes were used in order to evoke DPOAEs over a wide enough stimulus frequency $\left(f_{1}, f_{2} / f_{1}\right)$ area to reliably unwrap the phase data. At the start and at regular intervals during the experiment a calibration procedure was performed to set the levels of the stimulus tones. In addition, the condition and the position of the animal was checked, and the gauze covering the animal re-moisturized. To determine the nonlinear distortion of the DPOAE recording setup, experiments were performed with the open end of the probe pressed against a solid surface. This resulted in no detectable system distortion for any of the stimulus conditions used.

At each frequency pair $\left(f_{1}, f_{2}\right)$, stimulus tones were played continuously for 50,000 sample points $(\approx 4.10 \mathrm{sec}$ with a sample frequency of $12.2 \mathrm{kHz}$ ). The frequency $f_{1}$ was chosen such that 100 periods of this stimulus tone matched exactly an integer number of sample points. By performing the experiments in the form of fixed-ratio sweeps, where the ratio $f_{2} / f_{1}$ was chosen with a maximum of two significant digits (e.g. $f_{2} / f_{1}=1.04$ or $f_{2} / f_{1}=1.30$ ), all other frequencies of interest (i.e. stimulus frequency $f_{2}$ and all DPOAEs) were also exactly periodic over the same integer number of sample points. This method has two advantages in the analysis of the recorded signal. First, the total recorded signal (50,000 sample points) can be divided into a series of blocks that all have the same starting phase of the stimulus tones and DPOAEs. These blocks can be averaged in order to reduce the noise floor, without affecting the amplitude and phase of the tones of interest. Secondly, the exact periodicity of all tones of interest ensures that in the Fourier analysis the corresponding frequencies each fall exactly in the center of a frequency bin. This abolishes spectral smearing, and provides an accurate estimate of the amplitudes and phases of the DPOAEs.

In the analysis of the digitized microphone signal the first periodic block was omitted to exclude onset phenomena. The remaining signal was used to calculate the amplitudes $\left(L_{1}, L_{2}\right)$ and phases $\left(\phi_{f_{1}}, \phi_{f_{2}}\right)$ of the stimulus tones using Fourier 
analysis. The parameters thus obtained were nsed to subtract the stimulus tones from the microphone signal in the time domain. For the remaining signal, containing only the DPOAEs and system noise, each periodic block was subjected to a level-crossing artifact rejection method. Artifact-free blocks were sub-averaged in two buffers $A$ and $B$. The awerage $(A+B) / 2$ was used to estimate the DPOAEs amplitudes and phases, whereas the diflerence A-B provided an estimate for the noise levels. As with the stimulus tones, amplitudes and phases of the DPOAEs were calculated using Foumer analysis.

The "Principles of Animal Care" (NIH publication 85-23, revised 1985) and USA regulations were followed throughout this study, and protocols were approved by the University of Califormia Animal Research Committee.

\subsubsection{Data analysis and representation}

For each clistortion product, amplitude and phase data were arranged in an $\left(f_{1}, / / f f_{1}\right)$ area matrix. Only data points for which the emission amplitude exceeded the noise: floor by $6 \mathrm{~dB}$ were included in the matrices. Phase data are represented relative to the phase of the stimulus tones. That is, for the distortion product at $f_{d p}=(n+1) f_{1}-n f_{2}$, the relative phase is: $\Phi_{f_{d n}}=\phi_{f_{d p}}-(n+1) \phi_{f_{1}}+n \phi_{f_{2}}$. Subsequently, the phase data was unwrapped in two dimensions (constant- $f_{1}$ and constant- $\left.f_{2} / f_{1}\right)$ by removing $2 \pi$ discontinuities.

Phase slope delay (group delay) is defined as:

$$
D=-\frac{1}{2 \pi} \frac{d \Phi_{d p}}{d d^{f} d p}
$$

whene $\Phi_{d p}$ is the relative phase of the distortion product, and is considered as a function of $f_{1}$ for a constant ratio $f_{2} / f_{1}$. The slope d $\Phi / d f$ of the phase was calculated by fitting a straight line to three points, $f_{1}$ and the two neighboring points. This was only done when all three points had a DPOAE arnplitude exceeding the noise floor by $6 \mathrm{~dB}$. To prevent contamination of the results, only slopes with a correlation coefficient exceeding 0.988 (corresponding to $p=0.10$ ) are considered in this paper.

\section{$1.3 \quad$ Results}

Distortion product otoacoustic emissions could be detected in each individual frog investigated. We systematically analyzed the recorded data for distortion products at frequencies $2 f_{1}-f_{2}, 2 f_{2}-f_{1}, 3 f_{1}-2 f_{2}$ and $3 f_{2}-2 f_{1}$.

The dependence of DPOAE amplitude on the stimulus frequencies follows a complicated pattern. Some of these patterns are illustrated by the contourlines in. Fig. 1.1. In each panel of Fig. 1.1, the bottom half refers to the DPOAE at $2 f_{2}-f_{1}$, 

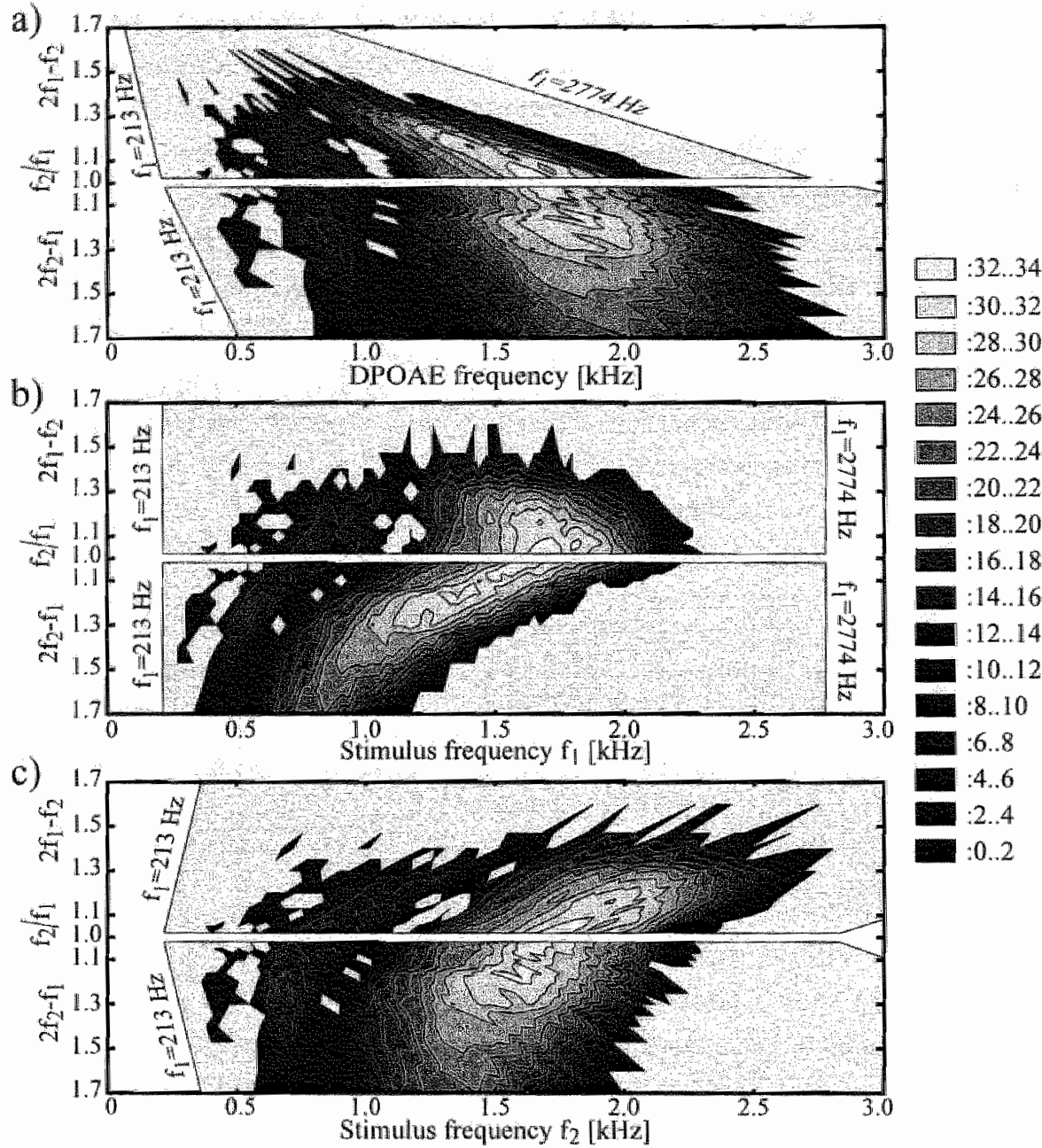

Figure 1.1: Amplitude at $2 f_{1}-f_{2}$ and $2 f_{2}-f_{1}$ in dB SPL, evoked with $L_{1}=L_{2}=76$ AB SPL, recorded in one frog. The different panels represent the same data set with $f_{2} / f_{1}$ versus either (a) distortion product frequency, (b) stimulus frequency $f_{3}$ or (c) stimulus frequency $f_{3}$. In each panel, the same gray-coding is used. Individual contourlines are drawn at $2 \mathrm{~dB}$ intervals. The shaded area represents the $\left(f_{1}, f_{2}\right)$ area studhed. For DPOAEs at $2 f_{1}-f_{2}$, amplitude peaks and valleys result in patserms that are vertical when data are plotted as function of stimulus frequency $f_{1}$ (panel $b$, upper half). In contrast, for DPOAE at $2 f_{2}-f_{1}$ a similar vertical pattern occurs when the data are plotted as a function of either stimulus frequency $f_{2}$ (panel $c$, lower half) or DPOAE frequency (panel $a$, lower half). Note that two broad frequency regions can be seen where emission amplitudes show a relative maximum. These two regions are separated by a notch region centered around $f_{1}=1250 \mathrm{~Hz}\left(\right.$ for $\left.2 f_{1}-f_{2}\right)$ or $f_{d p}$ slightly below $1250 \mathrm{~Hz}\left(\right.$ for $\left.2 f_{2}-f_{1}\right)$ where emissions are considerable lower in amplitude, or even undetectable. This frequency of $1250 \mathrm{~Hz}$ corresponds with the separation in characteristic frequency ranges of nerve fibers from the amplabian and basilar papilla (Ronken, 1991). 
and the top half illustrates DPOAE at $2 f_{1}-f_{2}$. In general, DPOAE amplitudes were relatively large in two broad frequency ranges. Separating these two peak regions is at well-defined valley where DPOAE amplitudes dropped considerably. As is obvious from the figure, the orientation of these DPOAE peak and valley regions depends on the parameter plotted on the abscissa. When the DPOAE at $2 f_{1}-f_{2}$ is plotted as a function of stimulus frequency $f_{1}$ (Fig. $1.1 \mathrm{~b}$, upper thalf) these DPOAE amplitude peaks and valleys give rise to a vertical pattern in the contourlines. In contrast, when the amplitude of this DPOAE is plotted as a function of either DPOAE frequency (Fig. 1.1a, upper half) or stimulus frequency $f_{2}$ (Fig: 1.1c, upper half) a diagonal pattern in the contourlines appears.

From inspection of the lower halves of the panels in Fig. 1.1 it is clear that, for the DPOAE at $2 f_{2}-f_{1}$ the situation is similar, but slightly more complex. Here, the peaks and valley in DPOAE amplitude result in an approximately vertical pattern in the contour plots when either the DPOAE frequency (Fig. 1.1a) or the stimulus frequency $f_{2}$ (Fig. 1.1c) is on the abscissa. With the stimulus frequency $f_{1}$ on the horizontal axis (Fig. 1.1b) the pattern in the contour plot, is diagonal,

DPOAEs at $2 f_{2}-f_{1}$ could be detected for much wider stimulus frequency ratios than those at $2 f_{1}-f_{2}$. For example, at the largest ratio studied $\left(f_{2} / f_{1}=1.7\right.$, i.e. the horizontal boundaries of the panels) DPOAEs at $2 f_{2}-f_{1}$ were still as large as $26 \mathrm{~dB}$ SPL, while for the same ratio DPOAEs at $2 f_{1}-f_{2}$ were indistinguishable from the noise floor.

DPOAE-audiograms recorded in the frog typically show a bimodal dependence on frequency (Van Dijk and Manley, 2001, Van Dijk et al., 2003, Meenderink and Van Dijk, 2004). They exhibit two frequency regions with elevated DPOAE amplitudes which are separated by a clear notch in DPOAE amplitude. The area representations in Fig. 1.1 show that this bimodal dependence is observed irrespective of the stimulus frequency ratio $f_{2} / f_{1}$ used. At the same time these representations show that whether the notch shifts in frequency for DPOAE-audiograms recorded with different frequency ratios $f_{2} / f_{1}$ depends on the parameter plotted on the x-axis. For DPOAEs at $2 f_{1}-f_{2}$, the notch in DPOAE-audiograms does not shift position when stimulus frequency $f_{1}$ is on the $\mathrm{x}$-axis, it is centered around approximately $f_{1}=1250 \mathrm{~Hz}$. For DPOAEs at $2 f_{2}-f_{1}$ the notch is observed at a fixed frequency slightly below $f_{d y}=1250 \mathrm{~Hz}$ when distortion product frequency is on the abscissa.

Compared to the amplitude, the pattern observed in the DPOAE phase is sinpler. Fig. 1.2 illustrates the phase data accompanying the amplitude data presented in Fig. 1.1. Again, the bottom half of the panel illustrates DPOAEs at $2 f_{2}-f_{1}$, and the top half refers to DPOAEs at $2 f_{1}-f_{2}$. For DPOAEs at both $2 f_{1}-f_{2}$ and $2 f_{2}-f_{1}$, contourlines, i.e. lines of equal relative phase of the DPOAEs, are (nearly) vertical when plotted as a function of DPOAE frequency (Fig. 1.2). These vertical 


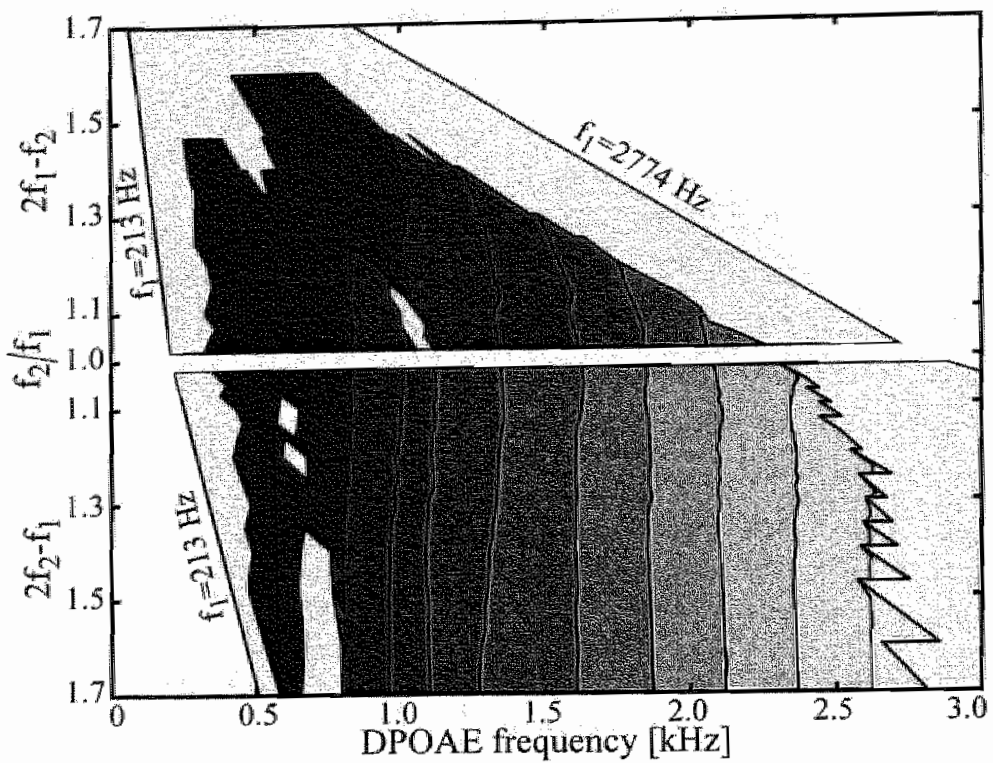

Figure 1.2: Phase data (in rad) for $2 f_{1}-f_{2}$ and $2 f_{2}-f_{1}$ plotted as a function of distortion product frequency. The phase data conrespond to the amplitude data represented in Fig. 1.1. Contourlines are drawn at $\pi$ rad intervals. The shaded area represents the $\left(f_{1}, f_{2}\right)$ area studied. The vertical contourlines indicate that the relative phase of the DPOAEs depends on the frequency of the distortion product itself, and is independent of both the absolute and relative frequencies of the two stimulus tones.

contourlines indicate that DPOAE phase primarily depends on DPOAE frequency, and is nearly independent of the stimulus frequency ratio $f_{2} / f_{1}$.

The distance between the contourlines in the phase data showed a bimodal dependence on DPOAE frequency, similar to the bimodal dependence observed in the DPOAE amplitude. This is reflected in the group dedays of the DPOAEs. Fig. 1.3a shows the combined group delays (average \pm S.D. of all frogs and all frequency ratios) for DPOAEs at $2 f_{1}-f_{2}$ plotted as function of stimulus frequency $f_{11}$. For $f_{1}<1250$ $\mathrm{H} z$, the group delays were larger (corresponding to closer spaced contourlines in Fig. 1.2) compared to the group delays for DPOAEs recorded with $f_{1}>1250 \mathrm{~Hz}$. A similar plot is given for DPOAEs at $2 f_{2}-f_{1}$ in Fig. $1.3 \mathrm{~b}$. Here, the group delays are relative constant for $f_{1}>800 \mathrm{~Hz}$. Below $800 \mathrm{~Hz}$, the group delays decrease with increasing stimulus frequency.

The presence of DPOAEs was not restricted to the frequencies $2 f_{1}-f_{2}$ and $2 f_{2}-f_{1}$. In all frogs studied DPOAEs were also detectable at $3 f_{1}-2 f_{2}$ and $3 f_{2}-2 f_{1}$. However, the frequency areas in which these DPOAEs could be observed were restricted to both smaller relative $\left(i_{2}\right.$. $f_{2} / f_{1}$ ) and absolute stimulus frequency ranges. 

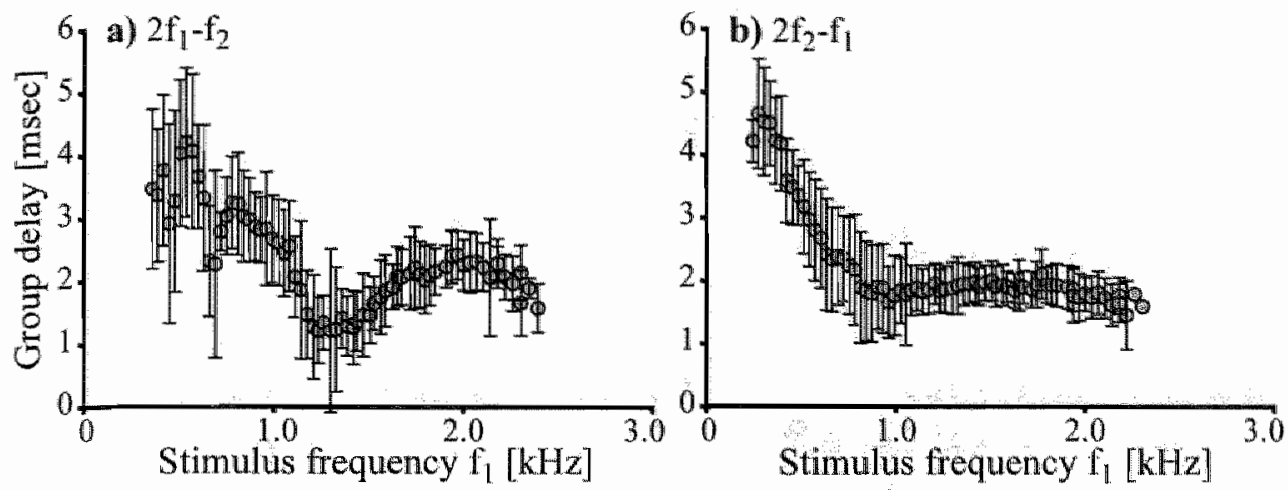

Figure 1.3: Group delays calculated for a fixed-ratio recording paradigm plotted as a function of stimulus frequency $f_{3}$. The two panels represent group delays for (a) $2 f_{1}-f_{2}$, and (b) $2 f_{2}-f_{1}$. Each point gives the combined average over all frogs and all $f_{2} / f_{1}$ ratios. The error bars denote the standard deviation ( \pm 1 S.D.). Points lacking an error bar represent single obserwations (i.e. one frog and one $f_{2} / f_{1}$ ratio).

This was due to the smaller amplitudes of these DPOAEs. Fig. 1.4 shows amplitudes of DPOAE at $3 f_{1}-2 f_{2}$ and $3 f_{2}-2 f_{1}$ in a similar representation as Fig. 1.1. For DPOAE amplitude at $3 f_{1}-2 f_{2}$, i.e. the upper halves of Fig. 1.4 , the pealss and valleys do not shift in frequency with varying stimulus frequency ratio when stimulus frequency $f_{1}$ (Fig. $1.4 \mathrm{~b}$ ) is on the abscissa. In contrast, the lower halves of Fig. 1.4, i.e. DPOAE at $3 f_{2}-2 f_{1}$, the amplitude peaks and valleys give rise to an approximately vertical pattern in the contour plots when either DPOAE frequency (Fig. 1.4a) or stimulus frequency $f_{2}$ (Fig. 1.4c) is on the horizontal axis.

In only one frog were these higher-order DPOAEs observed over a large enough stimulus frequency area to reliably unwap the phase data. The phase data obtained in this frog are shown in Fig. 1.5.

This figure corresponds to the amplitude data shown in Fig. 1.4. For both of the higher-order DPOAEs, phase showed vertical contourlines when the DPOAE frequency is plotted on the horizontal axis (Fig. 1.5). These observations in both the amplitude and phase data for DPOAE at $3 f_{1}-2 f_{2}$ and $3 f_{2}-2 f_{1}$ are sirnilar to those made for DPOAE at $2 f_{1}-f_{2}$ and $2 f_{2}-f_{1}$.

\subsection{Discussion}

In the present study, DPOAEs were only found over a limited range of stimulus frequencies. This range is within the frequency range to which nerve fibers from the frog ear are tuned (100 to $2300 \mathrm{~Hz}$ in R. pipiens pipiens; Ronken, 1990). This frequency 

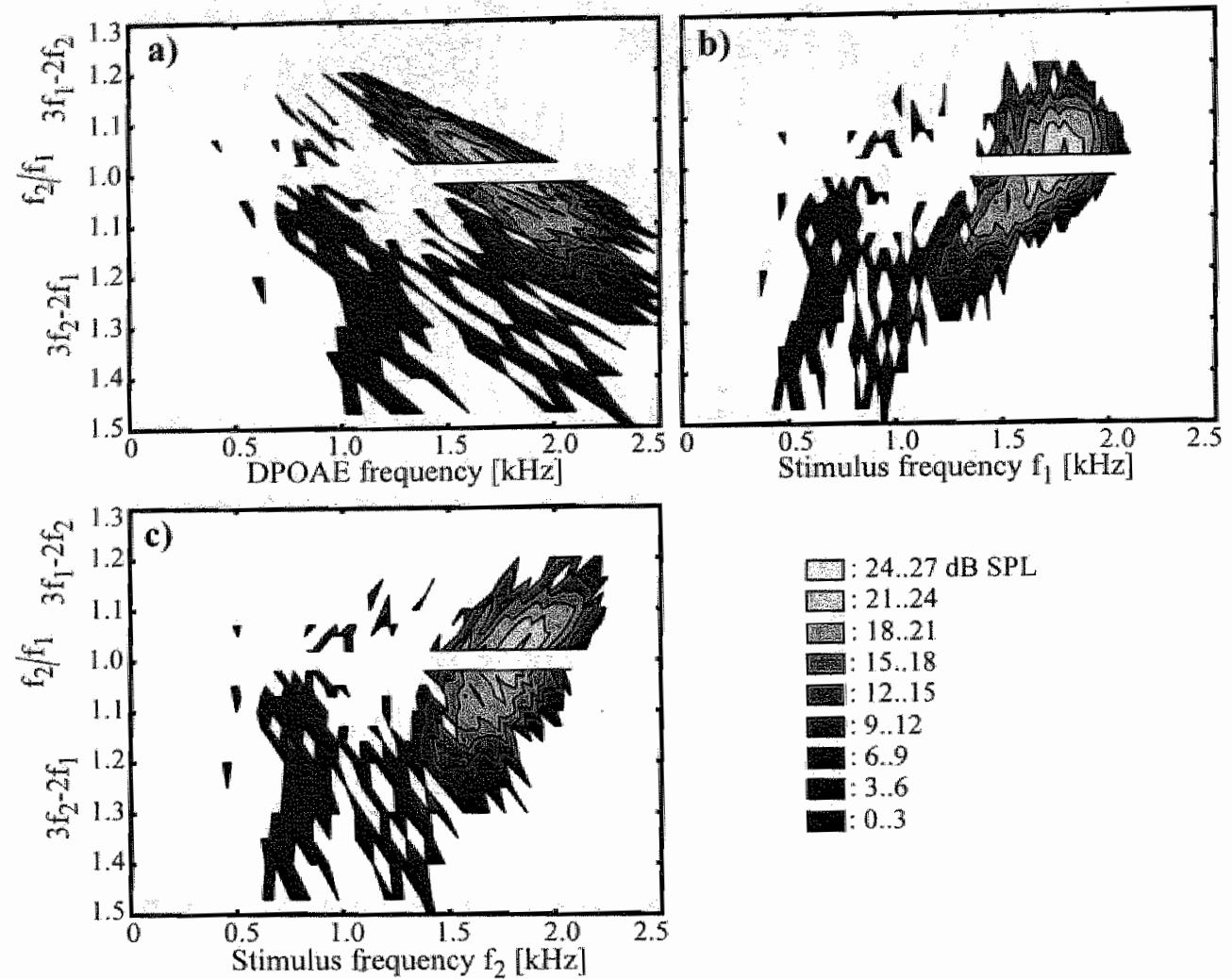

Figure 1.4: Amplitude and phase of DPOAEs at $3 f_{1}-2 f_{2}$ and $3 f_{2}-2 f_{1}$. The same amplitwde data (dB SPL) are platted with $f_{2} / f_{1}$ wersus either (a) distortion product frequency, (b) stimulus frequency $f_{1}$ or $(c)$ stimulus frequency $f_{2}$. In each panel, the same gray-coding is used as given in the key. For clarity, individual contourlines are drawn at, $3 \mathrm{~dB}$ intervals rather than at $2 \mathrm{~dB}$ intervals as was used in Fig. 1.1. For DPOAEs at $3 f_{1}-2 f_{2}$ a vertical pattern occurs when plotted as a function of $f_{1}$ (panel b). In contrast, for DPOAEs at $3 f_{2}-2 f_{1}$ patterms are only close to wertical when either stinulus frequency $f_{2}$ (panel $c$ ) or DPOAE frequency (panel a) is on the horizontal axis. Although the amplitudes are reduced, these results are qualitatively the same as those obserwed for DPOAEs at $2 f_{1}-f_{2}$ and $2 f_{2}-f(F i g .1 .1)$. 


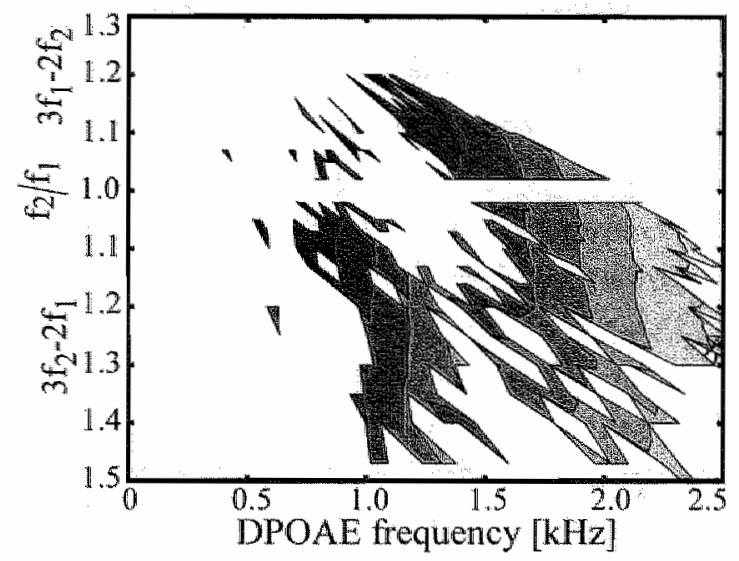

Figure 1.5: Relative phase of DPOAE at $3 f_{1}-2 f_{2}$ and $3 f_{2}-2 f_{1}$ plotted as a function of distortion product frequency. Data correspond to amplitude data represented in Fig. 1.4. Contourlines are drawn at $\pi \mathrm{rad}$ intervals. The vertical contourlines indicate that the relative phase of the DPOAEs depends on the frequency of the distortion product alone, and is independent of both the absolute and relative frequencies of the two stimulus tones. This dependence on distortion product frequency is similar as to that observed for the relative phase data for DPOAEs at $2 f_{1}-f_{2}$ and $2 f_{2}-f_{1}$ (Fig. 1.2).

range could be divided in two broad frequency sub-regions where DPOAEs exhibited relatively large amplitudes. These sub-regions were separated by a frequency notch where relatively small DPOAE amplitudes were found. Based on the bandwidth of neural tuning curves recorded in $R$. pipiens pipiens, Ronken (1991) estimated that nerve fibers tuned to frequencies less than $1250 \mathrm{~Hz}$ innervate the amphibian papilla, while nerve fibers tmed to higher frequencies innervate the basilar papilla. The correspondence between the DPOAE data and the nerve fiber data suggests that frog DPOAEs originate from both of the papillae present in the inner ear. A similar observation was made by Van Dijk and Manley (2001), Van Dijk et al. (2003) and Meenderink and Vari Dijk (2004). At the stimulus levels used here $\left(L_{1}=L_{2}=76\right.$ AB SPL), DPOAE amplitude was larger in the BP than in the AP for all frogs.

The phase, obtained when recording these DPOAE-audiograms, varies with varying stimulus requencies. For DPOAEs from the AP, the rate of plase change decreases for increasing frequency, resulting in the observed frequency dependence of the group delay (Fig. 1.3). For DPOAEs generated in the BP, growp delays are relaw tively constant, signifying a nearly linear change in phase with changing frequencies. This dependence of group delay on frequency (in the AP) is not only similar to the frequency dependence of response delays reported for neural data in frogs (Hillery and Narins, 1984, 1987), but also to neural delays reported for all vertebrate classes 
(see Manley et al, 1990 for an overview). In mammals, these delays have been attributed to the delay time of the traveling wave on the basilar membrane. In fact, the close correspondence between mammalian delays and those found in the coqui frog (Hillery and Narins, 1984) led to the hypothesis of a traveling wave in the tectorial membrane that covers the frog amphibian papilla. However, in the bobtail lizard no traveling wave on the basilar membrane is present (Manley et al., 1988), while the neural delays again show at similar dependence on frequency (Manley et al, 1990). It seems that the relatively similar neural delay patterns found across non-mammalian vertebrate classes arise from similarly-tuned filter arrays. Thus, despite the very different auxiliary structures in the inner ears of the different vertebrate classes, filter mechanisms may be similar across species.

The patterns in DPOAE amplitude and phase described here deviate considerably from those reported for mammals (Knight and Kemp, 2000, Schneider et al., 2003). In the mammalian cochlea, the patterns observed in the amplitude and accompanying phase data are oriented parallel to each other: when plotted in an $f_{2} / f_{1}$ versus $f_{d p}$ area map, both amplitude and phase display either a vertical or a horizontal orientation. A vertical orientation of the patterns occurs when the recorded DPOAE is dominated by a reflection component. This component is thought to arise via a mechanism of linear reflection occuring at irregularities of the cochlea around the characteristic place of the DPOAE (Shera and Guinan, 1999). Consequently, DPOAE amplitude and phase primarily depends on the distortion product frequency, trast, a horizontal orientation of the patterns in amplitude and accompanying phase data is found if not the reflection component but rather the nonlinear distortion component dominates the recorded DPOAE signal. This component is thought to arise via nonlinear distortion at that region along the basilar membrane where the response envelopes of the two stimulus tones overlap maximally, i.e. around the with respect to frequency, the frequency $f_{2}$. By assuming a scale-invariant cochlea on the ratio $f_{2} / f_{1}$ and is independent of horizontal orientation of the patten in af DPAE frequency, resulting in the observed

The close link between the patterns amplitude and phase. in the mammalian cochlea the patterns in DPOAE amplitude and phase, as seen for lower sideband DPOAEs (with present in the frog. This is seen most clearly $3 f_{1}-2 f_{2}$. Here, the relative phase of $f_{d p}<f_{1}, f_{2}$ ), i.e. DPOAEs at $2 f_{1}-f_{2}$ and the DPOAE frequency, i.e. vertica of the DPOAEs shows a dependence on only and 1.5. However, in the corresponding area in the upper halves of Figs. 1.2 patterns are clearly diagonal (Figs. 1.1 and maps of the amplitude the observed orientation of the patterns in amplitude and 1.4a, upper halves). This diagonal an $f_{2} / f_{1}$ versus 
$f_{d p}$ map indicates that the DPOAE amplitude does not exclusively depend on either the relative stimulus frequencies or the DPOAE frequency. Rather, the observed patterns are oriented vertically when the stimulus frequency $f_{1}$ is plotted on the horizontal axis (Figs. 1.1b and 1.4b, upper halves).

For upper sideband DPOAE (with $f_{d p}>f_{1}, f_{2}$ ), i.e. $2 f_{2}-f_{1}$ and $3 f_{2}-2 f_{1}$, similar differences between mammalian and frog DPOAEs were present. Again, the vertical contourlines in the phase data (Figs. 1.2 and 1.5, lower halves) indicate a dependence of DPOAE phase on distortion product frequency alone. On the other hand, the patterns in amplitude data are slightly diagonal when DPOAE frequency is on the horizontal axis. A similar diagonal orientation is observed when amplitude is plotted as function of stimulus frequency $f_{2}$ (Figs. $1.1 \mathrm{c}$ and $1.4 \mathrm{c}$, lower halves).

Knight and Kemp (2001) describe their results, obtained in the cochlea, with a transmission line model. The model is not a physical description of the cochlea, but it incorporates some generic cochlear properties, such as the traveling wave on the basilar membrane. The difference in the amplitude and phase patterns observed in mammals and frogs indicate that the model does not apply to the frog inner ear. In other words, our results do not provide any evidence for cochlear-like traveling wave mechanics in the frog inner ear.

But if no cochlear-like traveling wave mechanics are involved in the sound transduction in the frog inner ear, what kind of mechanism is?

Of the two hearing organs present in the frog inner ear, the basilar papilla is the simplest in structure and function. It consists of a small patch of hair cells, which is embedded in a solid surface and covered by a tectorial membrane. The papilla. essentially functions as a single auditory filter. Evidence for this is provided by tuning curves obtained from nerve fibers innervating this organ: in individual frogs almost all nerve fibers are tuned to a single frequency, with the shapes of all tuning curves being remarkably similar (Ronken, 1990). Obviously, a traveling wave model is not applicable here.

Based on the notion that the BP functions as a single auditory filter, we will consider a single resonator, the Duffing oscillator, as a simple model for DPOAE generation in this papilla (see also Van Dijk and Manley, 2001). The Duffing oscillator is described by the nonlinear second-order equation

$$
m \ddot{x}+R \dot{x}+k(x) x=F(t)
$$

where $m$ is a mass, whose movement is driven by an external force $F(t)$. The linear resistance $R$ impedes this movement, while a nonlinear stiffness $k(x)=k_{0}\left(1+\frac{\frac{\pi}{2}^{2}}{x_{t}^{2}}\right)$ tries to restore the mass' position $x$ to its equilibrium position $x=0$.

When the oscillatior is driven by a two-tone force

$$
F(t)=A_{1} \sin \left(2 \pi f_{1} t\right)+A_{2} \sin \left(2 \pi f_{2} t\right)
$$




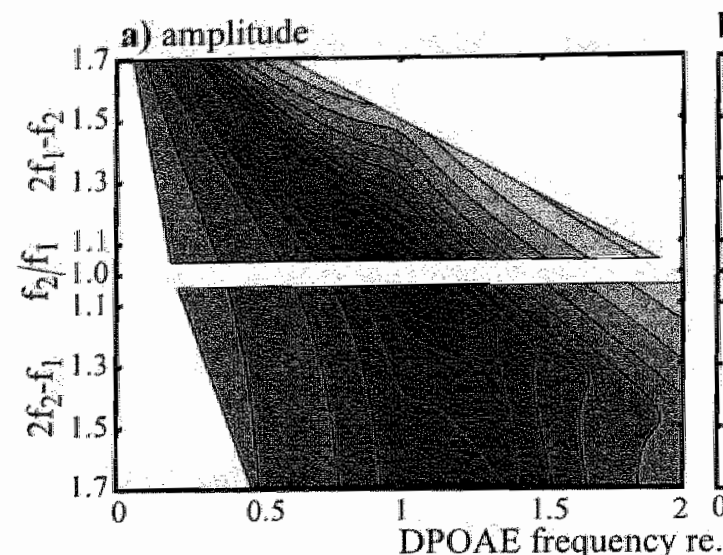

b) phase

Figure 1.6: Intermodulation products at $2 f_{1}-f_{2}$ and $2 f_{2}-f_{1}$ obtained from a single nonlinear resonator used to model the frog basilar papilla. The model consisted of a Duffing oscillator which was driven by a two-tone force. (a) Amplitude in dB plotted as a function of intermodulation product frequency, similar as the presentation of DPOAE amplitude in Fig. 1.1a. Individual contourlines are drawn at $10 \mathrm{~dB}$ intervals. The pattern formed by the contourlines is very similat to that observed in the recorded DPOAE. For $2 f_{2}-f_{2}$ (upper half of the panel) the pattern is oriented diagonally, following lines of fixed- $f$. In contrast, for $2 f_{2}-f_{1}$ (lower half of the panel) the pattern is closer to vertical. The individual contourlines for both intermodulation products all exthibit three more or less distinct lobes, which are not observed in the DPOAE data. These lobes occur whenever $f_{1}, f_{2}$ or $f_{d p}$ comcides with the resonance frequency of the oscillator. Apart from these lobes, the pattern of the contourlines displayed here is similar to that in Fig. 1.la for the basilar papilla ( $>1250 \mathrm{~Hz}$ ). (b) Phase, in rad, accompanying the amplitude data in 1.6 a. It is plotted relative to the $f_{1}$ and $f_{2}$ stimulus frequency components in the response $x(t)$ (See Eq. 1.2), rather than the force $F(t)$. This compensates for the nonlinear phase response of the model. Individual contourlines are drawn at 0.1 * $\pi$ rad intervals. Again, the data are plotted as a function of intermodulation product frequency. As for the DPOAE phase (lig. 1.2), the contourlines are vertical, irrespective of the intermodulation frequency (i.e. $2 f_{1}-f_{2}$ or $\left.2 f_{2}-f_{1}\right)$. This indicates that the phase primarily depends on the frequency of the intermodulation product, and not on the two stimulus frequencies.

cubic distortion products are present in the response $x(t)$. We simulated the oscillator using a range of absolute and relative stimulus frequencies: $f_{11}$ varied between 0.2 and 2.0 in 0.01 steps, with the resonance frequency of the oscillator being 1 , while $f_{2} / f_{1}$ was between 1.04 and 1.7 in steps of 0.01 . This allowed for a qualitative comparison between the model and the DPOAEs recorded in the frog. The results of the model are shown in Fig. 1.6 for $m=1, r=1, k_{0}=4 k^{2} \pi^{2}, x_{0}=1$, and $A_{1}=A_{2}=1$. In this figme, the amplitude and phase data are plotted as a function of intermodulation product frequency, similar to what was done for the DPOAEs 
recorded from the frog ear (Figs. 1.1a and 1.2).

The amplitude contourlines, drawn at $10 \mathrm{~dB}$ intervals (Fig. 1.6a), show a pattern that is very similar to that observed in the recorded DPOAEs (Fig. 1.1a). For intermodulation products at $2 f_{1}-f_{2}$ (Fig. 1.6a, upper half) this pattern has a diagonal orientation, following lines of fixed- $f_{1}$. In contrast, for intermodulation products at $2 f_{2}-f_{1}$ (Fig. 1.6a, lower half) the pattern in the contourlines is approximately vertical. Notice that in both the lower and upper half of the panel contourlines exhibit three more or less pronounced lobes. These arise whenever $f_{1}, f_{2}$ or $f_{d y}$ coincides with the resonance frequency of the oscillator.

The phase of the intermodulation products produced by the model are shown in Fig. 1.6b. It can be seen that the phase obtained with a single fixed-ratio sweep remains largely unchanged. Only when the intermodulation product frequency varies from slightly below to slightly above the resonance frequency, does phase change rapidly by $\pi$ rad. The contourlines in Fig. $1.6 \mathrm{~b}$ follow lines of equal intermodulation product frequency, resulting in a vertically-oriented pattern which is similar to that observed in frog DPOAE.

The qualitative good agreement between the patterns in DPOAE and intermodulation products, both in amplitude and phase, provides further support that the frog basilar papilla functions as a single (nonlinear) resonator.

DPOAEs from the amphibian papilla, the other hearing organ in the frog ear, behave similar to those from the $\mathrm{BP}$ : the patterns of DPOAE amplitude and phase (Figs. 1.1 and 1.2, respectively) are similar below (AP) and above (BP) the notch frequency of $1250 \mathrm{~Hz}$. Consequently, as the BP, emissions from the AP can be described by the simple oscillator model given in Eq. 1.2. However, the AP is not a single auditory filter, but functions as a tonotopically-organized array of auditory filters (Lewis and Leverenz, 1983). How can the simple BP-like DPOAE characteristics be reconciled with the structural and functional properties of the AP? We will discuss various factors which may contribute to the answer of this question, but at present we are unable to provide a definitive answer.

The AP is situated in a short chamber which runs from the periotic canal to the saccular recess. A contact membrane separates the perilymph in the periotic canal from the endolymph in the papillar space. The sensory hair cells are situated in the roof of the chamber, and are embedded in a rigid support structure. Hanging from the hair cells is an acellular structure, the tectorial membrane. Approximately half-way along the papilla, a tectorial curtain projects from the tectorial membrane to the floor of the papillar chamber. Due to its positioning within the chamber, any fluid movement along the principal axis of the papilla will result in movement of this tectorial curtain.

By tracing auditory nerve fibers with known characteristic frequencies to their 
point of innervation within the $A P$, Lewis et al. (1982) showed that the tonotopic organzation als along the principal axis of the papila. Here, the highest frequencies are at the candal part of the AP (close to the contact membrane that separates the peif-and endolymph), while towands the rostral end (closer to the sacculus) the aharacteristic frequency of nerve fbers decreases. Although acoustic energy enters the papillar chamber from the sacular space, (Purgue and Naxins, 2000a), it seems that the excitation moves from high to low frequencies, similar to the cochlea. This is mantest in the first-spike latency for auditory nerve fibers, which is longest for low-frequency fibers and shortest for fbers suned to the highest frequencies within the AP (Hillery and Narins, 1987 ). Neural tuning curves from the AP, show shallow slopes below the characteristic frequency, and steep slopes above (Narins and Hillery, 1983). Therefore, Lewis and Leverenz (1983) concluded that the AP, like the cochlea, may be modelled as a transmission-line low-pass fiter: each section of the papilla absorbs high-frequency acoustic energy, but passes lower frequencies to the subsequent sections in the structure. Although such a model describes the functional behavior of the papilla, Lewis and Leverenz (1983) were unable to reconcile it with the known anatomical gradients in the papilla.

They enphasized that the implementation of such bow-pass filter mechanism in the amphibian papilla must be very different from that in the cochlea. The basilar membrane in the cochlea is between two fuid-filled scalae, and is excited by a pressure difference between these scalae. The mechanical coupling between the different sections of the basilar membrane is provided through the fluid within the scalae: when the basilar membrane moves down at a particular location it pushes away fluid within the scala tympani. This fluid has to push up (towards scala vestibuli) the basilar membrane at a different location in the cochlea. This fuid coupling contributes to the traveling wave propagation on the basilan membrane. Consequently, it contributes importantly to the DPOAE patterns as observed by Knight and Kemp (2000) and Schneider of al (2003), since these patterns reflect both forwand and reverse travel of acoustic excitation along the basilar membrane.

In contrast with the cochlea, the AP is not between two fluid-filled camals. Therefore, coupling between the different sections of this papilla must be different, where some sort of coupling via the tectorial membrane is most likely. As sound enters the AP from the saccular space, it moves along the principal axis of the papilla. Two modes of excitation of the tectorial membrane can be consiclered (Lewis and Leverenz, 1983): [1] fluid How in the recess of the AP may move the tectorial curtain that spans the recess. This excitation may travel down the rest of the tectorium and may thus stimulate the entire papilla. Alternatively, [2] frictional coupling between fluid flow and the tectorial membrane may excite the papilla along its entire length. Since both the tectorial membrane mechanics and the coupling between subsequent 
sections in the papilla is different from that in the cochlea, it is not suprising that the observed DPOAE patterns (this work) differ from those in the cochlea (Knight and Kemp, 2000; Schneider et al., 2003). However, at present, it is impossible to decide what mechanism describes the observed behavior in the AP, as no data are available on the mechanics of the tectorial membrane.

It is important to note that the differences between the amphibian and the mammalian DPOAEs need not reflect a difference in the emission generation mechanism itself. In frogs, the dependence of DPOAE amplitude on stimulus levels is similar to that in mammals (Meenderink and Van Dijk, 2004). This strongly suggests important similarities between DPOAE generation in frogs and mammals. Note that the broad similarities across vertebrate species also include similar neural delays (reviewed in Manley et al., 1990) and similar frequency selectivity in neural tuning (reviewed in Manley, 1990). In conclusion, we showed that the dependence of DPOAE amplitude and phase on the stimulus frequencies is conspicuously different from that in mammals. Our DPOAE measurements did not give any evidence for a mammalian-like traveling wave in the frog inner ear. For the frog's basilar papilla, this is not surprising and our results are consistent with the view that this papilla essentially functions as a single auditory filter. For the amphibian papilla a traveling wave on the tectorial membrane has been hypothesized in the past. This hypothesis is not supported by our results. The differences described here need not reflect fundamental differences between DPOAE generation mechanisms, but presumably reflect the different mechanical properties of the auxiliary structures between the amphibian and basilar papilla and the mammalian cochlea.

\subsection{Acknowledgements}

This work was supported by grants from the Netherlands Organization for Scientific Research (NWO), and the Heinsius Houbolt Foundation to SWFM and PvD, and NIH grant DC-00222 to PMN. 
Chapter 2

\section{Level dependence of distortion}

\section{product otoacoustic emissions in \\ the leopard frog, Rana pipiens pipiens}

\section{Abstract}

The inner ear of frogs holds two papillae specialized in detecting airborne sound, the amphibian papilla (AP) and the basilar papilla (BP). We measured input-output (I/O) curves of distortion product otoacoustic emissions (DPOAEs) from both papillae, and compared their properties. As in other vertebrates, DPOAE I/O curves showed two distinct segments, separated by a notch or kneepoint. The slope of the low-level segment was conspicuously different between the AP and the BP. For DPOAE I/O curves from the AP, slopes were $\leq 1 \mathrm{~dB} / \mathrm{dB}$, similar to what is found in mammals, birds and some lizards. For DPOAE I/O curves from the BP these slopes were much steeper $(\approx 2 \mathrm{~dB} / \mathrm{cB})$. Slopes found at high stimulus levels were similar in the $\mathrm{AP}$ and the $\mathrm{BP}(\approx 2 \mathrm{~dB} / \mathrm{dB})$. This quantitative difference between the low-level slopes for DPOAEs from the AP and the BP may signify the involvement of different mechanisms in low-level DPOAE generation for the two papillae, respectively. 


\subsection{Introduction}

When the ear is stimulated with two primary tones with appropriately chosen frequencies $\left(f_{1}, f_{2}\right)$ and levels $\left(L_{1}, L_{2}\right)$, distortion product otoacoustic emissions (DPOAEs) can be recorded outside the ear. DPOAEs have been reported in mammals (e.g. Kemp, 1979), birds (Taschenberger and Manley, 1998), reptiles (Manley et al., 1993), amphibia (Van Dijk and Manley, 2001), and insects (Coro and Kössl, 2001). They are a manifestation of the nonlinear mechanisms involved in the processing of acoustic stimuli in the inner ear.

Across species, DPOAEs exhibit sexeral similarities. In small mammals, reptiles and insects, slopes of input-output ( $1 / 0)$ curves for DPOAEs at $2 f_{1}-f_{2}$ depend on the levels of the stimulus tones (mammals: Browm, 1987; reptiles: Manley et al., 1993; insects: Coro and Köss1, 2001). For low-and high-level stimulus levels, DPOAE amplitude grows with a relatively constant slope with increasing stimulus levels. For intermediate stimulus levels, these slopes are decreased, resulting in either a kneepoint (slope remains positive) or notch (slope becomes negative) in the $1 / O$ curves. The DPOAE $1 / O$ curves can be modelled as being the result of two DPOAE components (Whitehead et al, 1992). One component is dominant at high stimulus levels. We will refer to this component as the high-level DPOAE. The other component is dominant for low stimulus levels, but saturates for higher stimulus levels. "This component will be referred to as the low-level DPOAE.

Several experiments, performed in mammals (Kim, 1980; Mills, 1997), reptiles (Manley et al., 1993), birds (Froymovich et al., 1995), amphibia (Van Dijk et al., 2003) and insects (Coro and Kössl, 2001) have shown that low-level DPOAEs are more vulnerable to inner ear insult or death than high-level DPOAEs. This suggests that DPOAEs are not only the composite of two components, but that these components are generated by two different mechanisms as well. In mammals, the low-level DPOAE is associated with the nonlinear outer hair cell mechanics, while the bigh-level DPOAE could be due to the passive mechanical properties of the basilar membrane (Mills et al., 1993). This idea of two emission components has been snccessfully incorporated in a model by Mills (1997). In this model, the low-level DPOAE arises around the peaks of the traveling waves of the two stimulus tones (his "peak" component), while the high-level DPOAE originates more basally in the cochlea (his "basal" component). Other models (e.g. "Talmadge et al, 1998) that also incorporate two distinct emission components are only valid for low stimulus levels, and therefore not applicable for sinulating DPOAE I/O curves. These modals are able to predict other phenomena observed in DPOAE data for low stimulus levels (e.g. DPOAE fine-structure).

More recently, it has been shown that the presence of notches in $1 / 0$ curves, 
and the differential vilnerability of high-and low-level DPOAES may arise from a single nonlinearity (Lukashkin and Russell; 1998, 1999). The model calculations in these papers show that a Boltzman function, which is used to describe hait cell transduction, is sufficient to produce notches in amplitude data. In order to simulate the differential vulnerability of high-and low-level DPOAEs, the incomporation of a positive feedback mechanism in this model is necessary. Thus, both the two source (i.e. Mills, 1997) and the one-source (i.e. Lukashkin and Russell, 1998, 1999) descriptions were successfully applied to describe DPOAEs from the mammalian cochlea.

The anatomy of the frog's inmer ear differs considerably from the mammalian ear, the most prominent difference being the absence of a cochlea in amphibians. Instead, the inner ear of a frog holds two distinct papillate that are sensitive to airborne sound. The amphibian papilla (AP) consists of an S-shaped stip of hair cells, covered by a tectorial membrane (Frishkopf and Flock, 1974). It exhibits a tonotopic organization (Lewis et $a l ., 1982$ ), and the presence of traveling waves in the tectorial membrane has been proposed (Hillery and Narins, 1984). In Rana pipiens pipiens, afferent. neurons from the AP are tuned to frequencies in the range of $0.1-1.25 \mathrm{kHz}$ (Ronken, 1991). The basilar papilla (BP) is a much simpler structure. It consists of a small patch of sensory epithelium, partly covered by a tectorial membrane. The BP is of special interest to hearing research because it functions as a single auditory filter: almost all nerve fiber units ane tuned to the same frequency (Ronken, 1991), there is no evidence for traveling waves, and in ranid frogs the polarity of stereovillar bundles is identical for all hair cells (Lewis, 1978). In neither of these papillae is the sensory epithelium over a basilar membrane. Rather, hair cells are directly on a rigid support structure.

In the present study amplitude and phase of DPOAEs recorded from both the $\mathrm{AP}$ and the BP are presented. We will discuss to what extent our results aro similar to those in other species, and whether mammalian models (Mills, 1997, Lukaslikin. and Russell, 1998,1999 ) are applicable to the amphibian ear.

\subsection{Materials and Methods}

The results presented were obtained. from recordings in the right ears of five leopard. frogs, Rana pipiens pipiens. Across animals, body weight ranged from 35.5 to $58.6 \mathrm{~g}$ (average $46.4 \mathrm{~g}$ ), and snout-vent length ranged from 7.4 to $8.4 \mathrm{~cm}$ (average $8.1 \mathrm{~cm}$ ). In order to immobilize the animals, they were andesthetized via an intramuscular injection of pentobarbital (Nembutal: $30 \mathrm{mg} / \mathrm{kg}$ ) in one of the hindlimbs. During the experiment, the frog was covered by gauze soaked in tap water bo prevent dehydration. Experiments were performed in a sound-attentiation room. All procedures. 
were approved by the animal experiment committee of the Maastricht University.

A custom-built emission recording probe was placed against the skin surrounding the frog's tympanic membrane. To reduce acoustic energy loss, silicone grease was used to obtain a tight seaj between the probe and the skin. The probe contained two miniature speakers (E-A-RTONE 3A; Aero Company; IN; USA) for stimulus delivery, and a $1^{\prime \prime}$ high-sensitivity condenser microphone (B\&K type 4179 ; Brüel \& Kjar; Demmark) for emission recording. Stimulus tones, with frequencies $f_{1}$ and $f_{2}\left(f_{2}>f_{1}\right)$ were generated from two separate D/A channels (RP2; Tucker-Davis Technologies; Gainesville; FL; USA). The levels of the two stimulus tones ( $L_{1}$ and $L_{2}$, respectively) were controlled by separate programmable attenuators (PA5; TDT; Gainesville; FL; USA) and played through the miniature speakers.

The microphone signal was passed through a low-noise amplifier (B\&K type 2660; Brüel \& Kjær; Denmark; amplification $=20 \mathrm{~dB}$ ) and a measuring amplifier (B\&K type 2610; Brüel \& Kjær; Denmark; amplification $\geq 10 \mathrm{~dB}$ ). The amplified signal was recorded via an A/D channel (RP2; TDT; Gainesville; FL; USA). The digitized microphone signal was stored on computer disc to be available for offline analysis. In order to monitor DPOAEs during the experiment the frequency spectrum of the amplified microphone signal was viewed online with a spectrum analyzer (SR760; Stanford Research Systems; Sunnyvale; CA, USA). Customized software, developed in MATLAB (The MathWorks, Inc; Natick; MA, USA.) and RPvds (TDT, Gainesville; FL; USA) was used to control stimulus tone generation and signal recording.

To determine the nonlinear distortion of the microphone and other recording equipment, measurements were conducted with the open end of the probe pressed against a hard surface. Across frequencies system distortion was about $90 \mathrm{~dB}$ below the primaries. Only for the lowest stimulus levels used $\left(L_{1}=L_{2}<55 \mathrm{~dB}\right.$ SPL) did the system distortion disappear into the estimated noise floor. All DPOAEs were corrected for system distortion by vector-subtracting system distortion [as determined against the hard surface] from the stored microphone signals.

Primary frequency $f_{1}$ varied between 4.13 and $3212 \mathrm{~Hz}$ in approximately $100 \mathrm{~Hz}$ steps, while the frequency ratio $f_{2} / f_{1}$ was kept constant at 1.1. For each $f_{1}$, the stimulus level was decreased in $1 \mathrm{~dB}$ steps from $90 \mathrm{~dB}$ SPL down to stimulus levels for which DPOAEs were indistinguishable from the noise floor $\left(L_{1}=L_{2}=51-41 \mathrm{~dB}\right.$ SPL). Only equal primary levels were used. The recording system was calibrated in situ using white noise at the starti of the experiment.

For each parameter combination $\left(f_{1}, L_{1}, f_{2}, L_{2}\right)$ the stimulus tones were played continuously for 50,000 sample points $(\approx 4.10 \mathrm{sec}$, with a sample frequency of 12.2 $\mathrm{kH}_{2}$ ). The frequency $f_{1}$ was chosen so that an integer number of sample points contained exactly 100 periods of this stimulus tone. Because the frequency ratio 
$f_{2} / f_{1}$ was constant at 1.1 , not only $f_{1}$, but also all other frequencies of interest were exactly periodic over the same number of sample points (i.e. 110 periods for $f_{2}, 90$ periods for $2 f_{1}-f_{2}$, etc.). Depending on stimulus frequency $f_{1}$, the 50,000 sample points contained a variable number of blocks in which $f_{1}$ had 100 periods (e.g. 16 blocks for $f_{1}=413 \mathrm{~Hz}$ and 131 blocks for $f_{1}=3213 \mathrm{~Hz}$.)

The recorded microphone signal can be segmented in a fashion similar to the stimulus. From the recorded 50,000 sample points, the first periodic block and the trailing sample points, which did not make up an entire periodic block, were omitted from the analysis. The amplitudes $\left(L_{1}, L_{2}\right)$ and phases $\left(\phi_{1}, \phi_{2}\right)$ of the two stimulus tones were calculated using Fourier analysis. The parameters thus obtained were used to subtract the stimulus tones from the microphone signal in the time domain, leaving a signal containing only the DPOAEs and system noise. The periodic blocks in the remaining signal were subjected to a level-crossing artifact rejection method. Artifact free blocks were sub-averaged in two buffers $A$ and $B$. The average $(A+B) / 2$ can be used to estimate the DPOAEs amplitudes and phases, while the difference A-B provides an estimate for the noise levels. The amplitude and phase of DPOAEs were calculated using Fourier analysis.

In these experiments, the phase of the stimulus tones was not controlled. To correct for phase variations of the stimulus tones across recordings, phase $\Phi_{n f_{1}-m f_{2}}$ of the DPOAEs will be given relative to the calculated phases of the stimulus tones:

$$
\Phi_{n f_{1}-m f_{2}}=\phi_{n f_{1}-m f_{2}}-\left(n * \phi_{1}-m * \phi_{2}\right),
$$

where $\phi_{n f_{1}-m f_{3}}$ is the recorded DPOAE phase, and $\phi_{1}$, and $\phi_{2}$ are the estimated phases of the primaries.

\section{$2.3 \quad$ Results}

\subsubsection{DPOAE-audiograms}

Distortion product otoacoustic emissions were detectable in all frogs investigated. An example of the DPOAE spectrum $\left(f_{1}=1822 \mathrm{~Hz} ; L_{1}=L_{2}=85 \mathrm{~dB}\right.$ SPL $)$ is displayed in Fig. 2.1. Besides the large pealks corresponding to the stimulus tones, several distortion products are visible. In the present study the recorded data was systematically analyzed for distortion products at frequencies $2 f_{1}-f_{2}, 3 f_{1}-2 f_{2}, 2 f_{2}-$ $f_{1}$ and $3 f_{2}-2 f_{1}$ (emboldened in Fig. 2.1). Fig. 2.2 shows DPOAE-audiograms of $2 f_{1}-f_{2}, 3 f_{1}-2 f_{2}, 2 f_{2}-f_{1}$ and $3 f_{2}-2 f_{1}$ DPOAEs for one animal. In general, amplitudes for DPOAE at $2 f_{1}-f_{2}$ and $2 f_{2}-f_{1}$ were larger than for $3 f_{1}-2 f_{2}$ and $3 f_{2}-2 f_{1}$. 


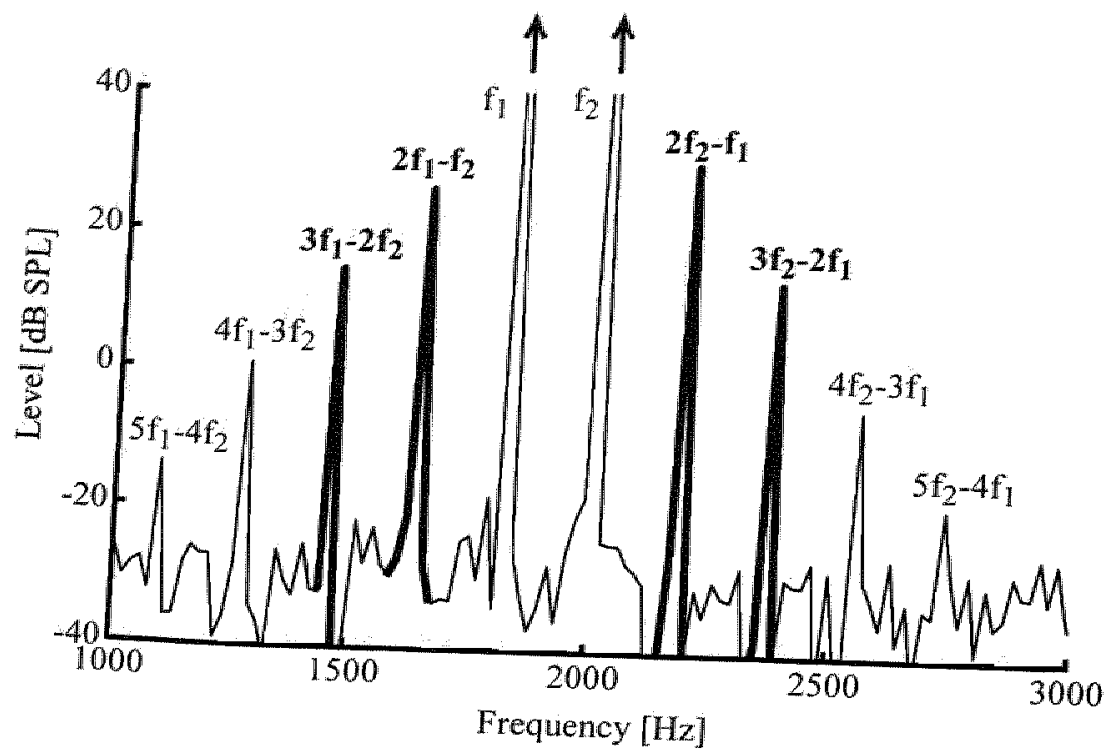

Figure 2.1: Spectrum of the averaged microphone signal, showing several DPOAE peaks from the basilar papilla of $R$. pipiens pipiens; $f_{1}=1822 \mathrm{~Hz}, f_{2} / f_{1}=1.1, L_{1}=L_{2}=85$ dB SPL. The spectral peaks corresponding to the stimulus tones exceed the vertical scale (arrows). The combination tones are labelled by their stimulus tones DPOAEs were observod at recorded data were and $3 f_{2}-2 f_{1}$. These DPOAEs are andized for DPOAEs at $2 f_{1}-f_{2} 3 f_{1}-2 f_{2}, 2 f_{2}-f_{1}$ spectiral peaks.

DPOAE-audiograms showed a bimodal dependence of DPOAE level on frequency. Emission amplitudes were relatively small near $f_{d p}=1250 \mathrm{~Hz}$. The DPOAE-audiogram was similar in all animals studied. Based on characteristic frequencies of auditory nerve fibers from the AP and the BP (Ronken, 1991), we will refer to emissions with a frequency below $1250 \mathrm{~Hz}$ as AP-DPOAE (amphibian papilla DPOAE), and with frequencies above $1250 \mathrm{~Hz}$ as BP-DPOAE (basilar

\subsubsection{Amplitude and phase of $2 f_{1}-f_{2}$}

In Fig. $2.3 \mathrm{a}, 1 / \mathrm{O}$ curves for $f_{d p}=2 f_{1}-f_{2}$ are plotted in a three-dimensional format. In this figure, and all subsequent figures, only data points exceeding the estimated amplitude of the DPOAEs increases with increasing stimulus levels. An exception
to this is the I/O curve at $f_{d y}=908 \mathrm{~Hz}$, which shows a notch in amplitude around 


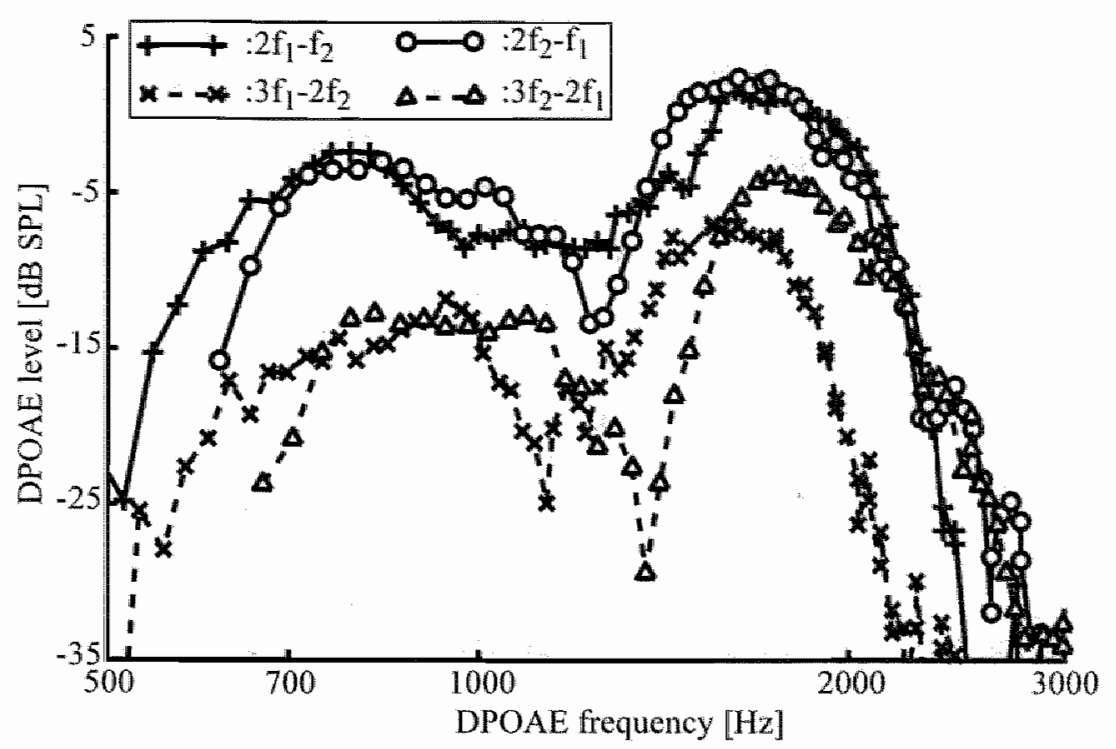

Figure 2.2: Levels of distortion products at frequencies $2 f_{1}-f_{2}\left({ }^{\prime}\right.$ ', solid line), $3 f_{1}-2 f_{2}$ ("X', dashed line), $2 f_{2}-f_{1}\left({ }^{\prime} O^{\prime}\right.$, solid line), $3 f_{2}-2 f_{1}(' \Delta$ ', dashed line) as a function of distortion product frequency. The DPOAE-audiograms were recorded with $L_{1}=L_{2}=70$ $\mathrm{dB}$ SPL, and $f_{2} / f_{1}=1.1$. Noise floor is approximately $-33 \mathrm{~dB}$ SPL.

stimulus levels of $69 \mathrm{~dB}$ SPL.

The data are redrawn in Fig. $2.3 \mathrm{~b}$ in the form of a contour plot. This figure shows that the bimodal dependence of DPOAE level on frequency, as seen in Fig. 2.2 for $L_{1}=L_{2}=70 \mathrm{dBSPL}$, is not restricted to that particular stimulus level but present for all stimulus levels used. For high and intermediate stimulus levels $\left(L_{1}=L_{2} \geq 65\right.$ dB SPL) the contour lines exhibit an inflection around $f_{d p}=1250 \mathrm{~Hz}$. For low stimulus levels $\left(L_{1}=L_{2} \leq 65 \mathrm{~dB}\right.$ SPL) there is a region around $1250 \mathrm{~Hz}$ where DPOAE level does not exceed the estimated noise floor, although for both lower and higher frequencies, DPOAE are still detectable.

Visible in Fig. 2.3, but more clearly illustrated in Fig. 2.4a, is a conspicuons difference between AP-DPOAE and BP-DPOAE 1/O curves.

Based on their slopes, $1 / 0$ curves can be divided into two segments. For the AP-DPOAE $1 / 0$ curves, the segments are separated by a kneepoint at 70 aB SPL stimulus levels. At levels above the kneepoint, the DPOAE amplitude grows steeply (2 $\mathrm{dB} / \mathrm{dB}$ ) with stimulus amplitude. Below the kneepoint, the DPOAE amplitude decreases with a shallow slope $(<1 \mathrm{~dB} / \mathrm{dB})$, into the noise floor. At some $\mathrm{AP}^{*}$ frequencies the two segments were separated by a notch, rather than a kmeepoint (for example Fig. 2.3a, I/O curve at $f_{d p}=908 \mathrm{~Hz}$ ). In those cases the amplitude 


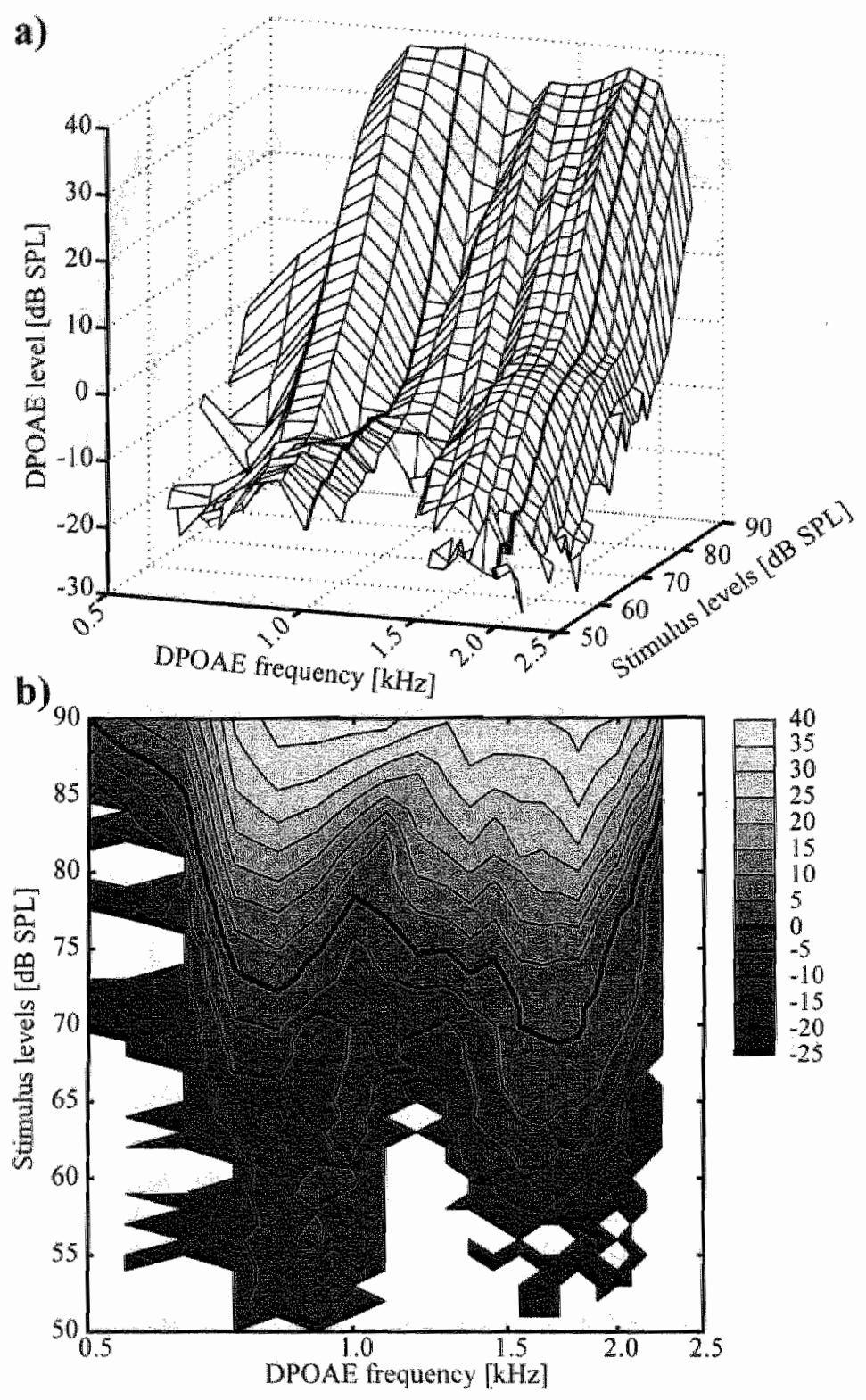

Figure 2.3: (a) 1/O curves of DPOAEs at $2 f_{1}-f_{2}$ in a three-dimensional format. Stimulus frequency ratio was fixed $f_{2} / f_{1}=1.1$, while stimulus levels $L_{1}=L_{2}$ were varied in $1 \mathrm{~dB}$ steps. Only data points exceeding the estimated noise floor by at least $12 \mathrm{~dB}$ are shown. The emboldened $1 / 0$ curves are shown again in Fig. 2.4a. (b) Data from (a) redrawn in the form of a two-dimensional contour plot. Contour lines are plotted at $5 \mathrm{~dB}$ intervals. As a reference the $0 \mathrm{~dB}$ SPL contour line is emboldened. 


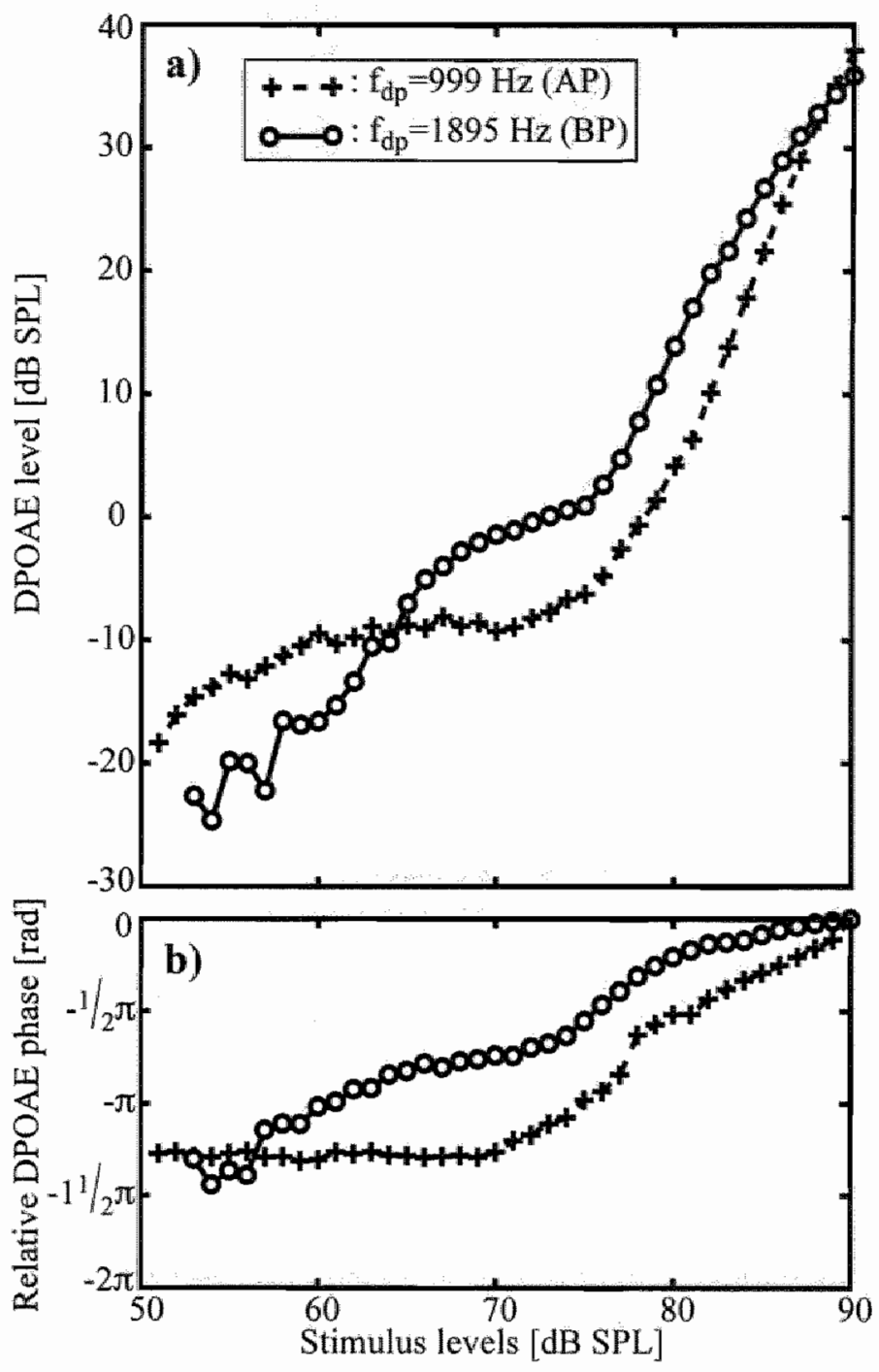

Figure 2.4: (a) Individual DPOAE I/O curves for one AP-DPOAE frequency $\left(f_{i j}=999\right.$ $\mathrm{H} z$; ' + ', dashed line) and one BP-DPOAE frequency $\left(f_{d p}=1895 \mathrm{H} /\right.$; ' $O$ ', solid line). (b) Corresponding relative phase of the DPOAE. The phase has been set to zero at $L_{1}=L_{2}=$ $90 \mathrm{~dB}$ SPL. Curves correspond to the emboldened lines in Figs. 2.3a and 2.5.

of the DPOAE below, the notch initially increases with decreasing stimulus levels. The shallow and steep slopes for low and high stimulus levels, respectively, are characteristic of the AP.

In contrast, the BP-DPOAE amplitude grows steeply with stimulus level $(>1$ $\mathrm{dB} / \mathrm{dB}$ ) for both low- and high-level stimuli. The low-and high-level segments were 


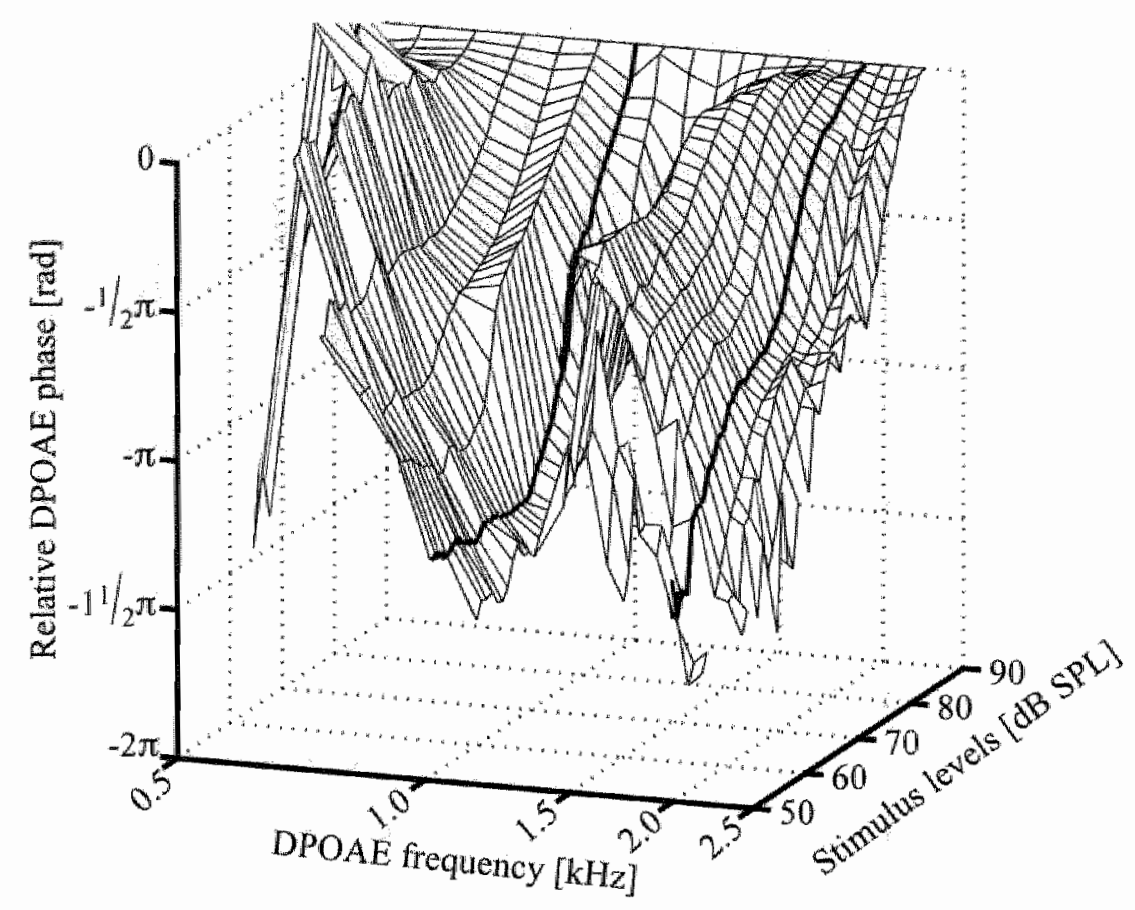

Figure 2.5: (a) Relative phase of the DPOAE 1/O curves from Fig. 2.3 in a threedimensional format. For each $1 / O$ curve the phase has been unwrapped by removing $2 \pi$ phase jumps. The urwrapped phase has been set to zero at $L_{1}=L_{2}=90 \mathrm{~dB}$ SPL. The emboldened phase curves are shown again in Fig. $2.4 \mathrm{~b}$.

separated by a knecpoint (at 77 dB SPL stimulus level in Fig. 2.4a).

Fig. 2.4b shows the relative phase of the DPOAE I/O curves in Fig. 2.4a. Phase chata of individual $1 / 0$ curves was unwrapped by removing $2 \pi$ phase jumps and the phase was arbitrarly set to zero at $L_{1}=L_{2}=90 \mathrm{~dB} \mathrm{SPL}$. A close relation between the amplitude and phase can be seen. For stimulus levels associated with the notch/kneepoint in an 1/O curve the phase changed rapidly. In contrast, in the stimulus level ranges for which the DPOAE amplitude grew with a constant slope the phase varied relatively litte.

A complete overview of the relative phase corresponding to the DPOAE $1 / 0$ curves from Fig. 2.3 is given in Fig. 2.5. For low stimulus levels, the phase increasingly lagged the primary tones with increasing frequency, both for AP-DPOAE and $B P$-DPOAE. In other words, with decreasing stimulus levels the DPOAE group delay, defined as $\tau=-\frac{1}{2 \pi} d \phi / d f$, of phase versus frequency curves, increased.

The phase characteristics for individual DPOAE I/O curves are similar over the entire frequency range. For each DPOAE I/O curve two segments can be seen 
where the phase changed relatively little. The transition between these segments was characterized by a rapidly changing phase.

\subsubsection{Amplitude and phase of other DPOAEs}

Figs. 2.6 and 2.7 give an overview of the amplitude and associated phase data at DPOAE frequencies other than $2 f_{1}-f_{2}$. Besides the ranges of the frequency axes, the format of these figures is the same as those in Figs. 2.3 and 2.5. As was also shown in Fig. 2.2, amplitudes at $2 f_{1}-f_{2}$ and $2 f_{2}-f_{1}$ were larger than at $3 f_{1}-2 f_{2}$ and $3 f_{2}-2 f_{1}$. However, several similarities existed across the distortion components. [1] All DPOAE I/O curves showed an increase in amplitude with increasing stimulus levels. [2] This growth was with a constant slope for low and high stimulus levels. For intermediate stimulus levels the slopes of DPOAE $1 / 0$ curves decreased, resulting in either a notch or a kneepoint. [3] For intermediate stimulus levels, associated with the decreased slope of the $1 / O$ curve, the phase changed rapidly. Away from intermediate stimulus levelis, this change in phase was mone gradual or absent. [4] The group delay of DPOAE-audiograms increased with decreasing stimulus levels.

There were clear differences between emissions with $f_{d_{p}}<f_{1}, f_{2}$, i.e. $2 f_{1}-f_{2}$ and $3 f_{1}-2 f_{2}$, and emissions with $f_{d p}>f_{1}, f_{2}$, z.e. $2 f_{2}-f_{1}$ and $3 f_{2}-2 f_{1}$. In the BP frequency region the tuming of individual contour limes (Figs. 2.3b and 2.6) was much wider for DPOAE at $2 f_{1}-f_{2}$ and $3 f_{1}-2 f_{2}$ compared to DPOAE at $2 f_{2}-f_{1}$ and $3 f_{2}-2 f_{1}$. For instance, the widths of the $0 \mathrm{~dB}$ SPL contour lines (emboldened black lines in Figs. 2.3b and 2.6) at $5 \mathrm{~dB}$ above the minimum were $452 \mathrm{~Hz}$ and $440 \mathrm{~Hz}$ for $2 f_{1}-f_{2}$ (Fig. 2.3b) and $3 f_{1}-2 f_{2}$ (Fig. 2.6b), respectively. For $2 f_{2}-f_{1}$ (Fig. 2.6a) and $3 f_{2}-2 f_{1}$ (Fig, 2.6c) these widths were $731 \mathrm{~Hz}$ and $1208 \mathrm{~Hz}$, respectively. Besides this difference in tuning widths, a clear separation between AP-DPOAE and BP. DPOAE can be seen for $2 f_{1}-f_{2}\left(\right.$ Fig. 2.3b) and $3 f_{1}-2 f_{2}$ (Fig. 2.6b). For DPOAE at $2 f_{2}-f_{1}$ (Fig. 2.6a) and $3 f_{2}-2 f_{1}$ (Fig. 2.6c) this separation is absent; DPOAE amplitude does not decrease when crossing from AP-DPOAE to BP-DPOAE.

A comparison between the various DPOAEs $\left(2 f_{1}-f_{2}, 2 f_{2}-f_{1}, 3 f_{1}-2 f_{2}\right.$, and $3 f_{2}-2 f_{1}$ ) ewoked with a particular set of stimulus frequencies, showed that for each DPOAE frequency the transition from the low-level to the high-level segment occurred at a different stimulus level. This is illustrated in Fig. 2.8 for one set of DPOAE $1 / 0$ curves from the basilar papilla $\left(f_{1}=1822 \mathrm{H} z\right)$. Here, each $\mathrm{I} / \mathrm{O}$ curve exhibits a kneepoint or a notch at different stimulus levels: $2 f_{1}-f_{2}: 69 \mathrm{~dB} \mathrm{SPL}$; $2 f_{2}-f_{1}: 72 \mathrm{~dB} \mathrm{SPL} ; 3 f_{1}-2 f_{2}: 74 \mathrm{~dB}$ SPL; $3 f_{2}-2 f_{1}: 76 \mathrm{~dB}$ SPL. This can also be inferred from the various contour plots (Figs. $2.3 \mathrm{~b}$ and 2.6 ). The transition from low-level to high-level DPOAE is marked by a decrease in the slope of the $1 / O$ curve, which is reflected in a larger distance between the contour lines in the contour plots. 


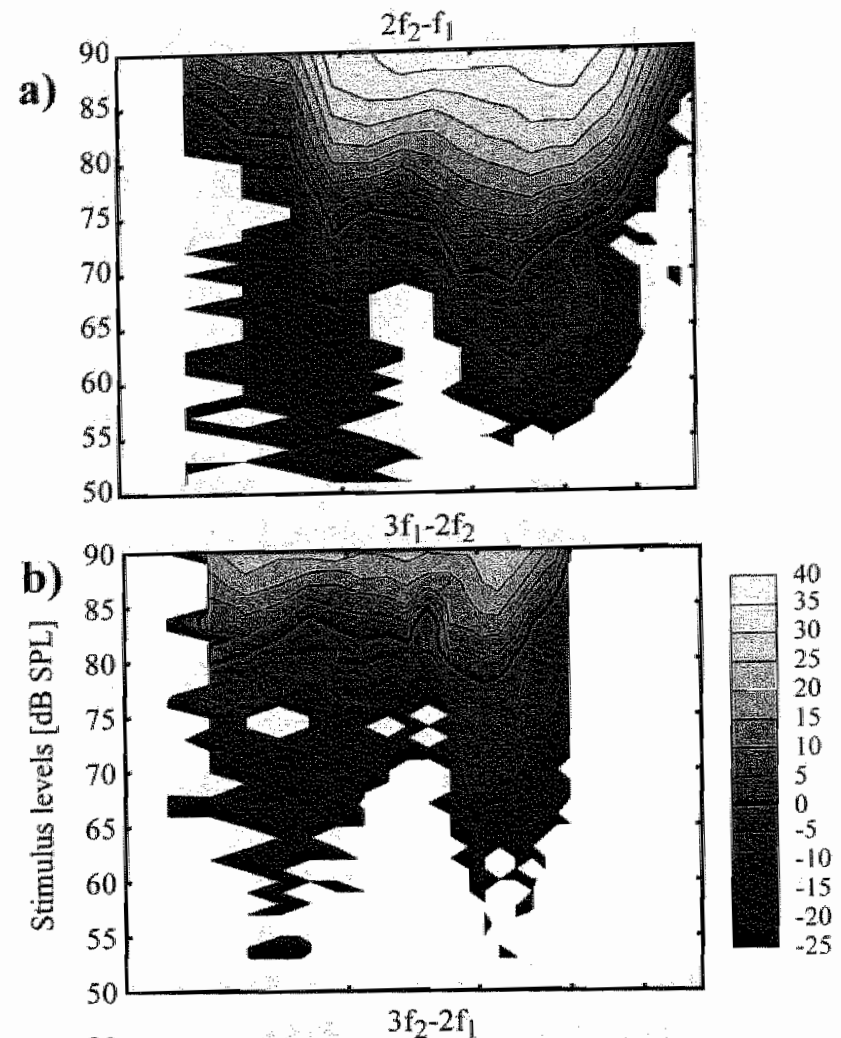

c)

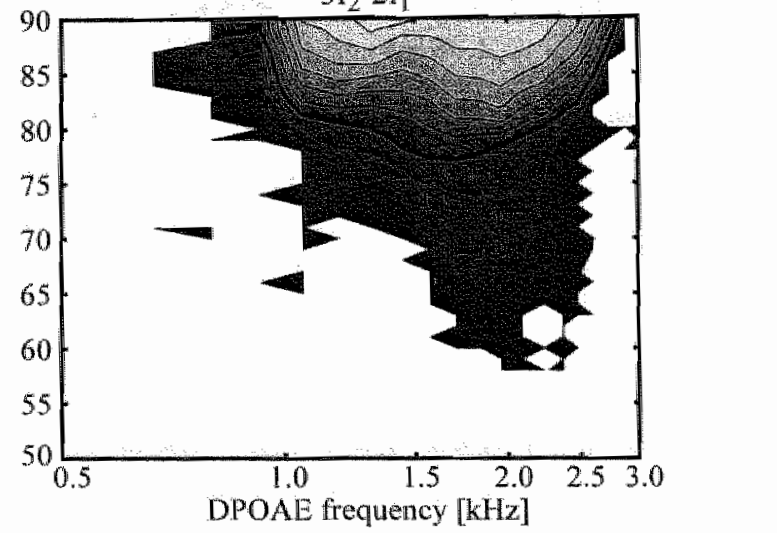

Figure 2.6: Anplitude of several DPOAEs in the form of two-dimensional contour plots. Except for the frequency range, the format is the same as in Fig $2.3 \mathrm{~b}$. (a) $2 f_{2}-f_{1}$, (b) $3 f_{1}-2 f_{2},(c) 3 f_{2}-2 f_{1}$. Amplitude contour lines are drawn at 5 dB intervals. For reference the $0 \mathrm{~dB}$ SPL contour line is emboldened. 

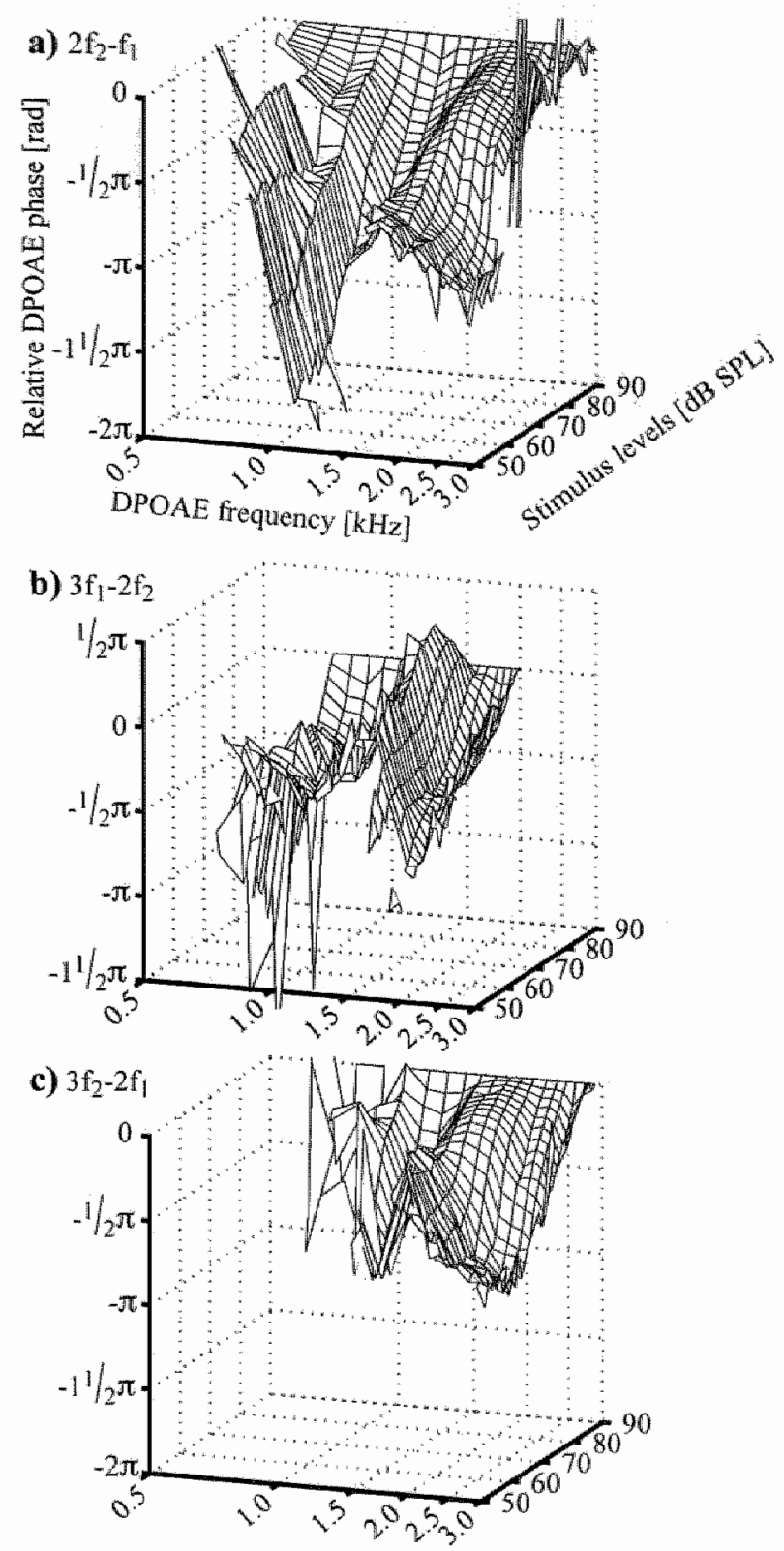

Figure 2.7: Relative phase of the DPOAE 1/O curves from Fig. 2.6 in a three-dimensional format. 'The displayed frequency range is different from that in Fig. 2.4. (a) $2 f_{2}-f_{1}$, (b) $3 f_{1}-2 f_{2}$, (c) $3 f_{2}-2 f_{1}$. As in Fig. 2.5 , the phase has been unwrapped, and the phase thus obtained has been set to zero at $L_{1}=L_{2}=90 \mathrm{~dB} \mathrm{SPL}$. 


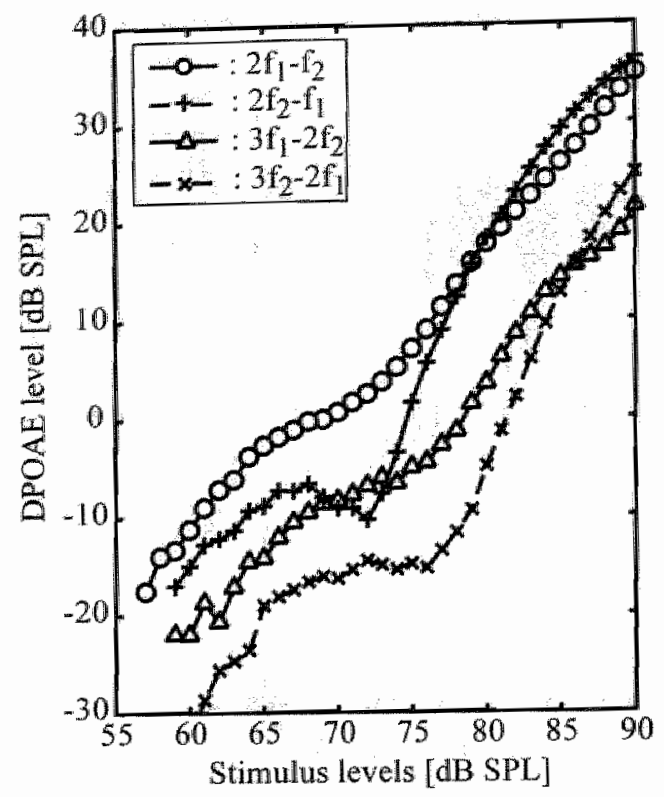

Figure 2.8: 1/O curves for several DPOAEs from the basilar papilla, all evoked with the same set of stimulus tones; $f_{1}=1822 \mathrm{~Hz}, f_{2} / f_{3}=1.1,2 f_{1}-f_{2}=1640 \mathrm{~Hz}\left(\mathrm{O}^{\prime}\right.$; solid line); $2 f_{2}-f_{1}=2186 \mathrm{~Hz}$ (' + ', dashed line); $3 f_{1}-2 f_{2}=1458 \mathrm{~Hz}$ ( $\triangle$ ', solid line); $3 f_{2}-2 f_{1}=2369 \mathrm{~Hz}$ ( $x$ ", dashed line). Based on their slopes, $1 / 0$ curves can be diwided into two segments; one segment is evoked with low level stimulus tones and the ollher with high level stimulus tones. For the different DPOAEs, the transition from one segment to the other occurs at different levels of the stimulus tones: $2 f_{1}-f_{2}: 69 \mathrm{~dB} \mathrm{SPL} ; 2 f_{2}-f_{1}: 72$ $\mathrm{dB}$ SPL; $3 f_{1}-2 f_{2}: 74 \mathrm{~dB}$ SPL; $3 f_{2}-2 f_{1}: 76 \mathrm{~dB} \mathrm{SPL}$.

In Figs. $2.3 \mathrm{~b}$ and 2.6 the contour lines are further apart ctound those stimulus levels for which the DPOAE amplitude is approximately 0 dB SPL (emboldened black lines). This emboldened black line occurs at different stimulus levels in the different contour plots, indicating that the transition from low-level to high-level DPOAE occurs at diferent stimulus levels for the different DPOAEs.

\subsubsection{Slopes of DPOAE $1 / O$ curves}

Commonly, slopes of DPOAE I/O wrves are determined by fitting straight lines to those parts of the $\mathrm{I} / \mathrm{O}$ curve for which the the slope is approximately constant (e.g. Mills and Rubel, 1996; Coro and Köss, 2001). This method requires the choice of a stimulus level range for which the DPOAE $1 / O$ curve is assumed to be linear. To avoid this subjective definition of stimulus ranges we calculated the slope of the 


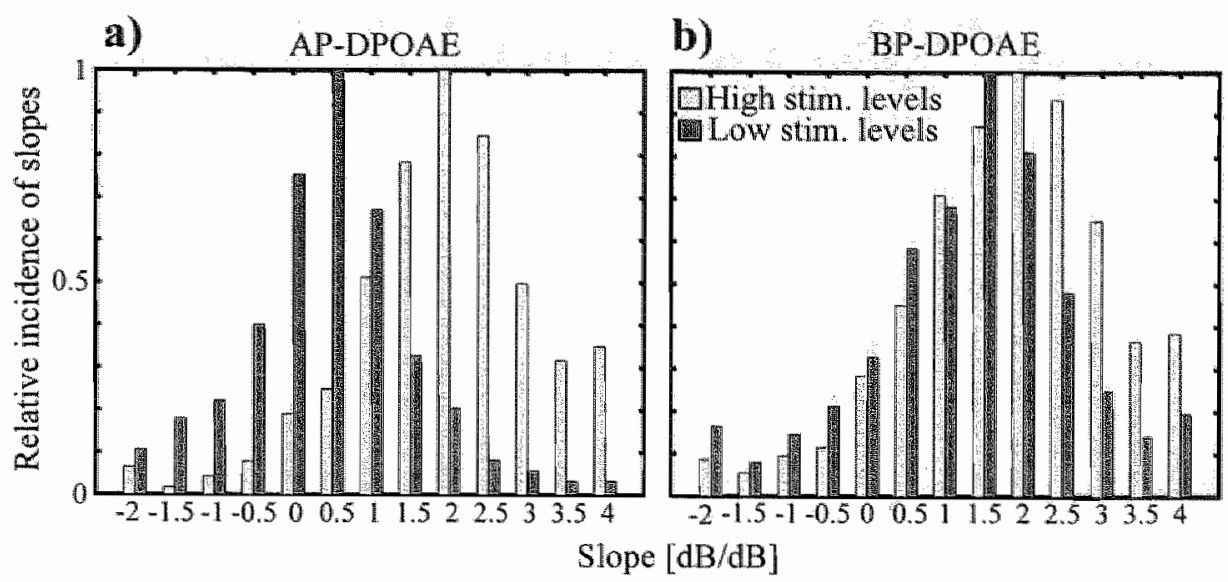

Figure 2.9: Comparison of the growth rate of DPOAEs from the AP and the BP. DPOAE I/O curves were grouped as being either AP-DPOAE $\left(f_{d y}<1250 \mathrm{~Hz}\right)$ or BP-DPOAE $(f d p>1250 \mathrm{~Hz})$. Within each group individual DPOAE $1 / \mathrm{O}$ curves were divided in two subgroups: stimulus levels $>70 \mathrm{~dB}$ SPL and stimulus levels $<65 \mathrm{~dB}$ SPL. (a) Distribution of observed slopes of high and low stimulus levels for the amphibian papilla. Average slopes: high stimulus levels: $1.96 \mathrm{~dB} / \mathrm{dB}$; low stimulus levels: $0.44 \mathrm{~dB} / \mathrm{dB}$. (b) same as (a), but for the basilar papilla. Average slopes: high stimulus levels: $1.82 \mathrm{~dB} / \mathrm{dB}$; low stimulus levels: $1.30 \mathrm{~dB} / \mathrm{dB}$. Histograrns were scaled to a peak value 1 .

DPOAE $\mathbb{1} / O$ curve in each data point $X$ by fitting a straight line through its two neighbouring points (i.e. the data points evoked with stimulus levels 1 dB above and below the levels of $X$ itself).

Slopes, combined for all DPOAE frequencies and all frogs investigated are shown in Fig. $2.9 \mathrm{in}$ histograms. Based on stimulus level, both AP.DPOAE and BP. DPOAE were subdivided in a low-and a high-level part. Fig. 2.90 highlights the difference in slope between low-and high-level slopes for AP-DPOAE. High-level slopes averaged $1.96 \mathrm{~dB} / \mathrm{dB}$ while low-level slopes averaged $0.4 \mathrm{~dB} / \mathrm{dB}$. For BP. DPOAE slopes (Fig. 2.9b) these averages were 1.82 and $1.30 \mathrm{~dB} / \mathrm{dB}$ for high and low-level slopes, respectively.

\subsubsection{Phase difference between low- to high-level DPOAE}

In order to quantify the phase change associated with the transition from low to high-level DPOAE, Fig. 2.10 displays the phase difference between DPOAE evoled with stimulus levels of 60 and $90 \mathrm{~dB}$ SPL, respectively.

Result, for all frogs were combined, where different frogs have different symbols. Each distortion product is represented in a different ponel. Both for AP-DPOAE and BP-DPOAE, and for all distortion products, the phase difference increases sys- 


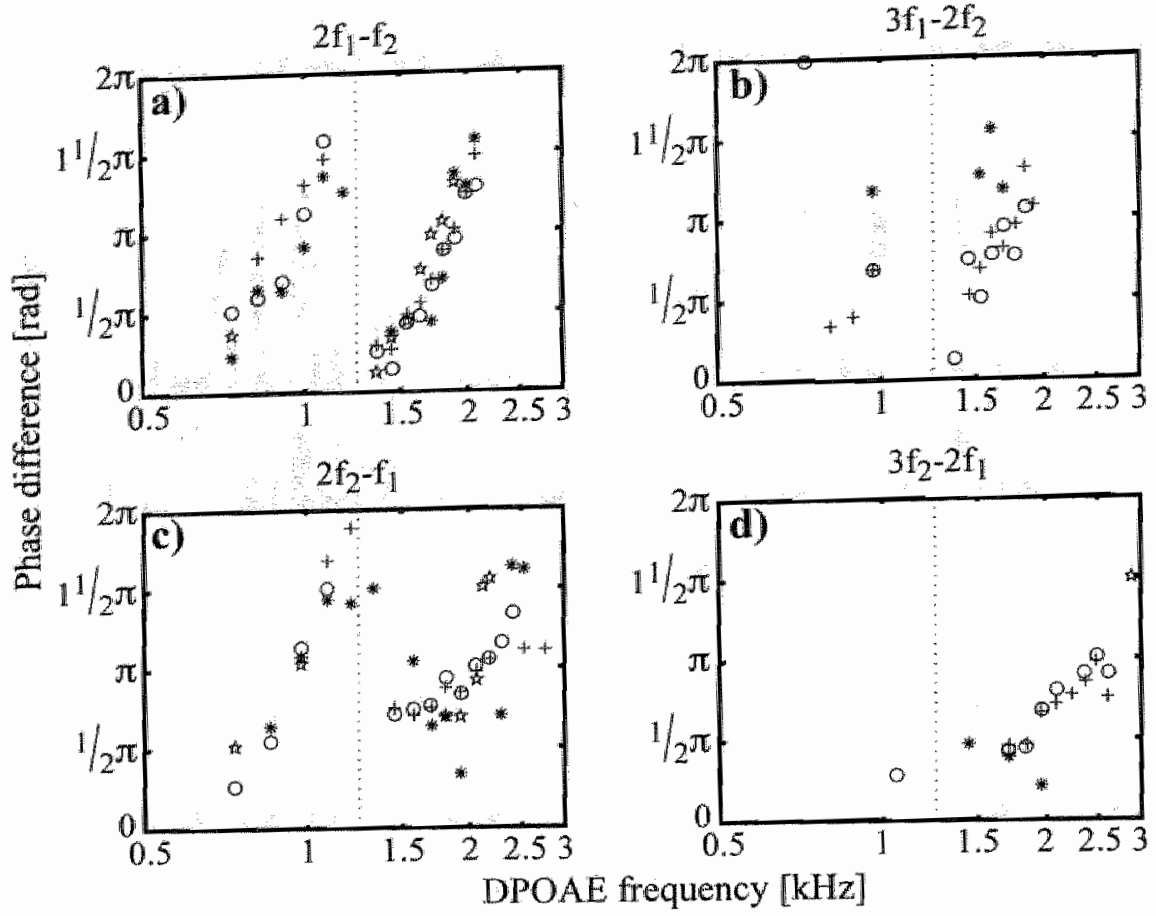

Figure 2.10: Difference between the 60 and $90 \mathrm{~dB}$ SPL DPOAE phase as a function of distortion product frequency. (a) $2 f_{1}-f_{2}$ (b) $3 f_{1}-2 f_{2}$ (c) $2 f_{2}-f_{1}$ (d) $3 f_{2}-2 f_{1}$. Each symbol denotes data from a different frog. The vertical dashed line gives the frequency separation between AP and BP (1250 Hz; Ronken, 1991).

tematically with increasing frequency, For $2 f_{1}-f_{2}, 3 f_{1}-2 f_{2}$ and $3 f_{2}-2 f_{1}$ the phase difference is up to $1.5 \pi$ and for $2 f_{2}-f_{1}$ this difference is up to $2 \pi$.

\subsection{Discussion}

\subsubsection{Bimodal shape of DPOAE-audiograms}

All DPOAEmadiograms (directly reconded or reconstructed from DPOAE I/O curves) obtained in the frog showed a bimodal dependence on frequency. Two frequency regions were found where relative maxima in DPOAE amplitude occurred. The frequency ranges of these two regions coincided with the frequency ranges of the tuning of single nerve fibers from the two hearing papillae. This suggests that, for all stimulus levels investigated, DPOAEs arose in these papilae, and not via other nonlinear processes in the ear. The same conclusion was drawn by Van Dijk and Manley (2001) and Van Dijk et al. (2002). 
Interestingly, DPOAEs were not found for distortion product frequencies below approximately $500 \mathrm{~Hz}$, although the AP is innervated by nerve fibers tuned to frequencies as low as $100 \mathrm{~Hz}$ (Frishkopf and Goldstein, 1963, Frishkopf et al, 1968, Feng et al., 1975). A similar observation was made for low frequency DPOAEs in the treefrog, $H$. cinerea (Van Dijk and Manley, 2001). The absence of low frequency DPOAEs might be related to the fact that the tuning of the low frequency (rostral) part of the AP in R. pipiens pipiens is based on electrical resonance of the hair cells (Smotherman and Narins, 1999a). However, in the bobtail lizard (Manley et al, 1993) DPOAEs have been recorded in the frequency range which probably relies on electrical resonance as tuning mechanism (Manley, 2002). Thus, the absence of DPOAEs may not be a fundamental characteristic of electrical hair cell tuning:

Alternatively, it might be that DPOAEs are generated in the low frequency part. of the AP, but that they are attenuated below detectable levels before being emitted. The tectorial membrane on the rostral portion of the AP is a rather massive structure, which may impede any low frequency DPOAEs generated by the underlying hair cells (Lewis et al., 1982)

\subsubsection{Growth of DPOAE I/O curves}

The data presented in this paper showed that the slopes of DPOAE I/O curves vary with stimulus tone level, similar to what has been found in mammals (e.g. Kim, 1980) and other vertebrates (e.g. Taschenberger and Manley, 1998). We will discuss three possible mechanisms to explain the decreased slopes of DPOAE I/O curves around intermediate stimulus levels in amphibians.

The first way to explain a transition from low- to high-level behavior is a change in vibrational mode of the structures involved in the inner ear. If the mode of vibration changes at some stimulus levels, the transition in DPOAE behavior is expected to occur at this transition stimulus level for all DPOAE orders. In contrast, our data show that the transition from low- to high-level DPOAE takes place at lower stimulus levels for $2 f_{1}-f_{2}$ and $2 f_{2}-f_{1}$ than for $3 f_{1}-2 f_{2}$ and $3 f_{2}-2 f_{1}$ (Fig. 2.8). This observation excludes a vibration-mode transition as the origin of notches/kneepoints in DPOAE I/O curves.

A second explanation for the presence of notches/kneepoints in DPOAE $1 / O$ curves involves the presence of a single DPOAE source. Lukashkin and Rusell (1998, 1999) presented a model, based on the receptor potential nonlinearities of single hair cells. With their model they could account for the occurrence of notches and phase changes in DPOAE $1 / O$ curves. This model seems applicable to the generation of DPOAE I/O curves especially from the frog's BP. Not only are all nerve fibers tuned to a single frequency in this papilla, the absence of traveling waves, and the 
mipolarity of all hair burdles effectwely reduce the entire papilla to a "single hair cell". The model of Lukashkin and Russell always gives a change in phase of exactly $\pi$ rad across the kneepoint/notel in amplitude data. The phase data presented here, both for AP-DPOAE and BP-DPOAE showed phase shifts between 0 (no phase shift) and $2 \pi \mathrm{rad}$. This result excludes this type of model to be applicable to DPOAE generation in the amphibian inner eat.

The third possible explanation involves the interference between two discrete DPOAt components (Whitehead et al, 1992, Manley et al., 1993). One component is assumed to be dominant for high stimulus levels. The other component dominates for low stimulus levels, but saturates for higher stimulus levels. The amplitude and phase curves from the amphibian ear all show a transition from low- to highlevel stimuli. Consequently, our data are consistent with a description of DPOAEs originating from two distinct sources, as has been proposed for marmals.

A recent model used to simulate DPOAF $1 / O$ curves from the cochlea postulates that the two DPOAE sources are at different tonotopic locations in the cochlea (Mills, 1.997). The low-level component arises around the place where the stimulns tones restlt in maximum deflection of the basilar membrane, while the high-level component originates more basally in the cochlea. This model can successfully predict several features of DPOAE I/O curves reconded from small mammals. Since the AP exhibits tonotopic organization, a similar model may be applicable to the AP, although a traveling wave has not yet been identified. In contrast, the BP lacks tonotopic organization and essentially functions as a single auditory filter (Ronken, 1991). Nevertheless, it may be that a two-source model is applicable to the BP, with each source in a different functional/anatomical structure of the papilla.

\subsubsection{Slopes of DPOAE I/O curves}

For high-level stimulus tones, DPOAE $1 / 0$ curves from both papillae in the frog show similar growth characteristics. On the other hand, for low-level stimulus tones there was a conspicuous difference between the slopes of DPOAE I/O curves from the AP and the BP. We will separately discuss the properties of AP- and BP-DPOAE $1 / O$ curves, starting with the AP. Parallel with this, comparisons with DPOAE I/O curves recorded in various other vertebrates will be made.

The slope of DPOAE I/O curves in mammals, birds and reptiles depends on the level of the stimulus tones. For low stimulus levels the growth is usually shallow [1. $\mathrm{dB} / \mathrm{dB}$ or less] (see Table 2.1 for an overview and references). In some species the slope shows a dependency on frequency (humans; Lonsbury-Martin et al., 1990, birds; Kettembeil et al., 1995), but for example in rabbits (Whitehead et al., 1992), this trend is absent. In contrast, for higher stimulus levels DPOAE growth is steep, 


\begin{tabular}{|c|c|c|}
\hline & $\leq 1 \mathrm{~dB} / \mathrm{dB}$ & $>1 \mathrm{~dB} / \mathrm{dB}$ \\
\hline High level slope & none & $\begin{array}{l}\text { All species investigated, ine. } \\
\text { Frog } \mathrm{AP}+\mathrm{BP}[1 \text {, this work] } \\
\text { Alligator lizard [2] } \\
\text { Bobtail lizard }[3] \\
\text { Chicken }[4,5,6] \\
\text { Starling }[5] \\
\text { Mammals }[7,8,9,10,11,12 \text {, } \\
\qquad 13,14,15,16]\end{array}$ \\
\hline Low level slope & $\begin{array}{l}\text { Frog AP }[1, \text { this work } \\
\text { Bobtail lizard }[3] \\
\text { Chicken }[4,5,6] \\
\text { Starling }[5] \\
\text { Mammals }[7,8,9,10,11,12, \\
\qquad 13,14,15,16]\end{array}$ & $\begin{array}{l}\text { Frog BP [1, this work] } \\
\text { Aligator lizard [2] }\end{array}$ \\
\hline
\end{tabular}

Table 2.1: Slopes of DPOAE 1/O curves as reported in literature for several species from the phylum Chordata (all classes except Pisces). The slopes reported are for the distortion product at $2 f_{1}-f_{2}$, and for DPOAE $1 / 0$ curves where both primary tone levels were simultaneously varied. Due to the use of various ways of determining the slopes, as well as the various anount of detail (e.g. frequency dependency) in the different references, we simplified by only reporting the slopes with reference to a slope of $1 \mathrm{~dB} / \mathrm{dB}$. For a slope $<1$ $\mathrm{dB} / \mathrm{dB}$, the underlying nonlinear system is compressive. A slope $>1 \mathrm{~dB} / \mathrm{dB}$ corresponds to an expansive nonlinearity. Not all references explicitly state the slopes of DPOAE I/O curves. In those cases we estimated the slope from the figures presented. References: [1] Van Dijk et al. (2003); [2] Rosowski et al. (1984); [3] Manley et al. (1993); [4] Froymovich et al. (1995); [5] Kettembeil at al. (1995); [6] Sun et at. (2000); [7] Abdala (2000); [8] Brown (1987); [9] Dorn et al. (2001); [10] Dreisbach and Siegel (2001); [11] Gaskill and Brown (1990); $\mid 12]$ Kim (1980); [13] Lonsbury-Martin et al. (1987); [14] Lonsbury-Mantin el al. (1990); [15] Mills and Rubel (1996): [16] Whitehead et al. (1992).

and usually much larger than $1 \mathrm{~dB} / \mathrm{AB}$. Our results show that DPOAE growth in the frog's amphibian papilla is similar to that in mammals, birds and one reptile.

As discussed above, a possible explanation for the presence of kneepoints/notches in DPOAE I/O curves is the involvement of two generation mechanisms. As we will. argue, one may be passive, the other active. Here, an active mechanism refers to a system for which the signal energy in the output does not originate from the input signal alone. For example, in the vertebrate hair cell, the transduction current is controlled by transduction channel opening, but a substantial part of the energy derives from the electrical potential gradient across the channel. "Thus, forward 
transduction is evidently active.

For a biological system, active processes rely on metabolism. Dismption of the healthy physiological condition of the systen will deteriorate active mechanisms. Across species, it has been shown that the low-level mechanism is more vulnerable to acoustic trama, anoxia and phamacological agents than the mechanism dominant at high stimulus levels (mammals: Zurek et al., 1982; reptiles: Manley et al., 1993; birds: Sun et at, 2000; amphibia: Van Dijk et al., 2003). This differential wumerability has been interpreted as being evidence for the involvement of an active mechanism in the generation of low-level DPOAE, while the mechanism involved in the generation of high-level DPOAE may originate from the passive nonlinear nesponse of the inner ear.

In marnmals, somatic outer hair motility (Brownell et al., 1985) has been proposed to be this active mechanism. It has been shown that this somatic motility relies on the presence of the protein prestin in the hair cell basolateral membrane (Zheng et al., 2000), and its effect on DPOAE tresholds shows that it is a critically important component of the mammalian cochlear amplifier (Liberman et al., 2002). In amphibians, and other nonmammalian vertebrates, there is no evidence of somatic motility of the hair cells (He et al., 2003; Manley, 2001).

An alternative amplifier may be the active movement of hair bundles. This movement has been shown in vitro in both nommammalian vestibular systems (Hudspeth, 1997) and the hearing organ of turtles (Crawford and Fettiplace, 1985). In wivo evidence for active movement of hair bundles has been presented for the bobtail lizard (Manley, 2002). Possibly, active hair bundle movement also occurs in mammalian outer hair cells (Kennedy et al., 2003). Hence, it is conceivable that active hair bundle movement might be an active mechanism in mammals and nonmammals. In mammals this may act in parallel with somatic motility of outer hair cells.

In the BP the properties of DPOAE $1 / 0$ curves were markedly different from those in the AP (Fig. 2.9). The slopes of BP-DPOAE I/O curves evoked with both low and high stimulus levels was greater than $1 \mathrm{~dB} / \mathrm{dB}$. This is at variance from all other vertebrates investigated except the alligator lizard (component 1 and 2 in Rosowski et al., 1984; Table 2.1). Both in the frog BP and in the alligator lizard, the slopes of the growth functions are similar for low and high stimulus levels.

Two observations suggest that the frog basilar papilla may be a passive anditory receiver: [1] DPOAEs from the BP are less sensitive to physiological trauma. than DPOAEs from the AP (Van Dijk et al, 2003), and [2] the apparent absence of spontaneous otoacoustic emissions from the BP (Van Dijk and Manley, 2001). Possibly, the steep growth of DPOAEs, as found in the AP at high stimulus levels and in the BP at both low and high stimulus levels, is a signature of passive DPOAE generation. But how can BP-DPOAE be passively generated, if the transduction 
mechanism is active? Possibly, BP-DPOAE arise from the nonlinear stiffness of hair bundles. Although this nonlinear stiffness involves the transduction channel (Howard and Hudspeth, 1988), it does not rely on the active forward transduction current. Thus, DPOAEs from the the BP may be generated passively.

On the other hand, in the alligator lizard spontaneous otoacoustic emissions have been found (Manley, 2000) over the same frequency range for which the DPOAF $1 / O$ curves show steep slopes at both low and high stimulus levels. Thus, although the frog $\mathrm{BP}$ and the alligator lizard take a special position with respect to their DPOAE properties, the implications of this are not yet clear. The phase change associated with the transition from low to high stimulus levels systematically increased with increasing frequency (Fig. 2.10). This suggests that at low stimulus levels the effective dynamic order of the basilar papilla is larger than for high stimulus levels. Possibly, with the saturation of the low-level DPOAE at intermediate stimulus levels, some dynamic orders saturate, and the BP effectively becomes a lower-order system.

\subsubsection{Conclusion}

In the present paper we have shown that, based on their slopes, DPOAE I/O curves from the amphibian ear can be divided into two segments: one for low stimulus levels and one for high stimulus levels. The transition between these two segments in the amplitude data is accompanied by a phase change. A model based on the receptor potential nomlinearities of single hair cells (Lukashkin and Russell, 1998) can not explain the observed emission behavior. Also, the shape of the DPOAE I/O curves cannot be accounted for by assuming a transition in the mode of vibration of the inner ear structures. Rather, our findings are in agreement with the hypothesis that DPOAE I/O curves are the composite of two components, each of which is dominant for a different range of stimulus levels.

For low-level stimulus tones, the slopes of $1 / O$ curves showed a conspicuous difference between AP-DPOAE and BP-DPOAE. This difference in slopes suggest important differences between DPOAE mechanisms in the AP and the BP. Based on the observations that the generation of spontaneous otoacoustic emissions is limited to the AP (Van Dijk and Manley, 2001) and that low-level AP-DPOAE are more vulnerable to inner ear insults than low-level BP-DPOAE (Van Dijk et al., 2003) we hypothesize that in the amphibian papilla the generation of the low-level DPOAE component involves an active mechanism. In contrast, for the basilar papilla we suggest that DPOAEs originate from passive nonlinearities. 


\subsection{Acknowledgements}

This work was supported by grants from the Netherlands Organization for Scientific Research (NWO), and the Heirsius Houbolt: Foundation. 
Chapter 3

\title{
Characteristics of distortion product otoacoustic emissions in the frog from $L_{1}, L_{2}$-maps
}

\begin{abstract}
For a given set of stimulus frequencies $\left(f_{1}, f_{2}\right)$, the level of distortion product otoacoustic emissions (DPOAEs) varies with the levels of the stimulus tones. By systematic variation of the stimulus levels, $L_{1}, L_{2}$-maps of DPOAE amplitude and phase can be constructed. In mammals, these maps suggest that DPOAEs originate from two level-dependent components. Here, we report on $L_{1}, L_{2}$-maps for DPOAEs from the frog ear. In general, these maps were similar to those obtained from the mammalian cochlea. There was a conspicuous difference between the equal-level contourlines for low-level and high-level DPOAEs, which could be modeled by a saturating and an expansive nonlinearity, respectively. The transition from the high-level to the low-level response was accompanied by a DPOAE phase change, which varied systematically between 0 and $\pi$ rad with increasing stimulus frequency. These results suggest that in the frog low-level and high-level DPOAEs are generated by separate nonlinear mechanisms.
\end{abstract}




\subsection{Introduction}

Otoacoustic emissilons (OAEs) are weak sounds that can be measured in the ear canal, both with and without the presence of an extermal stimulus. When the stimulus consists of two pure tones, with appropriately chosen frequencies $\left(f_{1}, f_{2}\right)$ and amplitudes $\left(L_{1}, L_{2}\right)$, so-called distortion product OAEs (DPOAEs) can be recorded at various intermodulation frequencies of the two stimulus tones $\left(e \cdot g .2 f_{1}-f_{2}\right.$ and $\left.2 f_{2}-f_{1}\right)$. To date, most studies of DPOAEs have been on mammals, for which it is generally assumed that (at least for low-level stimuli) electromotility of the outer hair cells is the responsible mechanism for OAE generation (Brownell et al. 1985). Assuch, DPOAEs provide a noninvasive tool to investigate the (nonlinear) mechanics of hair cells that are involved in the transduction of sound.

When, for a given combination of sitimulus frequencies, the levels of the two stimulus tones are varied, the input/output (I/O) characteristics of DPOAEs may be determined. In such experiments typically both stimulus levels are varied, while kesping the difference $L_{1}-L_{2}$ fxed. The resulting I/O-curves exhibit a complex shape, with the rate of DPOAE-aruplitude growth dependent on absolute stimulus levels (mammals: Brown, 1987; birds: Froymovich et al., 1995; reptiles: Manley et al. 1993; amphibians: Meenderink and Van Dijk, 2004). One way to explain the shape of these $1 / O$-curves (and associated DPOAE-phase) is by interference of two level-dependent components, of which ach is dominant over a different range of stimulus levels (Whitehead et al, 1992). The different vulnerabillity of these components has led to the suggestion that they arise from different mechanisms. The low-level component presumably arises from "active" (i.e. energy is supplied by the ear in such manner that sound-induced vibration is enhanced) hair cell mechanics, whille the component that dominates for high-level stimuli is generated by some "passive" (2.e. no energy is supplied by the ear) nonlinearity, that is not necessarily associated with hair cells (Mills et ak, 1993).

Recently, doubt has risen whether such a two-source model is necessary to account for DPOAE-generation in the mammalian cochlea (Mills, 2002). It seens that the division into a passive high-level and an active low-level component is no longer supported (Mom et al., 2001; Frolenkov et al, 1998). The results from these and other studies in mammals indicate that the generation of low-level and high-level DPOAEs both involve an active nonlinear mechanism that critically depends on the nomal functioning of the outer hair cells. In conjunction, several $1 / O$-characteristics ( 2 e. differential vulnerability and notches) can be accounted for by models that incorporate only a single, active mechanism for DPOAE-generation (Mills, 1997; Lukashkin et ol., 2002). Seemingly, only one type of nonlinear mechanism is necessary for DPOAE-generation. However, mice lacking the protein prestin in their 
hair cell's basolateral membranes do generate high-level DPOAEs (Liberman et al., 2004). Apparently, in contrast to low-level DPOAEs in mice, high-level DPOAEs do not depend on prestin motility and thus must originate from another mechanism.

In frogs, the anatomy of the inner ear deviates considerably from that found in mammals, the most prominent difference being the absence of a cochlea. Instead, the frog inner ear contains two papillae that respond to airborne sound. Of these, the amphibian papilla (AP) shows several similarities with the cochlea. It consists of an elongated strip of sensory epithelium that exhibits tonotopic organization (Lewis et al., 1982). As in the cochlea, the entire epithelium is covered by a tectorial membrane. Hair cells are innervated by both efferent and afferent nerve tibers, the latter having tuning characteristics similar to those found in mammals (Lewis, 1992). The AP may generate both spontaneous and distortion product OAEs (Van Dijk and Manley, 2001). Different from the cochlea are the absence of a basilar membrane and of distinct groups of hair cells (i.e no imner and outer hair cells).

The structure of the other papilla, the basilar papilla (BP), is much simpler. It consists of a small patch of sensory epithelium, covered by a tectorial membrane. In R. catesbeiana, the entire papilla contains between 50 and 100 hair cells whose stereovillar bundles are oriented identically (Lewis, 1978). As in the AP, this papilla lacks a basilar membrane and hair cells are not differentiated into differing groups. The BP lacks tonotopic organization; in individual frogs, the majority of afferent nerve fibers is tuned to a single frequency (Ronken, 1991; Van Dijk et. al., 1997). These properties ensure that the entire papilla essentially functions as a single auditory filter (Ronken, 1990; Van Dijk and Manley, 2001). The BP may only generate distortion product OAEs; no spontaneous OAEs have been reported from this papilla.

Recently, we presented $1 / O$-curves for DPOAEs from the frog ear that are very similar to I/O-curves obtained in other vertebrates (Meenderink and Van Dijk, 2004). We suggested that DPOAEs in the frog are the resultifrom two level-dependent components, with each component arising from a different nonlinear mechanism. Here, we further detail the stimulus-level dependence of DPOAEs in the frog by presenting full $L_{1}, L_{2}$-maps. These maps showed a differential dependence of highlevel and low-level DPOAEs, as is evidenced by the different contourlines. Combined with the observed frequency-dependent phase change associated with the transition from low-level to high-level DPOAEs, these results provide further support for the involvement of two separate, level-dependent emission-generation mechanisms in the frog. 


\subsection{Materials}

Distortion product otodcoustic emissions were recorded from 12 ears of 7 Northern leopard frogs, Rana pipiens pipiens; bady mass: $37.6-46.8 \mathrm{~g}$ (mean: $42.7 \mathrm{~g}$ ). In order to immobilize the frog during DPOAE recording, it was anesthetized with an intramuscular injection of pentobarbital sodium solution (diluted Nembutal: 6.0 $\mathrm{mg} / \mathrm{ml} ; 10 \mu \mathrm{l} / \mathrm{g}$ body weight) in one of the hind limbs. Approximately 25 minutes after the administration of the anesthetic agent, the frog was transferred to a soundattenuating chamber and placed between wet gauze to prevent dehydration and to facilitate cutaneous respiration.

A custom-built probe assembly was carefully placed with its open end against the skin surrounding the tympanic membrane. A tight seal between the probe and the skin was obtained using vaselin. The probe assembly contained two miniature speakers (E-A-RTONE 3A, Aero Company, IN, USA) for stimulus delivery, and a 1" high-sensitivity condenser microphone (B\&KK type 4179, Brüel \& Kjær, Denmark) for emission recording. DPOAEs were evoked by stimulating the ear with two simultaneously played pure tones. These stimulus tones, with frequencies $f_{1}$ and $f_{2}$ $\left(f_{2}>f_{1}\right)$, were generated from two separate D/A channels (RP2, Tucker-Davis Technologies, Gainesville, FL, USA), attenuated in order to set their levels (PA5, TDT, Gainesville, FL, USA) and each one was played through a separate miniature speaker.

The microphone signal was passed through a low-noise amplifier (B\&K type 2660; Brüel \& Kjær, Denmark; amplification $=20 \mathrm{~dB}$ ) and a measuring amplifier (B\&K type 2610; Brïel \& Kjæer, Denmark; amplification $\geq 0$ dB). This amplified microphone signal was recorded using an A/D channel (RP2, TDT, Gainesville, FL, USA) and stored on computer disc for offline analysis. In order to monitor the DPOAEs during the experiment, the frequency spectrum of the amplified microphone signal was viewed online using a spectrum analyzer (SR760, Stanford Research Systems, Sunnyvale, CA, USA). Customized software, written in MATLAB (The Mathworks Inc, Natick, MA, USA) and RPvds (TDT, Gainesville, FL, USA) was used to control stimulus tone generation and signal recording. The recorded signal was averaged in the time-domain after the application of a level-crossing artifact-rejection method. From the averaged signal, both the DPOAEs and the noise floor were calculated using Fourier analysis. For further details concerning data acquisition and analysis we refer to Meenderink and Van Dijk (2004).

Data were collected at various different stimulus frequency combinations (overall range: $313<f_{1}<3213 \mathrm{~Hz} ; f_{2} / f_{1}=1.02,1.1$ or 1.3 ). For each $f_{1}, f_{2}$ combination, stimulus levels were independently and randomly varied over a $30 \mathrm{~dB}$ range in 3 $\mathrm{dB}$ steps. This resulted in a square $L_{11}, L_{2}$ matrix containing 121 points for each 

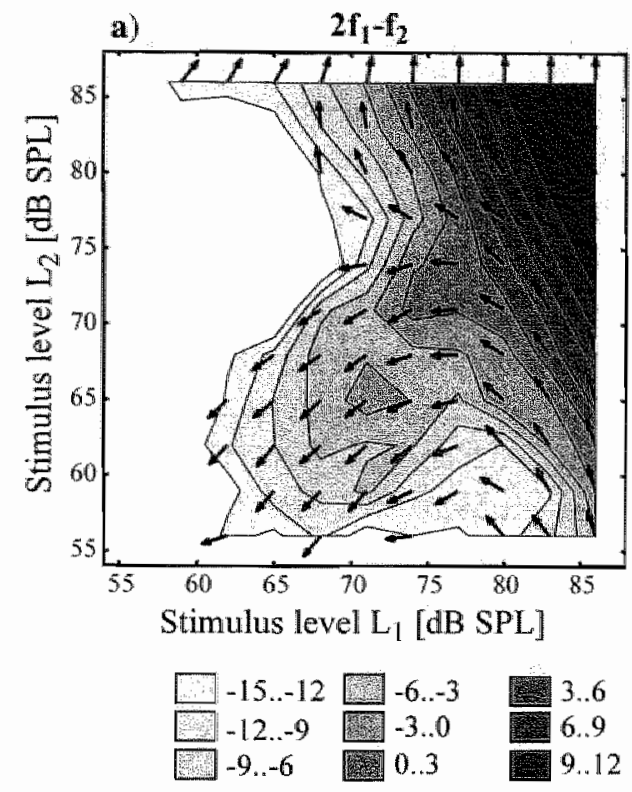
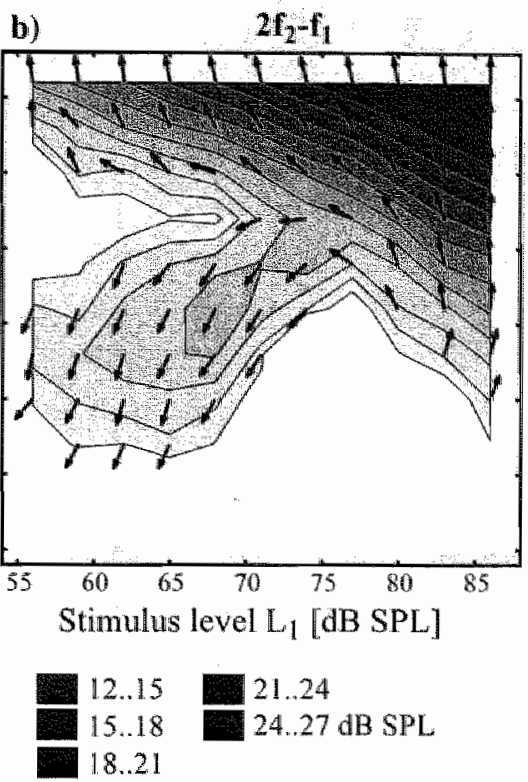

Figure 3.1: Amplitude and phase of DPOAE at (a) $2 f_{1}-f_{2}$ and (b) $2 f_{2}-f_{1}$ as function of stimulus levels $L_{1}$ and $L_{2}$. Shown are data recorded in one frog for $f_{1}=1913 \mathrm{~Hz}$ and $f_{2} / f_{1}=1.02$. This combination of stimulus frequencies results in DPOAEs originating from the frog basilar papilla. DPOAE amplitude is represented by the equal-amplitude contourlines. The lowest contourlines are at $-15 \mathrm{~dB}$ SPL and subsequent contonlines are drawn in $3 \mathrm{~dB}$ increments. The consecutive intervals between the contourlines are coded in gray, as given in the key. Phase of the DPOAE is given by the arrows overlaying the contourlines. The phase is relative to the phase at $L_{1}=L_{2}=86 \mathrm{~dB}$ SPL.

stimulus frequency combination.

To determine the distortion of the recording system, additional experiments were performed with the open end of the probe assembly closed by a solid surface. In general, system distortion was approximately 90 dB below the stimulus levels. Only for low stimulus level combinations did the system distortion disappear in the noise floor. Based on these recordings, only DPOAEs for which the armplitude exceeded -15 dB SPL were arranged in $L_{1}, L_{2}$ matrices. Since the phase of the two stimulins tones was not controlled in the experiments, the DPOAE phase will be given relative to the phase of the two stimulus tones according to: $\Phi_{n f_{1}-m f_{2}}=\phi_{n f_{1}-m f_{2}}-\left(n * \phi_{1}-m * \phi_{2}\right)$, where $\phi_{m f_{3}-m f_{2}}$ is the recorded DPOAE phase, and $\phi_{1}$ and $\phi_{2}$ are the calculated phases of the two stimulus tones.

All procedures were approved by the animal experiment committee of the Maastricht University. 


\subsection{Results}

Distortion product oboaconstic enissions (DPOAEs) could be detected in all ears investigated, Although for certain stimulus tone combinations, DPOAES cond be observed at up to 8 different distortion frequencies, here only DPOAEs at frequencies $2 f_{1}-f_{2}$ and $2 f_{2}-f_{1}$ will be presented. The DPOAE data were visualized by plotting both the amplitude and the phase of the DPOAE against the levels of both stimulus tones. With such an $L_{1}, L_{2}$ map, contourlines of equal emission amplitude were calculated at $3 \mathrm{~dB}$ intervals. Only data points with amplitudes $>-15 \mathrm{~dB}$ SPL were included. Phase was represented by arrows, with the phase for the largest $L_{1}, L_{2}$ combination arbitrarily set to zero. As such, absolute phase holds no meaning, but phase changes within each $L_{1}, L_{2}$-map do.

Fig. 3.1 displays results for DPOAEs at $2 f_{1}-f_{2}$ (Fig. 3.1a) and $2 f_{2}-f_{1}$ (Fig. 3.1b) obtained from the frog basilar papilla $\left(f_{1}=1913 \mathrm{~Hz}, f_{2} / f_{1}=1.02\right.$ with $L_{1}$ and $L_{2}$ between 56 and $86 \mathrm{~dB} \mathrm{SPL})$. In this figure, the $3 \mathrm{~dB}$ intervals between consecutive amplitude contourlines are coded in gray, with the corresponding DPOAE amplitudes given in the key. Focussing on the amplitude, each $L_{1}, L_{2}$-map could be divided into two principal regions based on the shape of the contourhnes. For relatively low stimulus levels, the contourlines were clearly convex. As a result, these lines encompass a more or less pronounced ridge in the $L_{1}, L_{2}$-map which lies approximately parallel to the diagonal $L_{1}=L_{2}$. In contrast, when one or both of the stimulus levels were relatively high, the contourlines approximated straight lines, with consecutive contourlines being parallel to each other. The slope of these contourlines depended on the DPOAE frequency: $L_{2} / L_{1}=-2 \mathrm{~dB} / \mathrm{dB}$ for $2 f_{1}-f_{2}$ and $L_{2} / L_{1}=-0.5$ $\mathrm{dB} / \mathrm{dB}$ for $2 f_{2}-f_{1}$.

Figure 3.2: (opposite page) Overview of the stimulus level dependence of DPOAE amplitude and phase for different stimulus frequencies. All data were obtained in a single ear using $f_{2} / f_{1}=1.1$. The left column gives $\mathrm{DPOAE}$ at $2 f_{1}-f_{2}$, while the right column gives DPOAE at $2 f_{2}-f_{1}$. (a-b) DPOAE amplitude as function of stimulus frequency $f_{1}$ obtained with $L_{1}=L_{2}=80 \mathrm{~dB}$ SPL. The vertical dashed lines indicate the frequencies $\left(f_{1}=814,1052,1507,1907 \mathrm{~Hz}\right)$ for which $L_{1}, L_{2}$ recordings were made. The labels on the dashed lines refer to the subplots in the figure. (c-j) Amplitude and phase of DPOAE as function of stimulus levels $L_{1}$ and $L_{2}$. The lay-out of the plots is similar to that in Fig. 3.1. That is, DPOAE smplitude is represented by equal-intensity contourlines, drawn at $3 \mathrm{~dB}$ intervals. DPOAE amplitude is represented by shadings of grey, with the corresponding levels given in the key. DPOAE phase is represented by the arrows overlying the contourlines. In each subplot, phase is relative to the phase at $L_{1}=L_{2}=86 \mathrm{~dB}$ SPL. As such, phase differences between the different subplots hold no meaning, but changes in plase within each subplot do. 

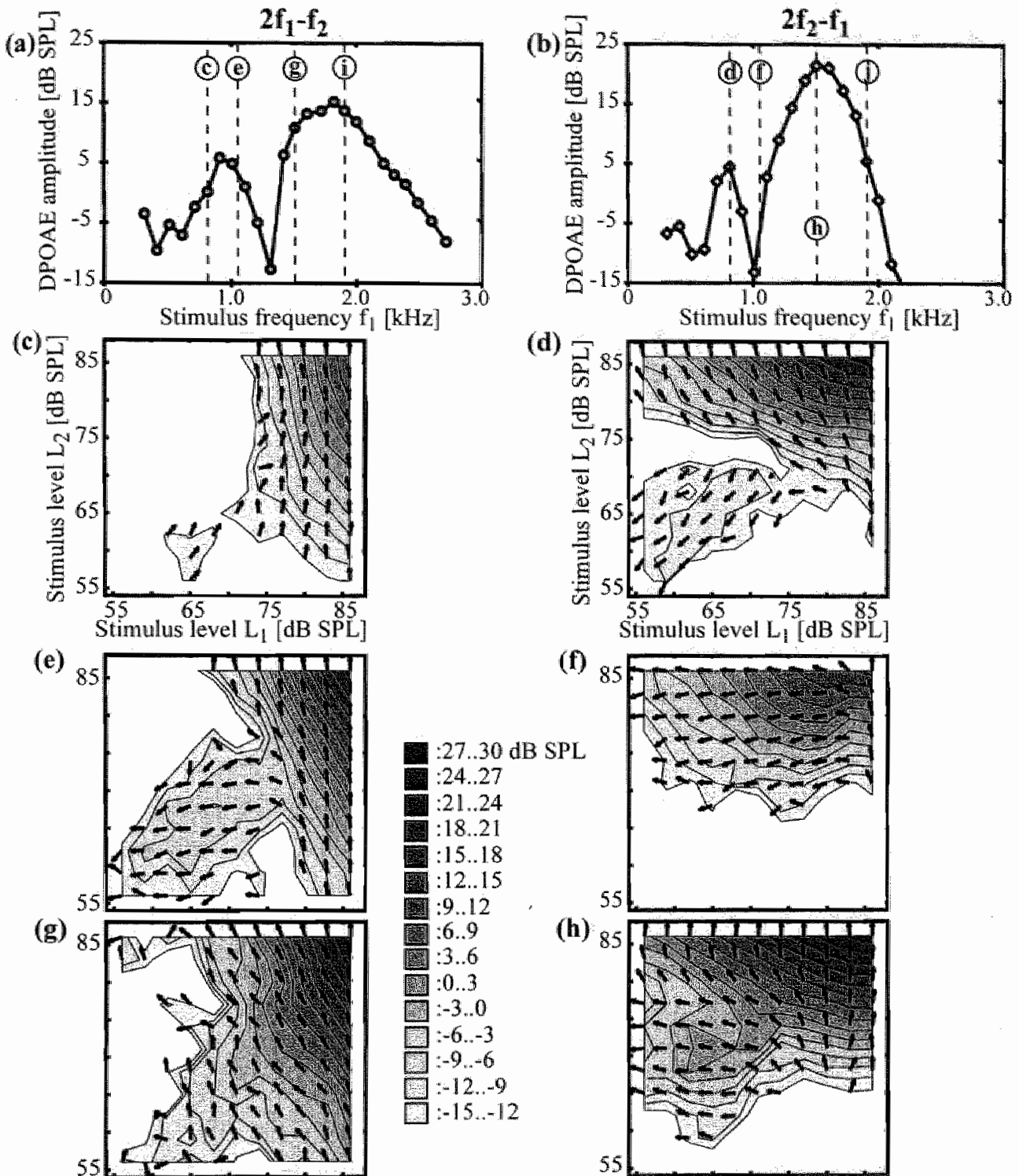

(h)

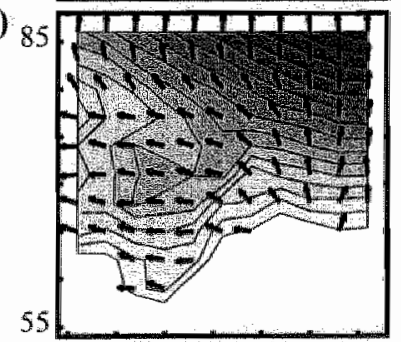

(i)

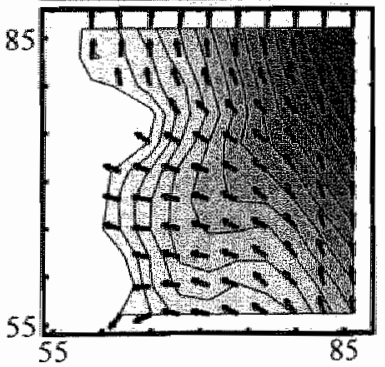

(j)

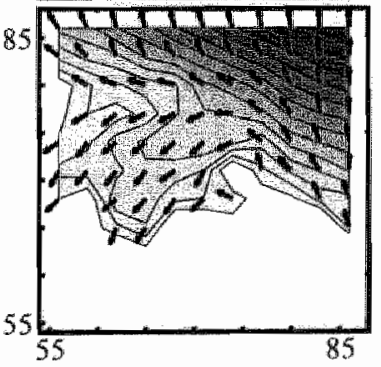


For DPOAE phase, the $L_{1}, L_{2}$-maps could be divided into the same two principal regions as the amplitude data ( $i$.e. wo relatively low-level stimuli versus at least one relatively high-lovel stimulus). Within each of these two regions phase was relatively constant, while betwen them the phase was different. In the examples shown, this phase difference is approximately $0.7 \pi \mathrm{rad}$ for $2 f_{1}-f_{2}$ (Fig. 3.1a) and $0.9 \pi$ rad for $2 f_{2}-f_{1}$ (Fig. 3.1b). For clarity, we will refer to the two principal regions in the $L_{1 .} L_{2}$-naps as "low-level" (relatively low stimulus levels) and "high-level" (at least one of the two stimulus levels is relatively high), respectively.

In frogs, DPOAEs arise from either one of the two hearing papillae, depending on the stimulus frequencies used (Van Dijk and Manley, 2001; Meenderink and Van Dijk, 2004). This is reflected in the bimodal shape of the DPOAE-audiograms, as illustrated in Figs. 3.2a,b. DPOAEs evoked with low-frequency stimulus tones $\left(f_{1}<1250 \mathrm{~Hz}\right.$ for $2 f_{1}-f_{2}$ and $f_{1}<1000 \mathrm{~Hz}$ for $2 f_{2}-f_{1}$, respectively) arise from the amphibian papilla, while DPOAEs evoked with larger stimulus frequencies originate from the frog basilar papilla. As an overview, the remaining subplots in Fig. 3.2 show $L_{1}, L_{2}$-maps at 4 stimulus frequency combinations, all obtained in one ear. For reference, the stimulus frequencies $f_{1}$ for which these $L_{1}, L_{2}$-maps were recorded are illustrated by the vertical dashed lines in Figs. $3.2 a_{y} \mathrm{~b}$.

These $L_{1}, L_{2}$-maps showed a similar dependence of DPOAEs on stimulus levels as was described for Fig. 3.1: [1] for all stimulus frequencies, there was a distinction between the stimulus level dependence of low-level and high-level DPOAEs. This is illustrated by the convex shape of equal-amplitude contourlines for the low-level emissions, and the approximately straight contourlines for high-level emissions. [2] The low-level ridge, encompassed by the convex contourlines, ran approximately parallel to the diagonal $L_{1}=L_{2}$ for both $2 f_{1}-f_{2}$ and $2 f_{2}-f_{1}$. [3] The straight high level contourlines had slopes equal to $-2 \mathrm{~dB} / \mathrm{dB}$ for $2 f_{1}-f_{2}$ and $-0.5 \mathrm{~dB} / \mathrm{dB}$ for $2 f_{2}-f_{1}$. [4] Within the low- and the high-level region of the $L_{1}, L_{2}$-maps, DPOAE phase was relatively constant, but the transition between both regions was accompanied by a change in phase. Whthin each papilla, this change increased from close to 0 rad for low frequencies to almost $\pi$ rad for high frequencies (see Fig. 3.3 ).

Fig. 3.2 also shows that there was a clear distinction between DPOAEs from the amphibian papilia and the basilar papilla. In the AP, low-level emissions resulted in an extensive plateau in the $L_{1}, L_{2}$-maps. In contrast, for DPOAEs in the BP this low-level plateau was much smaller or even absent.

The low-level ridge was not always on the diagonal $L_{1}=L_{2}$, but its position depends on the stimulus frequency relative to the peak frequency of the DPOAEaudiogram. This can be illustrated most clearly for emission measurements at relatively large stimulus frequency ratios. Fig. 3.4 displays results for one subject 


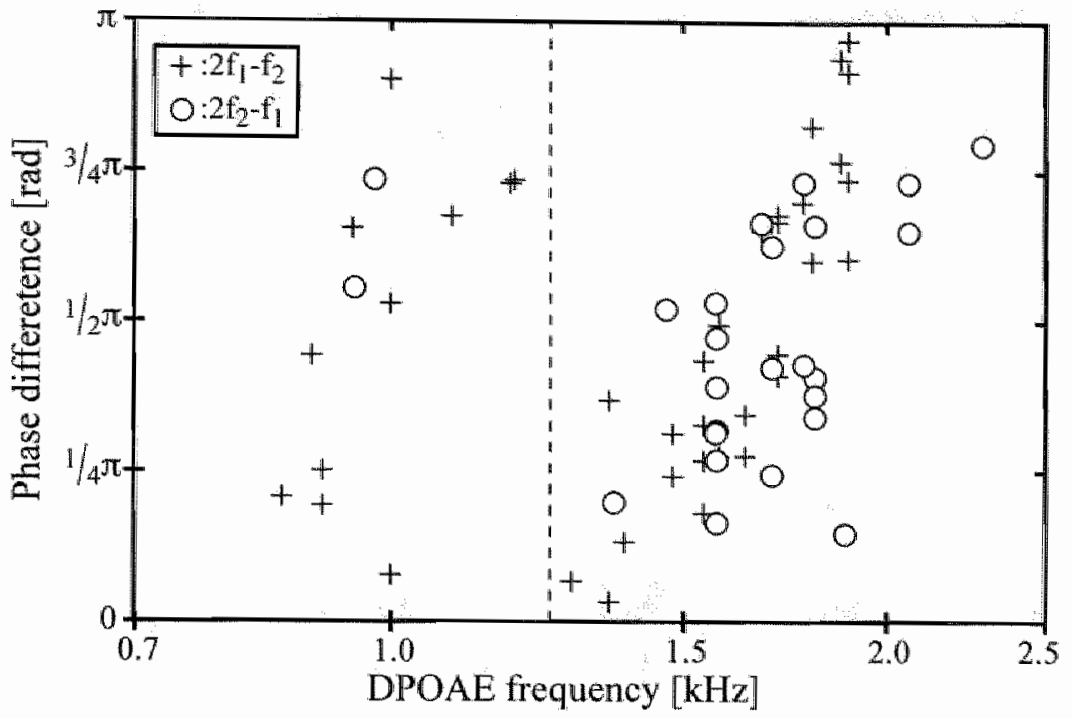

Figure 3.3: Difference in phase for DPOAE at $2 f_{1}-f_{2}(+)$ and $2 f_{2}-f_{1}$ (O) evoked with $L_{1}=L_{2}=86$ and $L_{1}=L_{2}=63 \mathrm{~dB}$ SPL as a function of distortion product frequency. Data obtained with $f_{2} / f_{1}=1.02$ and 1.10 are combined. The vertical dashed line gives the frequency separation between the amphibian papilla and the basilar papilla (1250 $\mathrm{H} z$; Ronken., 1991).

when $f_{2} / f_{1}=1.30$. At this ratio, only DPOAEs from the BP at $2 f_{2}-f_{1}$ had an amplitude which was sufficient for systematic evaluation. The DPOAE-andiogram contains a single maximum at $f_{1}=1313 \mathrm{~Hz}$ (Fig. 3.4a). For a stimulus frequency $f_{1}$ at this maximum, the low-level ridge in the $L_{1}, L_{2}$-map is approximately on the diagonal $L_{1}=L_{2}$ (Fig. 3.4c). However, when this stimulus frequency was on the low-frequency slope of the DPOAE-audiogram, $L_{1}$ needed to be larger that $L_{2}$ to obtain a maximum emission amplitude. Consequently, the low-level ridge in the $L_{1}: L_{2}$-map is below the diagonal (Fig. $3.4 \mathrm{~b}$ ). For a stimulus frequency above the maximum in the DPOAE-audiogram, the reverse was true: $L_{2}$ needed to be larger than $L_{1}$ for a maximum emission amplitude, and the low-levels ridge lies above the diagonal (Fig. 3.4d). Nevertheless, for each stimulus frequency combination, the low-level ridge was parallel to the main diagonal $L_{1}=L_{2}$.

\subsection{Discussion}

It is currently believed that distortion product otoacoustic emissions (DPOAEs) from the frog arise from both the amphibian papilla (AP) and the basilar papilla (BP), the two end. organs in the frog inner ear that are most sensitive to airborne sound. 

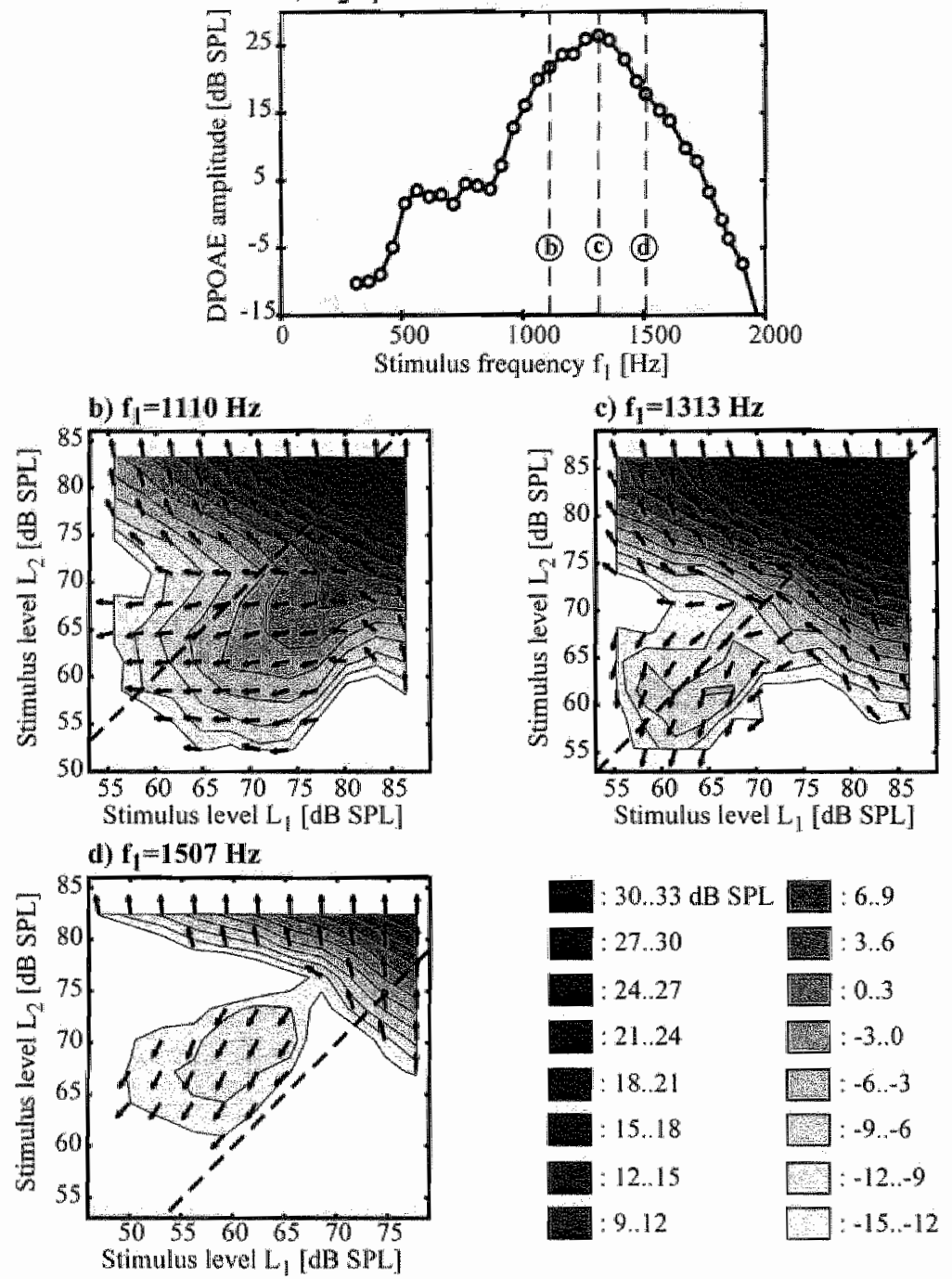

Figure 3.4: DPOAEs at $2 f_{2}-f_{1}$ from the basilar papilla for $f_{2} / f_{1}=1.30$. (a) DPOAE amplitude function of stimulus frequency $f_{1}$ obtained with $L_{1}=L_{2}=80 \mathrm{~dB} \mathrm{SPL}$. The vertical dashed lines give the frequencies $\left(f_{1}=1110,1313,1507 \mathrm{~Hz}\right)$ for which $L_{1}, L_{2}$ recordings were made. The labels on these dashed lines refer to the subplots in the figure. (b-d) Amplitude and phase of DPOAE as function of stimulus levels $L_{1}$ and $L_{2}$. The diagonal dashed line in each subplot corresponds to $L_{1}=L_{2}$. The position of the low-level ridge relative to this diagonal depends on the stimulus frequency; compare subplots (b), (c) and (d). DPOAE levels are gray-coded in $3 \mathrm{~dB}$ intervals, with the corresponding levels given in the key. DPOAE phase is represented by the arrows overlying the contourlines. 
The bimodal shape of DPOAE-audiograms is a direct result of this (Figs. 3.2a, b; Van Dijk and Manley, 2001; Meenderink et al,, 2005). In the present work we explored the dependence of DPOAE amplitude and phase on stimulus levels by systematically mapping $L_{1}, L_{2}$-space over a range of stimulus trequencies thus covering the frequency range of highest sensitivity of both papillae. The first obvious result is that across frequencies the $L_{1}, L_{2}$-dependence of DPOAEs is very different for high-level emissions compared to low-level emissions. In the presented $L_{1}, L_{2}$-maps, high-level DPOAEs were characterized by contourlines that followed (approximately) straight lines. In contrast, contourlines for low-level DPOAEs were clearly convex (e.g. Fig. 3.1). In mammals, a similar difference between bigh-level and low-level DPOAEs is found (Mills et al., 1993; Mills and Rubel, 1994; Whitehead et al., 1995).

The shape of the contourlines reflects fundamental properties of the nonlinearity that underlies DPOAE generation. For a cubic nonlinear system

$$
y=x^{3}
$$

contourlines are straight (see Figs. $3.5 \mathrm{a}, \mathrm{b}$ ), similar to the high-level DPOAEs in both frogs and mammals. Small deviations from straight contourlines may occur for nonlineairities described by $y=\operatorname{sign}(x)|x|^{\nu}$. For $\nu>3$, contourlines are concave while for $\nu<3(\nu \neq 1)$ contourlines are convex. The slope of the contourlines for a cubic nonlinearity is $L_{2} / L_{1}=-2 \mathrm{~dB} / \mathrm{dB}$ for $2 f_{1}-f_{2}$ and $L_{2} / L_{1}=-0.5 \mathrm{~dB} / \mathrm{dB}$ for $2 f_{2}-f_{1}$, which is similar to the slopes of the high-level contourlines found here (e.g. Fig. 3.1)

If the nonlinearity has a (possible asymptotic) output clipping level, the contourlines have a shape that is markedly different. As an example. Figs. 3.5c,d show the $L_{1}, L_{2}$-dependence of distortion product amplitude for a Boltzman nonlinearity

$$
y=\frac{1}{1+e^{-x}},
$$

which clips to $y=1$ for $x \rightarrow \infty$ and $y=0$ for $x \rightarrow-\infty$. Here, the contourlines are convex, running parallel to the diagonal $L_{1}=L_{2}$ in part of the $L_{1}, L_{2}$-space. Lowlevel DPOAEs from the frog had a dependence on stimulus level which is consistent with a clipping nonlinearity, such as the Boltzman function.

In mammals, low-level contourlines are also convex, but, in general, the low-level ridge is not parallel to the diagonal $L_{\Perp}=L_{2}$ (Whitehead et al., 1992; Kummer et al., 2000; Pibal et al., 2002). Kummer et at. (2000) and others interpreted this as being a manifestation of the differential growth of each stimulus amplitude at the presumed location of DPOAE-generation, which results from the different growth of basilar membrane vibration at different locations along the cochlea (e.g. Ruggero and Rich, 1991). As such, it reflects the transformation of stimulus amplitudes at 

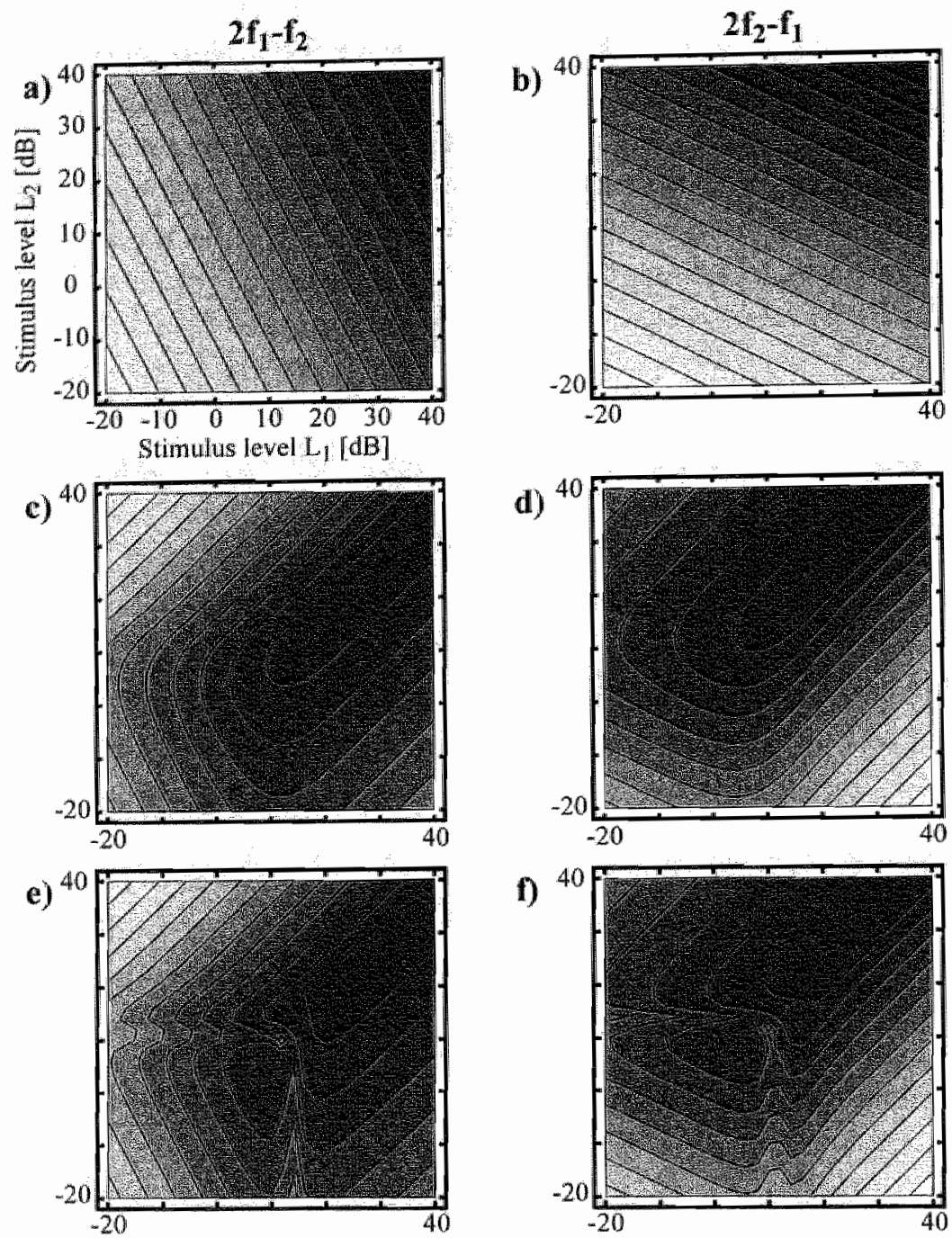

Figure 3.5: $L_{11} L_{2}$-maps for three static nonlinear models. For each model, results are shown for the distortion product at $2 f_{1}-f_{2}$ (left column) and at $2 f_{2}-f_{1}$ (right column). Contourtines were calculated at $10 \mathrm{~dB}$ intervals. a) Amplitude at $2 f_{1}-f_{2}$ for a cubic nonlinearity $y=x^{3}$. b) Anplitude at $2 f_{2}-f_{1}$ for the same nonlinear model c) and d). Results for the Boltzman function $y=1 /(1+\exp (-x))$. e) and $f)$. Results for a Boltzman function with a shifted operation point: $y=\mathbb{1} /(1+\exp (-x+3))$. The combination of the cubic monlinearity (panels a and b) with a Boltzman nonlinearity (panels o and d) may account for the $L_{1}, L_{2}$-maps recorded in the frog. The Boltaman function with a shifted operation point may describe the transition from low- to high-level emissions. However, in that model the high-level contourlines do not match the approximately linear contourlines observed in the frog. 
the tympanic membrane to stimulus amplitudes at the site of DPOAE-generation.

In our results, the low-level ridge was parallel to $L_{1}=L_{2}$ suggesting that the stimulus-induced vibration of the tectorial membranes in both the AP and the BP have the same growth rate. In other words, the tectorial membrane response may grow nonlinearly and compressively, but in contrast to the mechanics in the mammalian cochlea, this growth has to be the same for both stimulus frequencies. Note that the finding that the low-level ridge may be shifter away from $L_{1}=L_{2}$ (Fig. 3.4) indicates that equal stimulus levels at the tympanic membrane do not necessarily result in equal-amplitude vibrations in the tectorial membrane.

Typically, the observed level-dependence of DPOAEs are interpreted as being the result of two level-dependent components (e.g. Kim, 1980; Whitehead et al., 1992). Here, one component is dominant for high-level stimuli, while the second component dominates for low-level stimuli. Based on the differential vulnerability of the two components, it has been suggested that each component arises from a different mechanism (Mills et al., 1993). In this two-source model the low-level component is linked to active hair cell mechanics, while the high-level component arises from a passive nonlinearity, possibly not associated with the hair cells.

Recently, doubt has risen whether the two-source model is applicable to DPOAEgeneration (Mills, 2002). Frolenkov et al. (1998) showed that chemical blocking the outer hair cell motility abolishes both low-level and high-level DPOAEs in the guinea pig, while Mom et al. (2001) provided evidence that the high-level DPOAEs that remain after cochlear ischemia are highly vulnerable to acoustic overstimulation. These results indicate that the high-level and low-level DPOAEs in mammals depend on active mechanisms. Model studies have shown that a properly configured, single Boltzman nonlinearity can account for the nonmonotonicities and associated phase-shifts seen in mammalian DPOAE 1/O data (Lukashkin and Russell, 1998). When such models incorporate feedback they can also reproduce the differential vulnerability of low-level and high-level DPOAEs (Lukashkin et at., 2002). Also, Mills (1997) proposed a model in which one type of active nonlinearity generates DPOAEs. The artificial separation of emissions generated at different locations along the basilar membrane results in the appearance of a "passive" and an "active" component that both arise from the same nonlinearity. In short, the combination of these results imply that both high-level and low-level DPOAEs may arise from one type of active nonlinearity.

However, Liberman et al. (2004) showed that mice lacking the protein prestin in their hair cell's basolateral membrane (i.e. the presumed motor-molecule of the cochlear amplifier is missing) do generate high-level DPOAEs, indicating that hair cell electromotility is not required for their generation. In addition, they found that after death these high-level DPOAEs disappear rapidly, with a time-course 
similar to the post-mortem disappearance of the cochlear microphonic. Therefore, these "residual" high-level DPOAEs were clearly of biological origin and arose from a second active nonlinear mechanism that is perhaps related to the hair cell stereovillar bundles.

In a single Boltzman nonlinear model, a transition from low-to high-level DPOAEs is accomplished by a shift of the operation point of the Boltzman curve (Lukashkin and Russell, 1998). Regardless of the choice of operation point, the Boltaman nonlinearity is a clipper for large stimulus amplitudes. Thus, a Boltzman nonlinearity exhibits a transition from low- to high-levels, but the high-level contourlines are parallel to the main diagonal in the $L_{1}, L_{2}$-maps (See Figs. $3.5 e, f$ ). This is in sharp contrast with our experimental findings (e.g. Figs. 3.1a,b): the frog high-level contourlines were consistent with a cubic-nonlinear function (Eq. 3.1). Thus, we reject a single Boltzman-nonlinearity as a possible model for both low-and high-level DPOAE generation in the frog.

The applicability of the model proposed by Mills (1997) to the frog ear can be excluded a priori. Although it results in similar $L_{1}, L_{2}$-maps as in the frog ear, tonotopy is an essential part of the model. Since the basilar papilla is not tonotopically organized, its general applicability to the frog ear is excluded. Also, the frequency-dependence of the phase shift between the low-level and the high-level DPOAEs (Fig. 3.3) suggests that two nonlinear mechanisms are involved in emission generation. These two mechanisms are somehow coupled by a dynamic element (e.g. a spring or a resistion).

There exist important differences in the growth of low-level DPOAEs from the AP and the mammalian cochlea on the one hand, and from the BP on the other hand. The extensive low-level plateaus in the AP indicate that DPOAE amplitude grows compressively $(<1 \mathrm{~dB} / \mathrm{dB})$ with increasing low-level stimuli, while at low levels growth in the $\mathrm{BP}$ was expansive $(>1 \mathrm{~dB} / \mathrm{dB})$. The Boltzman nonlinearity restults in expansive growth of the low-level distortion-products, in accordance with the expansive growth of low-level DPOAEs in the BP (Meenderink and Van Dijk, 2004 ; this work). The Boltzman nonlinearity has been proposed to describe the relationship between the hair bundle defection and the transduction current in hair cells (Crawford et al., 1989). Thus, DPOAE amplitude in the BP may be proportional to the transduction current in hair cells.

The compressive growth rate in the AP and the mammalian cochlea may be interpreted as being the result of an antomatic gain control in these hearing organs. In other words, the generation of DPOAEs may be proportional to the transduction current in hair cells (described by the Boltzman nonlinearity), but the measured DPOAE-amplitude growth nay reflect the additive effect of a compressive amplifcation mechanism. 
In conclusion, our $L_{1}, L_{2}$-maps support the notion of two DPOAE-generation mechanisms in the frog, one of which dominates for low-level stimuli and a second one which is dominant for high-level stimuli.

\subsection{Acknowledgements}

This work was supported by grants from the Netherlands Organization for Scientific Research (NWO), and the Heinsius Houbolt Foundation. 


\section{The effect of body-temperature on distortion product otoacoustic emissions in the frog}

\section{Abstract}

To study the possible involwement of energy-dependent mechanisms in the transduction of sound within the anuran ear, distortion product otoacoustic emissions (DPOAEs) were recorded in the Northern leopard frog over a range of body-temper atures. We found that the effect of body-temperature depended on the used stimulus levels and on the hearing papilla under investigation. Low-level DPOA fis from the amphibian papilla (AP) were clearly, but reversible, depressed for decreased body temperatures. Apparently, DPOAE generation in the AP depends on metabolic rate. In contrast, in the other hearing organ, the basilar papilla (BP), no clear effect of body-temperature on DPOAEs was found, irrespective of the stimulus levels used. This suggests that this papilla does not rely on a mechanism that consumes energy. The passive functioning of the anuran BP places this hearing organ in a unique position within vertebrate hearing. A passive hearing organ may be beneficial to poikilothermic species, since it will provides the animal with a consistent spectral window, regardless of ambient or body-temperature. 


\subsection{Introduction}

It is currently cominonly accepted that the high sensitivity of the mammalian cochlea finds its origin in a mechanism known as the "cochlear amplifter" (Davis, 1983; Hudspeth, 2989). This mechanism boosts the vibration of the basilar membrane on a cycle-by-cycle basis, thus enhancing the sound-induced movements of this cochlear structure. It is thought that somatic electromotility of outer hair cells is the driving mechanism of this amplifier (Brownell et al., 1985), since the sensitivity of the mammatian cochlea critically depends on it (Liberman et al., 2002).

But outer hair cells are specialized cells found exclusively within the mammalian cochlea, and there is no evidence for somatic motility of hair cells in nonmammalian vertebrates (He ef al., 2003; Manley, 2001). This may suggest that active hearing is reserved for mammals only. However, the observed phenomena that are generally attributed to the cochlear amplifier are found throughout all classes of tetrapod vertebrates (reviewed in Manley (2001)), strongly favoring the idea that active hair cell processes are a universal property of vertebrate hearing. This would require the presence of an alternative "inner ear amplifier" $\Perp$ within the hair cells of nonmammalian vertebrates. A likely candidate is the active movement of hair cell stercovillar bundles which has been shown in several nommammalian vertebrate classes (Crawford and Fettiplace, 1985; Hudspeth, 1997; Manley, 2002). Recent experiments suggest that: this active "hairbundle-mechanism" may also be present in the mammalian cochlea (Kennedy et al., 2003; Liberman et al., 2004), where it may function in conjunction with the somatic motility of outer hair cells.

In frogs, hair cells that are most sensitive to airborne sound are distributed over two anatomically-separated papillae, each one most sensitive to a different range of frequencies. The amphibian papilla (AP), which is most sensitive to the low- and mid-frequencies within the frog hearing range, consists of an elongated strip of hair cells. In contrast to the mammalion cochlea, these cells are directly over the rigid wall of the papillar recess, rather than over a flexible membrane. Overlying the AP hair cells is a tectorial membrane. Both the shape of this membrane, as well as its position within the papillar recess, suggest that it plays an important role in the transduction of sound by hair cells (Lewis and Leverenz, 1983). The papilla exhibits tonotopic organization with the highest $(\approx 1.2 \mathrm{kHz}$ ) and lowest $(\approx 0.1$ kFz) frequencies being represented caudally and rostrally, respectively (Lewis et al., $1982)$.

The other hearing organ in the frog is the basilar papilla (BP). This papilla is most sensitive to the highest frequencies within the hearing range of frogs (Feng

\footnotetext{
"We use the mone general tem "inner ear amplifer" since soveral watebrate classes do not possess a cochlea.
} 
et al., 1975). In Northern leopard frogs (Rana pipiens pipzens) it responds best to sounds of $1.2-2.4 \mathrm{kHz}$ (Ronken, 1991). The anatomy of the BP is unique among all classes of vertebrates (Wever, 1973); it contains between 50 and 100 hair cells that stand directly over the rigid cartilaginous wall of the papillar recess. Nearly all of these hair cells are covered by a tectorial membrane. In an individual animal, almost all BP nerve fibers exhibit very similar tuning curves, with characteristic frequencies (CFs) being essentially identical across nerve fibers (Ronken, 1990, Van Dijk et al., 1997). Also, afferent BP fibers are not suppressible (Feng et al., 1975), and the CF of BP fibers does not depend on temperature (Stiebler and Narins, 1990, Van Dijk et al, 1990). The unique anatomy and physiology of the BP suggest that tuning reljes on the mechanical properties of the structures in the papilla, without the contribution of electrical tuning from the heir cells (Smotherman and Narins, 1999b).

It has been hypothesized that of the two hearing papillae in the frog only the amphibian papilla (AP) incorporates an inner ear amplifier to enhance auditory functioning (Meenderink and Van Dijk, 2004). In contrast, auditory processing in the basilar papilla (BP) seemingly does not involve active amplification; this papilla is a passive hearing organ. This hypothesis was based on several observations that are linked to phenomena normally associated with the inner ear amplifier. [1] The generation of spontaneous otoacoustic emissions seems to be limited to the AP only (Van Dijk and Manley, 2001), and [2] DPOAEs evoked with low-level stimuli show different sensitivity to physiological insults, with high sensitivity for DPOAEs from the AP and less sensitivity for DPOAEs from the BP (Van Dijk et al, 2003). In conjunction with these observations, the growth of low-level DPOAE-amplitude with increasing stimulus levels is compressive (rate $\leq 1 \mathrm{~dB} / \mathrm{dB}$ ) in the AP while in the $B P$ it is expansive (rate $>1 \mathrm{~dB} / \mathrm{dB}$ : Meenderink and Van Dijk, 2004). If true, the passive nature of the $\mathrm{BP}$ places this hearing organ in a unique position within vertebrate hearing: no other vertebrate ear lacks an inner ear amplifier.

The aim of the present study was to test whether DPOAE generation in the $\mathrm{AP}$ and the BP involves an active inner ear amplifier, respectively. This was done by recording DPOAEs from both papillae over a range of body-temperatures. Since active processes are energy-dependent, the inner amplifier is energy-consuming. In a physiological setting, this energy must come from metabolism and hence will depend on temperature. Therefore, it is to be expected that the generation of DPOAEs is temperature-dependent. when an inner ear amplifier is involved in their generation. If the hypothesis is true, we expect to see a temperature-dependence of low-level DPOAEs from the AP, but no such dependence for DPOAEs from the BP. 


\subsection{Materials}

Distortion product otoacoustic cmissions (DPOAEs) were recorded from 9 ears of 5 Northern leopand frogs, Rana pipiens piphens; body weight: $42.6-62.0 \mathrm{~g}$ (mean: $52.5 \mathrm{~g}$ ). In order to immobilize the frog, it was anesthetized with an intramuscular injection of a pentobarbital sodium solution (diluted Nembutal; effective dosage $30 \mu \mathrm{g} / \mathrm{kg}$ body weight) in one of the hind limbs. DPOAEs were monitored using a custom-built probe that was carefully placed against the skin surrounding the tympanic membrane. A tight seal between the probe and the skin was obtained using vaselin. To monitor the frog's temperature, a small, K-type thermocouple, connected to a thermocouple reader (SR630, Stanford Research Systems), was placed inside the frog's orall cavity. Recordings were made inside a sound-attemating chamber, with the frog placed between wet gauze to prevent dehydration and to facilitate cutaneous respiration.

All procedures were approved by the animal experiment committee of the Maastricht University.

\subsubsection{DPOAE recording}

The sane experimental setup as described by Meenderink and Van Dijk (2004) was used to record DPOAEs. Briefy, the recording probe contained two miniature speakers (E-A-RTONE 3A, Aero Company) for stimulus delivery, and a 1 " high-sensitivity condenser microphone (type 4179 , Brüel \& Kjær) for emission recording. Emissions were evoked by simultaneously presenting two pure tones of slightly different frequencies $\left(f_{1}\right.$ and $f_{2}$, respectively) to the ear. Each of these tones was generated from a separate D/A channel (RP2, Tucker-Davis Technologies), attenuated (PA5, TDT) to set the desired stimulus level ( $L_{1}$ and $L_{2}$, respectively), and played from one of the speakers contained within the probe.

The microphone signal was passed through both a low-noise amplifier (type 2660, B\&K; amplification $=20 \mathrm{~dB}$ ) and a measuring amplifier (type 2610, B\&K; amplification $\geq 0 \mathrm{~dB}$ ) before being reconded on computer-disc using an $A / D$ channel (RP2, TDT). Stimulus tone generation and signał reconding were controlled by software written in MATLAB (The Mathworks) and RPVds (TDT). In order to monitor DPOAEs during the experiments, the spectrum of the amplified microphone signal was viewed online using a spectrum analyzer (SR760, SRS).

All DPOAE recordings were performed with $f_{2} / f_{1}=1.1$ and stimulus frequency $f$ chosen such that an integer number of sample points held exactly 100 periods of this tone. These periodic blocks of sample points also held an integer number of periods for the $f_{2}$ stimulus tone (110 periods), and all DPOAEs (e.g. 90 periods for $2 f_{1}-f_{2}$, and 120 periods for $2 f_{2}-f_{1}$ ). A series of these periodic blocks was recorded 
for each combination of stimulus tones. Artifact-free blocks (using a level-crossing artifact-rejection method) were averaged, and from this average, DPOAEs and noise were calculated using Fourier analysis.

In each ear investigated, the entire experiment consisted of the recording of several sets of DPOAEs, each set obtained at a different body-temperature. A single set of DPOAE-recordings entailed the mapping of the level-dependence of DPOAEs in both the amphibian papilla (AP) and the basilar papilla (BP). Hereto two $L_{1}, L_{2}$-maps were recorded, one from each papilla. Each $L_{1}, L_{2}$-map consisted of 81. recordings at different combinations of the two stimulus tones; $f_{1}$ and $f_{2}$ were fixed, while $L_{1}$ and $L_{2}$ were varied independently and randomly over a $32 \mathrm{~dB}$ range in $4 \mathrm{~dB}$ steps. For each ear, two combinations of stimulus frequencies were chosen to approximately match the frequencies that resulted in maximum DPOAE-amplitude in the AP and the BP, respectively. These were determined from a preliminary recorded DPOAE-audiogram and were not varied during the entire experiment. The stimulus-level ranges were $L_{1}=L_{2}=53 \ldots 85 \mathrm{~dB}$ SPL in the AP and $L_{1}=L_{2}=$ $56 \ldots 88 \mathrm{~dB}$ SPL in the BP.

\subsubsection{Experimental protocols}

Approximately 30 minutes after the administration of the anesthesia the frog was transferred to the sound-attenuating chamber. After placing the recording probe and the thermocouple a single set of DPOAE-recordings was obtained with the frog's body-temperature at room-temperature (approximately $21^{\circ} \mathrm{C}$ ). Subsequently, the animal was taken out of the set-up and placed in a small plastic container. This container was then floated for 10 minutes in a larger tank of cold water $\left(8^{\circ} \mathrm{C}\right)$, which cooled the frog to a temperature of approximately $12^{\circ} \mathrm{C}$ (the actual temperature decrease depended on the initial body-temperature and the body weight of the frog). After cooling, the frog was placed on a custom-built thermo-pad and the recording probe and thermocouple were reconnected. The temperature of the thermo-pad could be controlled actively by flowing water of known temperature (using a LAUDA RE106 thermobad) through a maze of tubes integrated in the pad, thus controlling the frog's body-temperature. Single sets of DPOAEs were recorded at different temperature settings (overall range: $12-23^{\circ} \mathrm{C}$ ), after allowing the body-temperature to stabilize for at least 5 minutes at each temperature setting.

To verify whether any of the observed effects were the result of the relatively rapid cooling of the frog, we also obtained sets of DPOAEs while slowly cooling the animal $\left(\leq 0.3{ }^{\circ} \mathrm{C} /\right.$ minute). In addition, one animal was rapidly cooled, after which its body-temperature was maintained at $12^{\circ} \mathrm{C}$ for 1 hour before reheating was started. The results from these experiments were similar to all other results, 
indicating that the observed effects were not greatly affected by the rate of bodytemperature change.

\subsection{Results}

The effects of body-temperature on DPOAE amplitude were similar in all ears studied. An example of these effects is illustrated in Fig. 4.1 for DPOAE at $2 f_{1}-f_{2}$ obtained from a single ear. The preliminary recorded DPOAE-audiogram (Fig. 4.1a) showed the typical bimodal dependence of emission amplitude on stimulus frequency that is associated with DPOAE generation in both the amphibian papilla (AP) and Whe basilar papilla (BP). Here, DPOAEs generated with $f_{1}<1250 \mathrm{~Hz}$ arose from the AP, while DPOAEs evoked with larger $f_{1}$ were from the BP. The two vertical dashed lines give the stimulus frequencies $f_{1}$ for which the shown $L_{1}, L_{2}$-maps were obtained. At room-temperature $\left(21^{\circ} \mathrm{C}\right.$; Figs. $\left.4.1 \mathrm{~b}, \mathrm{c}\right) L_{1}, L_{2}$-maps could be divided in two principal regions that showed a different stimulus-level dependence of DPOAE amplitude. This different level-dependence is illustrated by the different shape of the equal-amplitude contourlines. When at least one of the two stimulus levels was relam tively large, the consecutive contourlines were approximately straight lines, parallel to each other. In contrast, when both stimulus levels were relatively smail, contourlines were convex, encompassing a low-level DPOAE-ridge along which emission amplitude varied only marginally. Notice that this low-level ridge was much more pronounced in the AP than in the BP (compare Figs. 4.1b and c). We will refer to DPOAEs evoked with relatively small stimulus levels as low-level DPOAEs and to DPOAEs evoked with one or both stimulus levels relatively large as high-level DPOAES.

When body-temperature was decreased $\left(12^{\circ} \mathrm{C}\right)$, the $L_{1}, L_{2}$-map obtained from

Figure 4.1: (opposite page) Overview of the temperature dependence of $2 f_{1}-f_{2}$ DPOAE amplitudes from a single ear. Panel (a) shows the DPOAE-andiogram initially recorded at room-temperature $\left(T=21^{\circ} \mathrm{C}\right)$. The stimulus parameters were $L_{1}=L_{2}=76$ $\mathrm{dB} S \mathrm{SP}$ and $f_{2} / f_{1}=1.1$. The bimodal shape of the DPOAE-audiogram reflects emission generation from the amphibian papilla $\left(\mathrm{AP} ; f_{1}<1250 \mathbb{H} z\right)$ and the basitar papilla (BP; $f(>1250 \mathrm{~Hz})$, respectively. The gray area indicates the noise floor. The remaining panels contain $L_{1}, L_{2}-$ maps from the amphibian papilla $\left(f_{1}=911 \mathrm{~Hz}\right.$; left dashed line in panel a) and the basilar papilla ( $f_{1}=1719 \mathrm{~Hz}$; right dashed line in panel a). These $L_{1}, L_{2}$-maps were obtained at three subsequent body-temperatures: $T=21{ }^{0} \mathrm{C}, 12{ }^{\circ} \mathrm{C}$, and back to $21{ }^{\circ} \mathrm{C}$, respectively. In each map, contourlines are drawn at $5 \mathrm{~dB}$ increments, with the appropriate levels given in the krey. The emboldened contourlines in panels (b) and (c) are also displayed in Figs. $4.2 \mathrm{a}, \mathrm{b}$, respectively. 


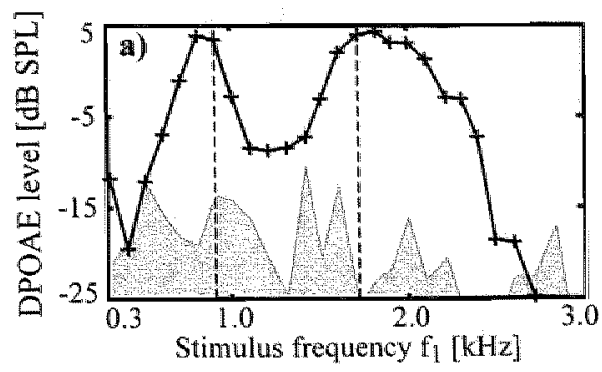

Amphibian papilla; $\mathbb{f}_{\mathbb{1}}=911 \mathrm{~Hz}$

Basilar papilla; $f_{1}=1719 \mathrm{~Hz}$
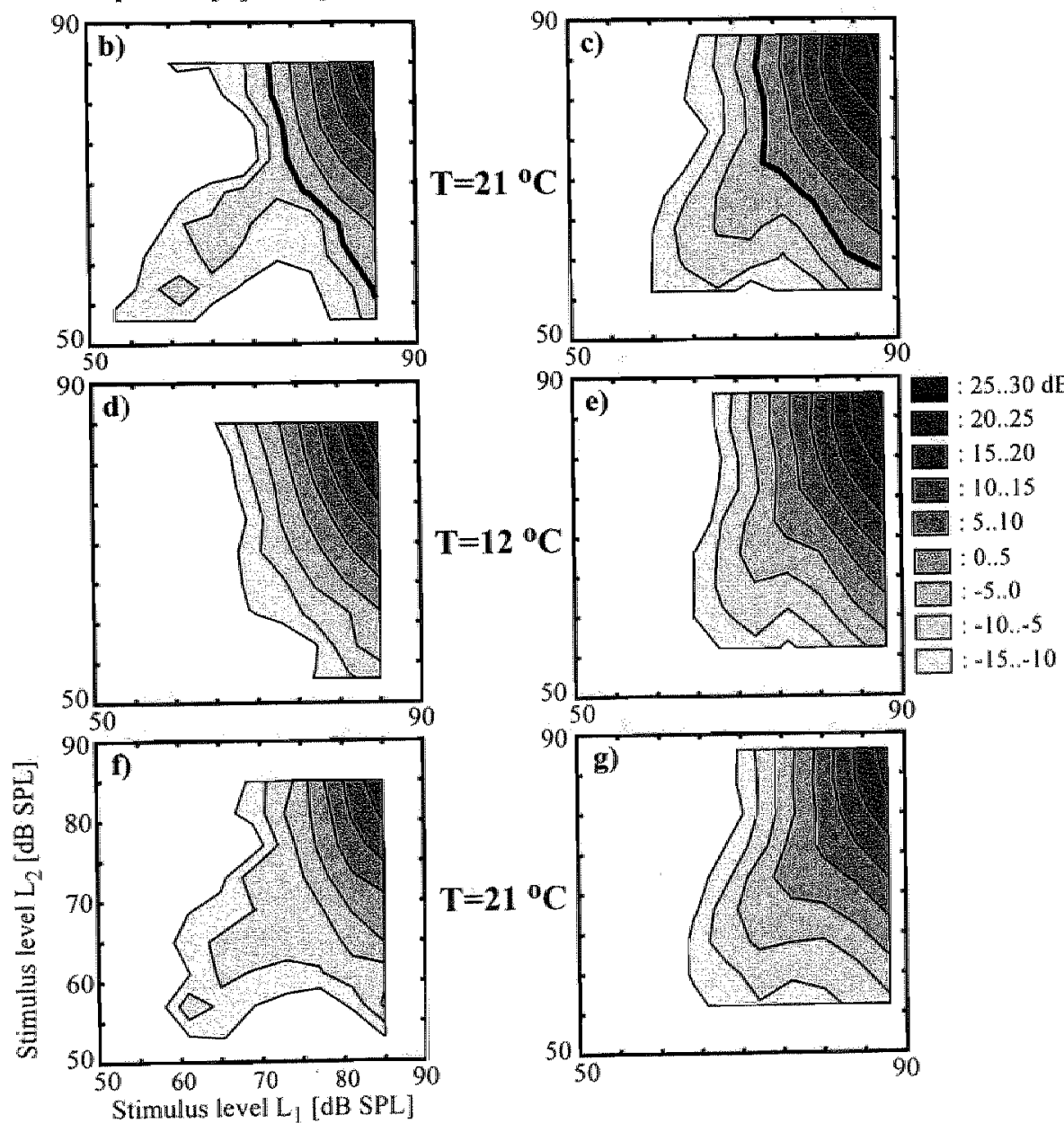

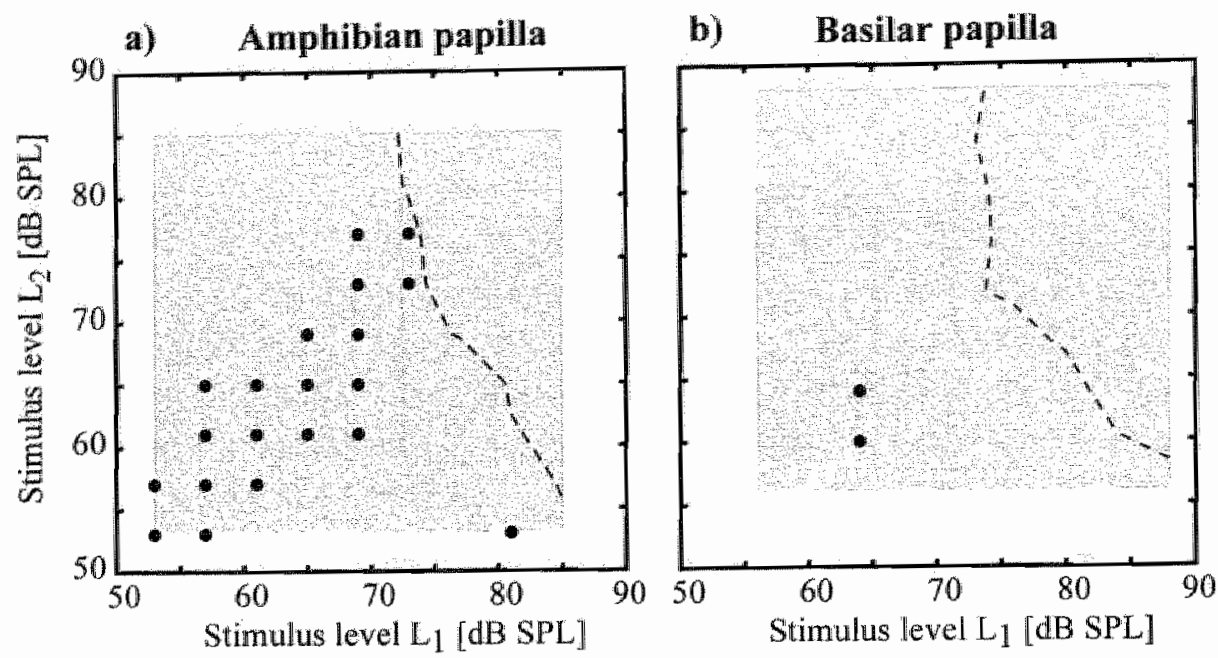

Figure 4.2: Pair-wise comparison (Wilcoxon signed-rank test) between DPOAEamplitudes from the temperature categores $11.5^{\circ} \mathrm{C} \leq T<14.5^{\circ} \mathrm{C}$ and $20.5^{\circ} \mathrm{C} \leq T^{\circ}<23.5$ ${ }^{a} \mathrm{C}$ in (a) the amphibian papilla and (b) the basilar papilla. $2 f_{1}-f_{2}$ and $2 f_{2}-f_{1}$ DPOAEs were combined in the analysis. The filled circles (' $)$ denote $\left(L_{1}, L_{2}\right)$-combinations for which the DPOAE anplitude was significantly different $(p<0.05)$ between the two temperature categories. For reference, the shaded areas represent the $L_{1}, L_{2}$-areas for which DPOAEs were obtained. The dashed lines in panels (a) and (b) are the emboldened con tourlines from Fig. $4.1 \mathrm{~b}$,c, respectively, and approximately give the separation between the high-level and the low-level region in the $L_{1}, L_{2}$-maps.

the AP (Fig. 4.1d) was markedly different from the one obtained at room-temperature. At this body-temperature, the convex, low-level contourlines were absent: the reduced levels of the low-level emissions no longer exceeded the estimated noise floor. In contrast, the straight, high-level contourlines were still present, with emission levels comparable to the high-level DPOAE amplitudes obtained at room-temperature. In the BP, the decrease in body-temperature did not result in any clear changes of DPOAE amplitude for any stimulus level combination represented in the $L_{1}, L_{2}-$ map (Fig. 4.1e). Here, both the convex, low-level, as well as the straight, high-level contounlines were present. Also, the absolute DPOAE levels were approximately identical to the levels found at room-temperature within the entire $L_{1}, L_{2}$-map.

Finally, when body-temperature was returned to room-temperature $\left(21^{0} \mathrm{C}\right)$, the obtained $L_{1}, L_{2}$-maps were similar to those obtained before cooling. In both the AP and the BP a low-level and a high-level region could be identified based on the shape of the contourlines. Also, the levels of the DPOAEs in the entire $L_{1}, L_{2}$-maps were comparable to the mplitudes obtained before cooling.

In order to compare the results obtained from different ears, $L_{1}, L_{2}$-maps were 
grouped in four categories based on body-temperature: [1] $11.5{ }^{\circ} \mathrm{C} \leq T^{4}<14.5$ ${ }^{a} \mathrm{C}_{3}[2] 14.5{ }^{\circ} \mathrm{C} \leq T<17.5{ }^{\circ} \mathrm{C} ;[3] 17.5^{\circ} \mathrm{C} \leq T<20.5{ }^{\circ} \mathrm{C} ;$ and $[4] 20.5{ }^{\circ} \mathrm{C} \leq$ $T^{\prime}<23.5^{\circ} \mathrm{C}$. In Fig. 4.2 the DPOAE amplitudes from the two extreme temperature categories (i.e. $11.5^{\circ} \mathrm{C} \leq T<14.5{ }^{\circ} \mathrm{C}$ versus $20.5^{0} \mathrm{C} \leq T<23.5{ }^{\circ} \mathrm{C}$ ) at all $L_{1}, L_{2}$ combinations are compared. Both DPOAEs at $2 f_{1}-f_{2}$ and $2 f_{2}-f_{1}$ were included in the comparison. Significant differences (Wilcoxon signed-rank test; $p<0.05$ ) between the amplitudes were only found for emissions along the low-llevel ridge in the AP (Fig. 4.2a). Again, high-level DPOAEs from the AP did not significantly vary in amplitude with change in body-temperature. In the $\mathbb{B P}$, two low-level $I_{1}, L_{2}$ combinations did show significant differences in DPOAE amplitude between the two temperature categories.

The relationship between body-temperature and relative $2 f_{1}-f_{2}$ DPOAE amplitude is further illustrated in Fig. 4.3 for four different $L_{1}, L_{2}$ combinations. We considered the relative amplitude (i.e. amplitude minus the mean DPOAE amplitude at the fixed $L_{1}, L_{2}$ combination averaged across temperatures) as function of body-temperature, since the absolute DPOAE amplitudes showed great variability from one ear to another. The same four temperature-categories from above were used to combine the $L_{1}, L_{2}$-maps from different ears. Only DPOAEs exceeding the noise floor ( $-17 \mathrm{~dB}$ SPL) were included in the calculations. The only significant correlation between emission amplitude and body-termperature was found for low-level DPOAEs from the AP (Eig. $4.3 \mathrm{c} ; r=0.58 ; p<0.05$ ). Here, relative DPOAE amplitude decreased with decreasing body-temperature. For high-level DPOAEs from the AP (Fig. 4.3a), as well as for low- and high-level DPOAEs from the BP (Figs. 4.3b and $d$, respectively) changes in relative emission amplitude were not significantly related to body-temperature.

The results presented in Fig. 4.3 include only data points that exceded the estimated noise floor. The data points that decreased below detectable levels with changing body-temperature were thus ignored. But these changes also signify a temperature-dependent decrease in DPOAE amplitude. In Fig. 4.3, the mubers in parentheses give the number of ears that emitted detectable emissions at each temperature category. At room-temperature $\left(22^{\circ} \mathrm{C}\right)$, all nine ears emilted low-and high level DPOAEs from both the AP and the BP. With decreasing body-temperature, the number of ears that emitted low-level DPOAEs from the AP decreased. Such a decrease in the number of emitting ears was absent for high-level DPOAEs in both the AP and the BP. For low-level DPOAFs from the BP, only one ear did not emit low-level DPOAEs at $13{ }^{\circ} \mathrm{C}$. This ear emitted just above the noise $100 \mathrm{r}$ at: room-temperature.

In summary, decreases in body-temperature only affected the generation of lowlevel DPOAEs from the AP by reducing their amplitudes, often resulting in a loss 
Amphibian papilla

a) $\mathrm{L}_{1}=\mathrm{L}_{2}=81 \mathrm{~dB} \mathrm{SPL}$

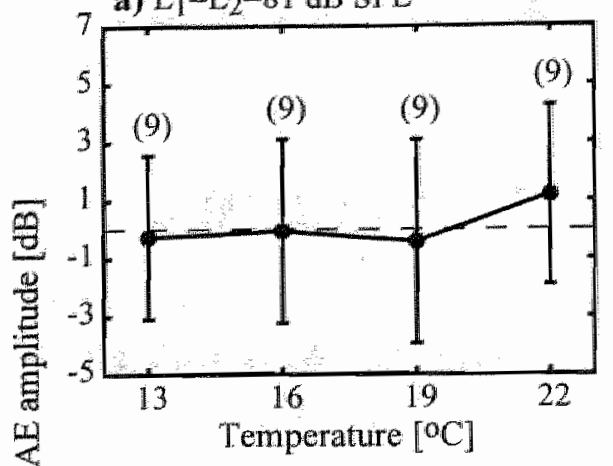

c) $\mathrm{L}_{1}=\mathrm{L}_{2}=69 \mathrm{~dB} \mathrm{SPL}$

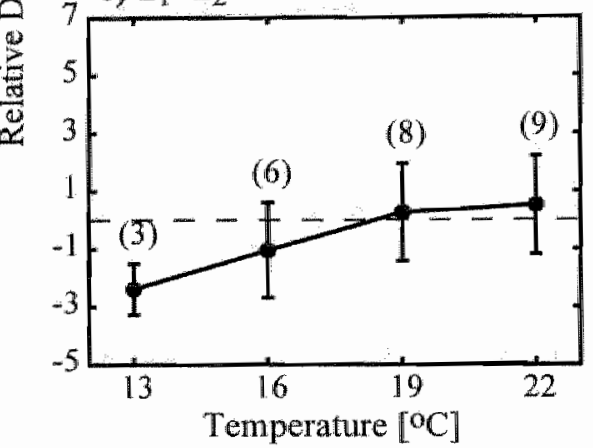

Basilar papilla

b) $\mathrm{L}_{1}=\mathrm{L}_{2}=80 \mathrm{~dB} \mathrm{SPL}$

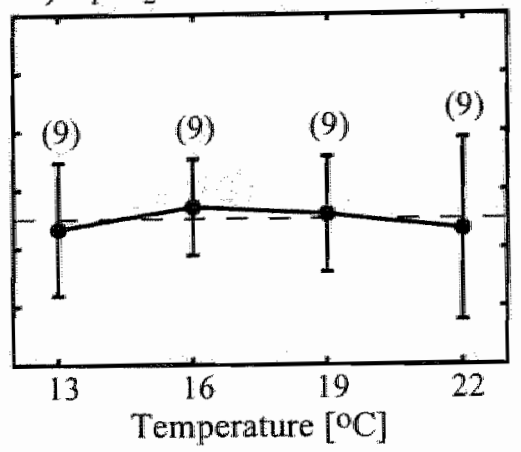

d) $L_{1}=72 \& L_{2}=68 \mathrm{~dB}$ SPL

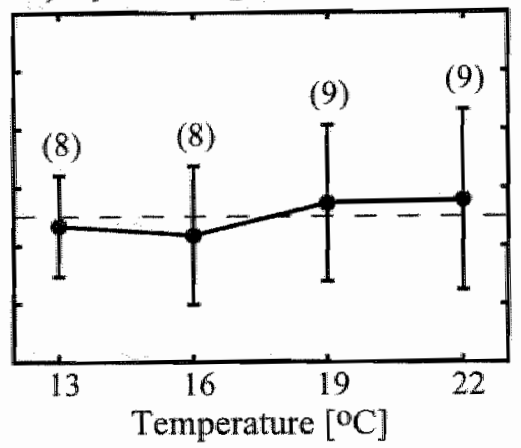

Figure 4.3: Relative amplitude for DPOAEs at $2 f_{1}-f_{2}$ versus body-temperature. For each ear, DPOAE amplitude is relative to the average DPOAE amplitude at the fixed $L_{1}, L_{2}$ combination, averaged across temperatures. These were used since absolute DPOAE levels varied greatly from one ear to another. Data points were grouped in four temperature categories and the mean \pm S.D. for each category is shown. The left collumn gives results for the amphibian papilla, while the right column giwes results for the basilar papilla. al) High-level DPOAES from the AP: $L_{1}=L_{2}=81 \mathrm{~dB}$ SPL. b) High-level DPOAEs from the BP: $L_{1}=L_{2}=80 \mathrm{~dB}$ SPL. c) Low wevel DPOAEs from the AP: $L_{1}=L_{2}=69 \mathrm{~dB}$ SPL. d) Low level DPOAJs from the BP: $L_{1}=72$ and $L_{2}=68 \mathrm{~dB}$ SPL. The only significant correlation between relative DPOAE amplitude and body-temperature was found for the Jownlevel DPOAEs from the AP $(r=0.58 ; p<0.05)$. In each subpanel, only data points that exceded the estimated noise floor were included. The numbers in parentheses give the number of ears that net this criterion.

of these emissions. This level-reduction was reversed when body-temperature was returned to room-temperature. For both high-level DPOAEs from the AP as well as for all DPOAEs from the BP no significant changes in the DPOAE amplitudes were observed with changing body-temperature. Their absolute levels did not change, 
nor did the shape or orientation of the contourlines in $L_{1}, L_{2}-$ maps.

\subsection{Discussion}

These results have important implications concerning the presence of an imner ear amplifier within the anuran ear. The inner ear amplifier is an active mechanism, which relies on metabolic energy for its proper functioning. Consequently, its functioning must depend on temperature. Moreower, the amplitude of DPOAEs which are generated or boosted by an active amplifier must also depend on body-temperature. The insensitivity of DPOAE amplitude from the basilar papilla to temperaturechanges implies that the generation of these emissions cannot involve an active inner ear amplifier. In contrast, the temperature-dependence of low-level DPOAEs from the amphibian papilla is consistent with the presence of an active amplification mechanism in this hearing organ.

These conclusions are consistent with other observations on the difference between the amphibian and the basilar papilla: [1] the generation of spontaneous otoaconstic emissions (SOAEs) is limited to the AP only (Van Dijk and Manley, 2001), and [2] low-level DPOAEs from the AP show a higher vulnerability to physiological insults than low-level DPOAEs from the BP (Van Dijk et al, 2003). These differences suggest an important difference in the functioning of the two hearing papillae.

An additional difference between the $A P$ and the $B P$ is the growth rate of lowlevel DPOAE amplitude with increasing stimulus levels. In the BP, DPOAE amplitude grows expansive with stimulus levels. In contrast, in the AP and in the hearing organs of most other tetrapods (reviewed in Meenderink and Van Dijk, 2004), DPOAE amplitude grows compressive with a slope of about $1 \mathrm{~dB} / \mathrm{dB}$. Maybe, the compressive DPOAE-growth is a universal property of the inner ear amplifer.

In addition to the temperature effects on the inner ear amplifier, other mech anisms may also be affected by the changed body-temperature, thus changing the emission-levels. For instance, decreasing the body-temperature in rats reduces the compliance of the middle ear (Geal-Dor et al., 1997), resulting in a small conductive loss with an air-bone gap of $6 \mathrm{~dB}$ (Khvoles et al, 1998). This conductive loss will reduce both the amplitudes of the two stimulus tones upon entering the inner ear, as well as the emission levels that are transmitted to the extermat ear. But such a loss will equally affect DPOAE levels evoked with any combination of stimulus levels, resulting in an overall decrease of DPOAE levels in the $L_{1}, L_{2}$-maps. Our results showed this not to be the case in frogs; the amplitudes of high-level DPOAEs from the AP, as well as the amplitude of all DPOAEs from the BP were not depressed when temperature decreased (Fig. 4.3). In fact, the exclusive sensitivity of low level 
DPOAE from the AP indicates that temperature-indnced overall changes in the inner or middle ear cannot account for this sensitivity, since these changes would also affect high-level DPOAEs from the AP. Rather, it seems that the temperature directly influences the functioning of the inner ear amplifer, whose functioning is most arital for low-level stimuli.

Several studies have explored the ffects of body-temperature on transient-evoked OAEs and/or DPOAEs in mammals (Ferber-Viant et al, 1995; Noyes et al., 1996; Veuillet et al, 1997; Knvoles et al. 1998). Results from these studies indicate that fi changes in body-temperature affect DPOAEs, this primarily occurs for emissions evoked with moderate-to-low stimulus intensities and for temperatures sufficiently altered from normal. In effect, it seems that there exists a range of temperatures wherein OAEs are not correlated with temperature. Outside this temperaturerange, both for lypo- and hyperthermia, the amplitudes of low-level emissions are depressed. It is not clear from our results whether there exists a range of bodytemperatures wherein low-level DPOAEs from the AP are uncorrelated with bodytemperature. Although there is a significant correlation between these emission amplitudes and body-temperature, Fig. $4.3 \mathrm{c}$ may indicate that there is no amplitudedifference between DPOAEs evoked at $19^{\circ} \mathrm{C}$ and $22^{\circ} \mathrm{C}$. For SOAEs from the anuran ear it has been suggested that temperature functions as a sort of "on-ofl" switch for emission generation (Van Dijk et al., 1989); below a certain temperature emissions were "switched of" "while for higher temperatures emissions were "switched on" but their amplitudes did not depend on temperature. This suggests that there may exist a temperature-range in the frog wherein emission amplitudes are uncorrelated with temperature, similar as found in mammals.

Does the lack of temperature-dependence of DPOAEs from the BP imply that this auditory organ functions fully passively? "The answer is "no". An active inputoutput system is defined by the fact that the output-signal contains energy that: does not derive directly from the input-signal. In hair cells, the input is the deflection of the hair bundles, and the output is a transduction current through the ion-channels in the hair cell's membrane. This current is gated by the imput-signal, but its energy is derived from an ion concentration-gradient across the cell's membrane. Consequently, the forward mechano-electrical transduction in hair cells is an active mechanism. The insensitivity of DPOAEs from the BP to temperaturechanges implies that apparently the forward transduction is not directly involved in thein generation. Similar to forward transduction, reverse (electro-mechanical) transduction in hair cells is also active. The temperature-insensitivity of DPOAEs from the BP indicates that reverse transduction does not take place in this hearing organ. Consequently, DPOAEs in the BP must result from nonlinear reflectances, which may involve the nonlinear stiffness of hair bundles (Howard and Hudspeth, 
1988).

Our results place the basilar papilla of anurans in a unique postion among vertebrates; distinct from the amphibian papilla and from the inner ears of wll other vertebrates, the basilar papilla may be the only hearing organ without active feedback.

The question arises how the frog may benefit from a passive ear. Seemingly, the absence of active amplification would reduce both the sensitivity and frequencyselectivity of the papilla. However, nerve fibers innervating the BP both have characteristic levels (CL: the lowest stimulus level that still evokes a response in the nerve) and tuning sharpness ( $Q_{10}$ : the scaled frequency-width of the tumingcurve at $10 \mathrm{~dB}$ above $\mathrm{CL}$ ) similar to those found for nerve fibers from the $\mathrm{AP}$ (Ronken, 1991). Apparently, the conjoint functioning of hair cells within the BP make this entire hearing organ "as good as" individual tonotopic locations within the AP. In addition, the absence of an inner ear amplifier may even be beneficial to the frog. Given the poikilothermic nature of amphibians, their body-temperatures match the ambient temperature, which may fluctuate considerably. The absence of a temperature-dependent inner ear amplifier makes that the functioning of the BP is not hindered by these temperature-fluctuations: the spectral window of auditory input is consistently transduced in the basilar papilla, independent of ambient and/or body-temperature.

\subsection{Acknowledgements}

This work was supported by grants from the Netherlands Organization for Scientific Research (NWO), and the Heinsius Houbolt Foundation. 
Chapter 5

\section{Comparison between distortion}

product otoacoustic emissions and

nerve fiber responses from the

basilar papilla of the frog

\section{Abstract}

The basilar papilla (BP) is one of the three end organs in the frog inner ear that is sensitive to airborne sound. Its anatomy and physiology are unique among all classes of vertebrates. Essentially, the BP functions as a single auditory filter presumably arising from a mechanically-tuned mechanism. Ass such, both neural and distortion product otoacoustic emission (DPOAE) tuning may reflect a single mechanical filtering mechanism. Using the Duffing cscillator as a simple model for both neural and DPOAE tuning from the BP, two predictions can be made: [1] the characteristic frequency (CF) of neural tuning and the best frequency (BF) of DPOAE tuning will coincide and [2] the neural tuning curve and DPOAE-andiogram have a similar shape when the neural tuning curve is scaled by a factor of 4 along the $y$-axis. We recorded both neural tuming curves and DPOAE-andiograms from the BP of the leopard frog. These recordings show good agreement with the model predictions when the stimulus tones are related by relatively small stimulus frequency ratios. For larger stimulus frequency ratios, DPOAE recordings clearly deviate from model predictions. These differences are most likely caused by the oversimplified representation of the frog $\mathrm{BP}$ by the model. 


\subsection{Introduction}

The andomy of the inner ear exhibits a high degree of variability across the various classes of vertebrates. Notwithstanding this, the transformation of sound pressure into activity in the auditory nerve ultimately involves the same specialized cells in all vertebrates: hair cells.

In frogs, hair cells that are sensitive to airbome sound are distributed over three anatomically-separated papillae, each one most sensitive to a different range of frequencies. The secculus (S) is sensitive to high-level sounds at very low frequencies. No tonotopic organization has been reported for the frog sacculus. The amplibian pepilla (AP), which is most sensitive to the low- and mid-frequencies within the frog hearing range, consists of an elongated strip of hair cells. These cells are directly over the rigid wall of the papillar recess, rather than over a flexible membrane (as in the cochlea). Overlying the AP hair cells is a tectorial membrane. Both the shape of this membrane as well as its position within the papillar recess suggest that it plays an important role in the transcluction of sound by hair cells (Lewis and Leverenz, $1983)$. The papilla exhibits tonotopic organization with the highest $(\approx 1.2 \mathrm{kHz})$ and lowest $(\approx 0.1 \mathrm{kHz}$ ) frequencies being represented caudally and rostrally, respectively (Lewis et al., 1982).

The third hearing organ in the frog is the basilar papilla (BP). This papilla is most sensitive to the highest frequencies within the hearing range of frogs (Feng et al. 1975). In leopard frogs (Rana pipiens pipiens) it responds best to sounds of $1.2-2.4 \mathrm{kHz}$ (Ronken, 1991). The anatomy of the BP is unique among all classes of vertebrates (Wever, 1973); it consists of approximately $50-100$ hair cells standing directly over the rigid cartilaginous wall of the papillar recess. Almost all of these hair cels are covered by a tectorial nembrane. In an individual animal, amost all $B P$ nerve fibers exhibit very similar tuning curves, with characteristio frequencies (CFs) being assentially identical across nerve fibers (Ronken, 1990, Van Dijk et al., 1997). Also, afferent $\mathrm{BP}$ fibers are not suppressible (Feng et al, 1975), and the CF of BP fibers does not depend on temperature (Stiebler and Narins, 1990, Van Dijk et al., 1990). Although it is not completely understood how tuning is achieved in this organ, the unique anatomy and physiology of the BP suggest that tuning relies on the mechanical properties of the structures in the papilla, without the contribution of electrical tuming from the hair cells (Smotherman and Narins, 1999b).

Upon stimulation with two tones that have appropriately chosen frequencies $\left(f_{1}, f_{2}\right)$ and levels $\left(L_{1}, L_{2}\right)$ the basilar papilla produces distortion product otoacoustic emissions (DPOAEs; Van Dijk and Manley, 2001). It has been hypothesized that these DPOAEs are the result of the passive nonlinear properties of the anatomical structures (i.e. hair cells, the tectorial membrane) found within this organ (Meen- 
derink and Van Dijk, 2004). So, similar to the nerve, tuming of DPOAEs seems to be the result of a mechanically-tumed filter. Thus, both neural and DPOAE tming may reflect the same, single auditory filter, with the tuning having a mechanical origin.

Recently, the Dufing oscillator has been used as a simple model bor DPOAEs from the BP (Van Dijk and Manley, 2001, Meenderink et al., 2005). These studies show that such a simple model qualitatively describes the dependence of emission amplitude and phase on both absolute $\left(f_{1}, f_{2}\right)$ and relative $\left(f_{2} / f_{1}\right)$ stimulus frequencies. In the present study we used the Duffing oscillator as a model for both DPOAEs and neural tuning from the $\mathrm{BP}$. We show that [1] the $\mathrm{CF}$ of the neural tuning curve and the stimulus frequency resulting in maximum distortion product amplitude coincide. This correlation is independent of the ratio $f_{2} / f_{1}$ used to evoke DPOAEs; and [2] the shapes of the neural tuning curves and the DPOAE-audiograms are markedly different: the neural tuning curves are much broader than the DPOAE-audiograms. However, when the neural tuning curves are scaled by a factor of 4 along the $y$-axis, the two curves become almost identical in shape.

We tested these two model predictions by obtaining both neural tuning curves and several DPOAE-audiograms from the basilar papilla of the leopard frog $R$. pipiens pipiens. When using relatively small stimulus frequency ratios $\left(f_{2} / f_{1}<1.16\right)$ during DPOAE recordings, the results closely agree with the model predictions. For larger ratios this is not the case. This difference is most likely caused by the oversimplified representation of the frog $\mathrm{BP}$ in the used model.

\subsection{Methods}

\subsubsection{Animal care}

Recordings were made from the left ear of the Northern leopard frog Rana pipuens pipiens: $\mathrm{n}=9$; body mass: $17.7-31.9 \mathrm{~g}$ (mean $24.7 \mathrm{~g}$ ); snout-vent length: $58.6-78.4$ mm (mean: $67.9 \mathrm{~nm}$ ). Due to the duration of the experiments, nerve fiber recordings were performed the day following the DPOAE recordings. Prior to both DPOAE and nerve fiber recordings the animal was anesthetized by intramuscular mjection of sodium pentobarbital (Nembutal: $60 \mathrm{mg} / \mathrm{ml} ; 1.0 \mu \mathrm{l} / \mathrm{g}$ body mass) in one of the hind limbs. If necessary, supplementary anesthesia were administered during the experiments. Recordings were performed inside a sound-attenuating room, with the frog placed on a vibration isolation table. To prevent dehydration and to facilitate cutaneous respiration during the experiments, the animal was covered by gauze soaked in frog Ringer's solution. At the end of the nerve-fiber experiment, animals were killed by restruction of the central nervous system (double-pith procedure). 
The "Principles of Care" (NHH publication 85-32, revised 1985) and USA regulations were followed throughout this study, and protocols were approved by the University of California Animal Research Committee.

\subsubsection{DPOAE recordings}

Distortion product otoacoustic emissions (DPOAEs) were recorded using a setup and method described previousiy (Meenderink et al., 2005). Briefly, after anesthetizing the animal, the open end of a custom-built probe was sealed to the skin surrounding the tympanic membrane using silicone grease. The probe contained two miniature speakers (ER-10C, Etymotic Research) for stimulus delivery and one $\frac{1}{2}$ " condenser microphone (Brüel \& Kjar type 4134), connected to a pre-amplifier (B\&K type 2069; $60 \mathrm{~dB}$ amplification), for emission recording. In order to evoke DPOAEs, two stimulus tones with frequencies $f_{1}$ and $f_{2}$ (where $f_{1}<f_{2}$ ) and levels $L_{1}$ and $L_{2}$ were played simultaneously, one from each speaker. All DPOAE recordings were made with stimulus levels $L_{1}=L_{2}=76 \mathrm{~dB}$ SPL. The stimulus frequency $f_{1}$ was systematically increased from 213 to $2774 \mathrm{~Hz}$ in approximately $30 \mathrm{~Hz}$ steps. This $f_{1}$ sweep was repeated 11 times, each sweep with the frequency ratio $f_{2} / f_{1}$ fixed at a different value $\left(f_{2} / f_{1}\right.$ varied from 1.02 to 1.4 , respectively).

The generation of the stimulus tones, as well as the recording of the microphone signal was controlled by at desktop computer, using software written in MATLAB (The Mathworks, Inc.) and RPvds (Tucker Davis Technologies). This software controlled two separate D/A channels (RP2, TDT) and two programmable attenuators (PA5, TDT) for stimulus generation, and an A/D channel (RP2, TDT) used for recording of the microphone signal.

The two stimulus frequencies were chosen such that they were periodic over the same integer number of sample points. This ensures that the DPOAE frequencies are also periodic over that same number of sample points, resulting in sample blocks in which all frequencies of interest $\left(f_{1}, f_{2}\right.$ and $\left.f_{\text {dpose }}\right)$ are periodic. For each stimulus frequency pair, $f_{1}, f_{2}$, a series of these blocks was recorded. After artifact rejection and averaging of these blocks the levels of the stimulus tones and the DPOAEs were calculated by Fourier analysis.

\subsubsection{Nerve fiber recordings}

The day after the DPOAE recordings, the frog was re-anesthetized (using the same dosage of anesthesia as during the DPOAE recordings) in preparation for the recordings from the VIIIth nerve. Before the animal was placed inside the sound-attenuating room the auditory nerve that innervates the left ear was surgically exposed using a ventral approach (Capranica and Moffat, 1975, Zelick and Narins, 1985). The 
frog was placed on its back and the lower jaw was retracted to expose the roof of the mouth. After covering the frog with wet gauze a small hole was drilled on the lateral side of the braincase, thus exposing the VIIIth nerve and its point of entry into the brain. After surgery, the animal was transferred to the sound-attenuating room to conduct the experiments. For the intracellular recordings from individual nerve fibers we used conventional glass electrodes filled with $3 \mathrm{M} \mathrm{KOL} \mathrm{(resulting} \mathrm{in}$ an impedance typically $>40 \mathrm{M} \Omega$ ). Under visual control, the tip of the electrode was placed over the surface of the auditory nerve and advanced into the nerve using a remotely-driven hydraulic microdrive (Narishige MO-103L). The signal from the electrode was fed through a high input impedance pre-amplifier (Winston Electronics, model 1000) and a spike analyser (Mentor N-750) before being digitized using an A/D channel (DD1, TDT).

Pure-tone stimuli $(0.3-5.0 \mathrm{kHz})$ were generated from a D/A channel (DD1, TDT), set to the correct level using a programmable attenuator (PA4, TDT) and presentied to the operated ear through a dynamic speaker (Beyer DT48). This speaker was coupled to the frog ear via a brass housing and a short piece of rubber tubing. A tight seal between the tubing and the skin surrounding the tympanic membrane was obtained using silicone grease. The brass housing also contained a $\frac{1}{2}$ " condenser microphone (Brüel \& Kjær type 4134), which was used to calibrate the level of the stimulus tone.

While advancing the electrode through the nerve using the microdrive, the ear was stimulated using short bursts of white rioise. As soon as a nerve fiber was encountered an automated staircase algorithm was used to obtain two frequency threshold curves (FTCs) using an "up-down" threshold-tracking method (Evans, 1979): once from low to high frequencies, and once from high to low frequencies. The automated system consisted of software running on a desktop computer and controlled both stimulus generation and data acquisition. The two FTCs were recorded with a frequency resolution of 40 points/octave and a $1 \mathrm{~dB}$ intensity resolution. Their average was calculated and subsequently smoothed using a moving Hanning window (window width $=1 / 8$ octave).

\subsection{Theory: the Duffing oscillator}

As a simple model for the basilar papilla, we will consider the Duffing oscillator. This oscillator has been used previously to model distortion product otoacoustic emissions from the BP (Van Dijk and Manley, 2001, Meenderink et al., 2005). Although the Duffing oscillator is an (over)simplification of the basilar papilla mechanics, it models remarkably well the relation between the amplitude and phase of the DPOAE, and the stimulus frequencies $f_{1}$ and $f_{2}$ (Meenderink et al, 2005). The Duffing oscillator 
is described by the second-order nonlinear equation

$$
m \ddot{x}+R \dot{x}+k(x) x=F(t),
$$

where $m$ is a mass, whose movement is driven by the force $F(t)$. This movement is impeded by both a resistance $R$, and a nonlinear stiffness $k(x)=k_{o}\left(1+\frac{x^{2}}{x_{\sigma}^{2}}\right)$.

In order to evoke distortion products, the oscillator is driven by a two-tone force

$$
F(t)=A_{1} \sin \left(2 \pi f_{1} t\right)+A_{2} \sin \left(2 \pi f_{2} t\right),
$$

where $A_{1}$ and $A_{2}$ are the amplitudes of the two tones at frequencies $f_{1}$ and $f_{2}$ $\left(f_{2}>f_{1}\right)$, respectively. We calculated distortion products for a range of two-tone combinations: $f_{1}$ varied between 0.2 and 2.0 in 0.01 steps, with the normalized resonant frequency being 1 , while $f_{2} / f_{1}$ was between 1.02 and 1.4 in steps of 0.01 . Figs. 5.1a,b show DP amplitude when $n=1, r=1, k_{o}=4 * \pi^{2}, x_{o}=1$ and $A_{1}=A_{2}=1$. Here, individual lines give DP amplitude, obtained with a fixed$f_{2} / f_{1}$, as function of stimulus frequency (either $f_{1}$ for $2 f_{1}-f_{2}$, or $f_{2}$ for $2 f_{2}-f_{1}$ ). It can be seen that for $2 f_{1}-f_{2}$ (Fig. 5.1 a), each DP-audiogram has its maximum when $f_{1}$ coincides with the resonant frequency of the osciliator, as indicated by the filled circles. In contrast, for $2 f_{2}-f_{1}$ (Fig. 5.1b) maxima in the different DP-audiogramsi occur when $f_{2}$ coincides with the oscillator's resonant frequency (filled circles).

The Duffing oscillator was also used to model frequency threshold curves (FTC) in the basilar papilla. An FTC is a function that specifies for each frequency $f$ the corresponding amplitude $A$ required to reach a threshold response in the nerve fiber. Typically, the threshold is defined as an increase in the number of spikes above the spontaneous firing that occurs during a short interval of time. Rather than adding some spike-generator to the model, we will assume that the FTC is inversely proportional to the frequency-response curve of the Duffing oscillator, as expressed by the movement $x$ (Eq. 5.1). This will be approximately true for relatively low stimulus levels, when the growth of the response (i.e. spike rate) with increasing stimulus level is (approximately) linear. The inverted frequency-response function for the Duffing oscillator is shown in Fig. 5.1c (black dashed line) for the same parameters used for the generation of distortion products. The response curve was computed by driving the oscillator by a fixed-amplitude, one-tone force

$$
F(t)=A \sin (2 \pi f t),
$$

where $A$ and $f$ are the amplitude and the frequency of the stimulus, respectively. The characteristic frequency $(C F$, i.e. the stimulus frequency that requires the lowest stimulus level $A$ for which movement $x$ reaches threshold) of the FTC coincides with the resonant frequency $(f=1)$ of the oscillator. 

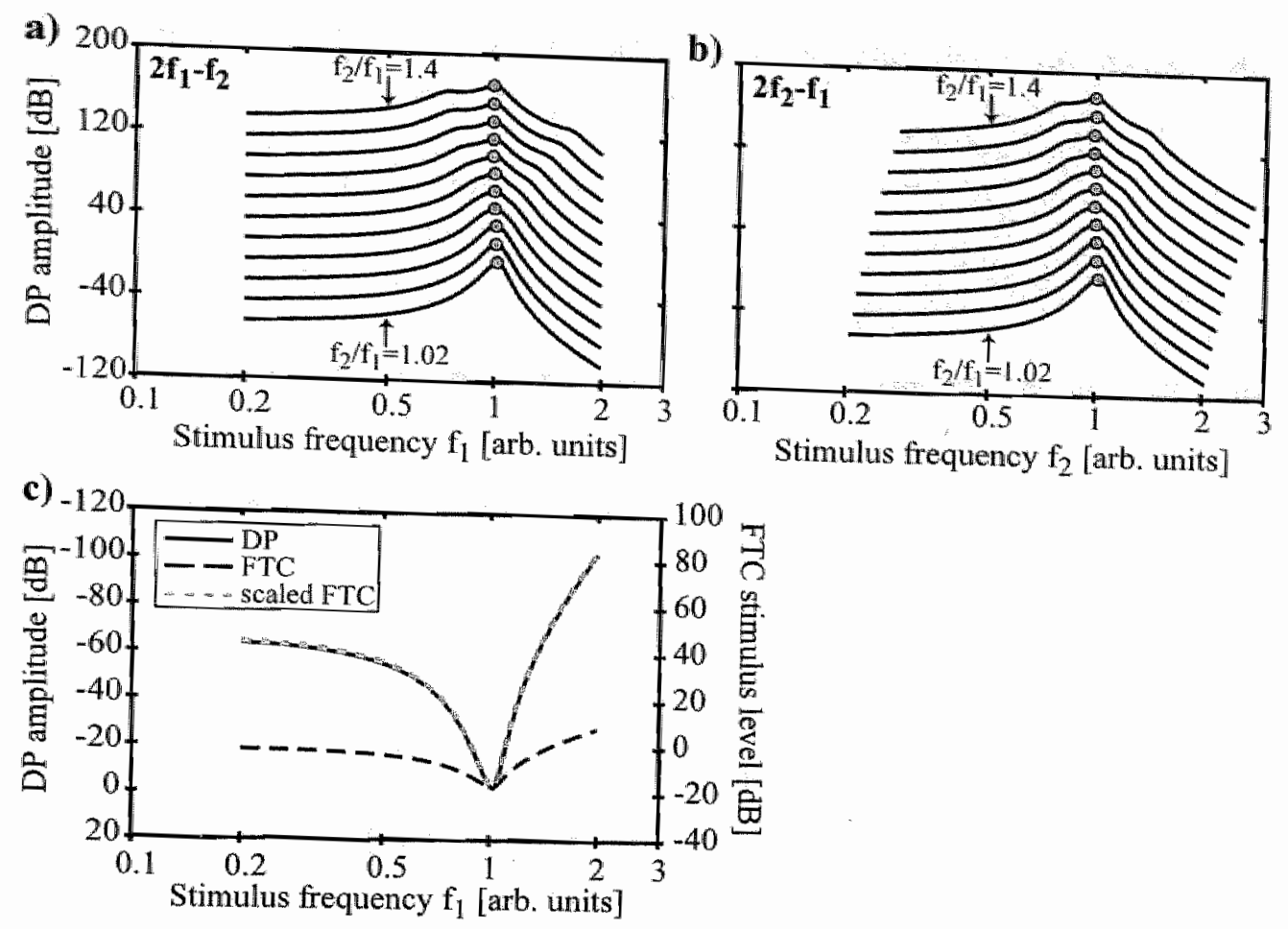

Figure 5.1: Tuning properties for distortion products and nerve fiber from a Duffing oscillator model (See Eq. 5.1). Results are for $m=1, r=1, k_{0}=4 * \pi^{2}, x_{0}=1$ and $A_{1}=A_{2}=1$. a) DPOAE amplitude for $2 f_{1}-f_{2}$, plotted as a function of $f_{11}$. Fach curve displays a DP-audiogram obtained with a different $f_{2} / f_{1}$. For clarity the curves are offet by $20 \mathrm{~dB}$, with $f_{2} / f_{1}=1.02$ being at the botwom. Subsequent curves represent data obtained with increasing $f_{2} / f_{1}$, with the curve for $f_{2} / f_{1}=1.4$ at the top. The muximum in each DP-audiogram is indicated by the filled circles, b) same as a) but for $2 f_{2}-f_{1}$ plotted as function of $f_{2}$. Each DP-audiogram has its maximum when stimulus frequency $f_{11}\left(\right.$ for $2 f_{1}-f_{2}$ ) or $f_{2}$ (for $2 f_{2}-f_{1}$ ) coincides with the resonance frequency of the Duffing ascillator. c) ITC (black, dashed line) as finction of stimulus frequency. The characteristic frequency $(C F)$ of this curve coincides with the resonant frequency of the oscillator, and as a consequence also with the maximum in the DP-audiograns. For comparison, a single DP-audiogram $\left(2 f_{1}-f_{2}, f_{2} / f_{1}=1.02\right)$ is also shown, but with its y-axis reversed (black, solid line). It can be seen that the tuning of the FTC is much broader than the tuning of the DP-audiogran. This difference is absent when the FTC is scaled by a factor of 4 along the $\mathrm{y}$-axis (gray, dashed line). 
In acidition to the FTC, Fig. 5.1c also shows a DP-audiogram (black solid line; $2 f_{1}-f_{2}$ obtained with $f_{2} / f_{1}=1.02$ ). To facilitate graphical comparison between the two, the DP-audiogram is plotted with a reversed $y$-axis (the left $y$-axis corresponds to the DP-audiogram). The FTC is plotted over the same dB-range (right y-axis) as the DP-audiogram, with its offset chosen to match the minima of the two curves. This shows that the FTC is much more broadly tuned than the DP-audiogram. However, when the FTC is scaled by a factor of 4 along the y-axis (gray dashed line), its shape becomes almost identical to that of the DP-audiogram.

In summary, using the Duffing oscillator as a model for the basilar papilla it is found that the maximum DP amplitude occurs when one of the stimulus tones $\left(f_{1}\right.$ for $2 f_{1}-f_{2}$ and $f_{2}$ for $2 f_{2}-f_{I}$, respectively) coincides with the $C F$ of the $\mathbb{F T C}$. This is independent of the stimulus frequency ratio $f_{2} / f_{1}$ used. Also, the FTC is much more broadly tuned than the DP-andiogram, but this difference disappears when the FTC is scaled by a factor of 4 along the y-axis.

\subsection{Results}

Distortion product otoacoustic emissions (DPOAEs) were obtained for a range of stimulus frequencies $\left(213<f_{1}<2774 \mathrm{~Hz}\right.$ ). As a consequence, DPOAEs were recorded from both the amphibian papilla (AP) and the basilar papilla (BP). It is currently believed that the generation of DPOAEs from both papillae gives rise to the bimodal frequency dependence of emission amplitude in DPOAE-audiograms: in the leopard frog, relatively small emission amplitudes are found when the (stimulus) frequencies are around $1250 \mathrm{~Hz}$, while both higher and lower (stimulus) frequencies result in larger DPOAE amplitudes (e.g. Van Dijk et al., 2003). Following Van Dijk and Manley (2001), we will refer to emissions from the amphibian papilla as APDPOAEs, and those from the basilar papilla as BP-DPOAEs.

The frequency dependence of emission amplitude can be seen in Fig. 5.2, which shows a series of DPOAE-audiograms, each one obtained with a different stimulus frequency ratio $f_{2} / f_{1}$. A relative minimum in emission amplitude is found when either $f_{1}$ (for $2 f_{1}-f_{2}$, Fig. 5.2a) or $f_{2}$ (for $2 f_{2}-f_{1}$, Fig. 5.2b) is approximately $1250 \mathrm{~Hz}$. Both for lower and higher stimulus frequencies, emission amplitude exhibits a relative maximum. The filled circles indicate the maximum DPOAE in the basilar papilla frequency range (stimulus frequencies $>1250 \mathrm{~Hz}$ ) in each DPOAEaudiogram. For increasingly larger stimulus frequency ratios $f_{2} / f_{1}$ (subsequent lines in Fig. 5.2) it can be seen that this maximum occurs for a fixed stimulus frequency (fixed- $f_{1}$ for $2 f_{1}-f_{2}$ and fixed- $f_{2}$ for $2 f_{2}-f_{1}$, respectively). Only for the largest stimulus frequency ratios, the maximum BP-DPOAE is found for somewhat lower stimulus frequencies. In the following, we will refer to the stimulus frequency $\left(f_{1}\right.$ 

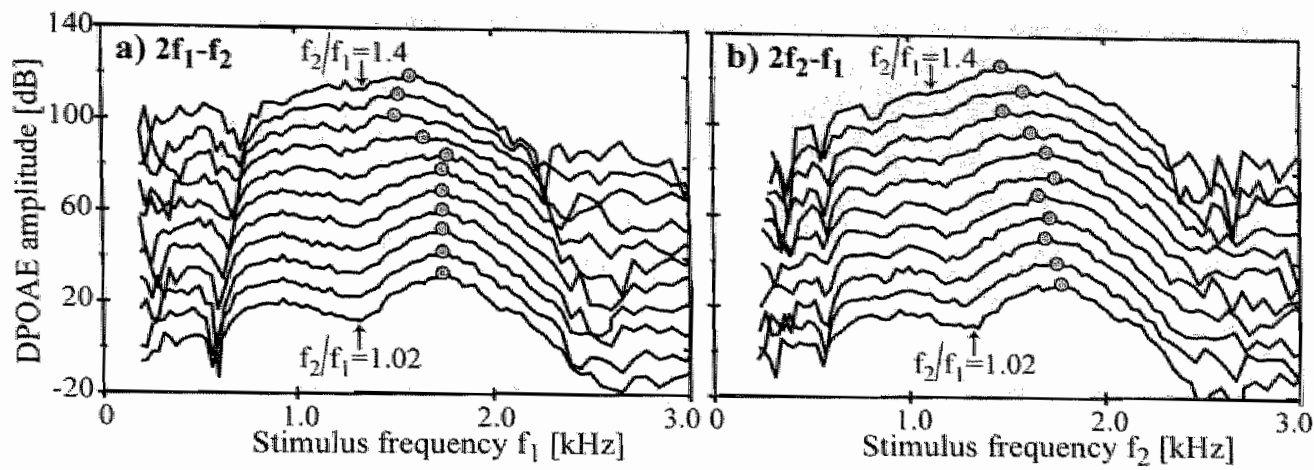

Figure 5.2: Series of DPOAE-audiograms for a) $2 f_{1}-f_{2}$ and b) $2 f_{2}-f_{1}$ obtained in one frog. Each DPOAE-audiogram was recorded by varying the two stimulus tones while keeping the stimulus frequency ratio constant (i.e. fixed- $f_{2} / f_{1}$ sweeps). For darity, curves are offset by $10 \mathrm{~dB}$ with $f_{2} / f_{1}=1.02$ at the bottom and $f_{2} / f_{1}=1.4$ at the top. The stimulus levels were fixed and constant in all sweeps $\left(L_{1}=L_{2}=76 \mathrm{~dB}\right.$ SPL). Notice what for $2 f_{1}-f_{2}$ the stimulus frequency $f_{1}$ is on the abscissa, while for $2 f_{2}-f_{1}$ the stimulus frequency $f_{2}$ is on the abscissa. All DPOA.Es show a bimodal dependence on frequency, with a single relative maximum for frequencies above and below $1250 \mathrm{~Hz}$, respectively. It is currenty believed that this reflects DPOAEs from both the amphibian papilla (APDPOAE, $f<1250 \mathrm{~Hz}$ ) and basilar papilla (BP-DPOAE, $f>1250 \mathrm{~Hz}$ ). For the BP. DPOAE, maxima in emission amplitudes are indicated by the filled circles.

for $2 f_{1}-f_{2}$ and $f_{2}$ for $2 f_{2}-f_{1}$ ) that results in the maximum BP-DPOAE as the distortion product best frequency $B F_{D P}$. The term best frequency $(B F)$ is used, rather than characteristic frequency $(C F)$, since DPOAE-audiograms are equal-input curves. This in contrast to FTCs, which are equal-owtput curves and hence result in characteristic frequencies.

The dependence of $B F_{D P}$ on stimulus frequency ratio $f_{2} / f_{1}$ is further illustrated in Fig. 5.3. This figure shows the shift in $B F_{D P}$ (the shift is relative to $B F_{D P}$ for $f_{2} / f_{1}=1.02$ ) as function of stimulus frequency ratio $f_{2} / f_{1}$, averaged across all frogs. Both for DPOAE at $2 f_{1}-f_{2}\left('+\right.$ ', solid line) and $2 f_{2}-f_{1}(' 0$ ', dashed line), $B F_{D P}$ remains unchanged for relatively small frequency ratios $\left(f_{2} / f_{1}<1.16\right)$. For increasingly larger ratios, BFDP shifts to increasingly lower frequencies.

Besides BP-DPOAEs, we also obtained frequency threshold curves ( $F$ TCs) from each frog. To ascertain that an FTC was obtained from a nerve fiber that innervated the basilar papilla, not only $C F$ and the corresponding threshold level $(C L)$ were determined, but also the bandwidth of the tuning curve at $10 \mathrm{~dB}$ above $C L$ (i.e. $\left.W_{10}\right)$. To distinguish the FTC tuning properties from those calculated for BPDPOAE, we will add the subscript "nerve" to them, rather than the subscript" DP". 


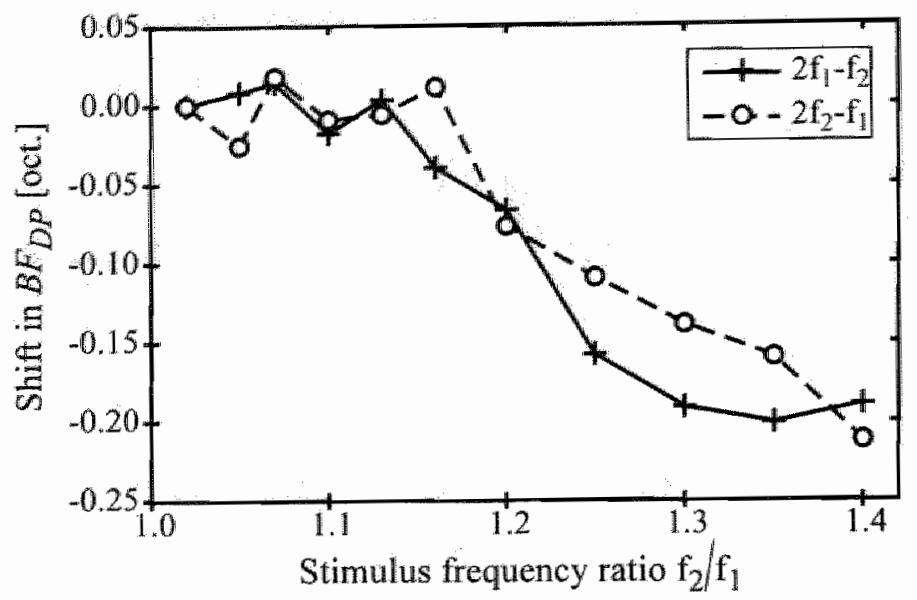

Figure 5.3: The stimulus frequency that resulted in maximum BP-DPOAE $\left(B F_{D P}\right)$ ass a function of the stimulus frequency ratio $f_{2} / f_{1}$, averaged over all frogs. The $B F_{D F}$ found at $f_{2} / f_{1}=1.02$ is taken as a reference, and changes in $B F D P$ are expressed relative to this reference. For neither $2 f_{1}-f_{2}\left({ }^{\prime}+\right.$, solid line) nor $2 f_{2}-f_{1}\left(O^{\prime}\right.$, dashed line) does $B F_{D P}$ change for relatively small stimulus frequency ratios $\left(f_{2} / f_{1}<1.16\right)$. For larger ratios, $B F_{D P}$ shifts towards lower frequencies in both DPOAEs.

Here, $C F_{\text {nerve }}$ ranged from $1464 \mathrm{~Hz}$ to $1949 \mathrm{~Hz}$, while $\left(W_{10}\right)$ merte was between 1272 and $2161 \mathrm{~Hz}$. This latter property indicates that all FTCs were obtained from nerve fibers that innervated the basilar papilla (Ronken, 1991). A graphical comparison between the tuning properties of the FTCs and the DPOAE-audiograms (ine. CF vs. $B F$ and comparison between $C L, W_{10}$, and $Q_{10}=C F / W_{10}$, respectively) revesuled no clear quantitative relation between the two, except when $C F$ and $B F$ are compared (Fig. 5.4). This figure shows $B F_{D P}$ from DPOAE-audiograms obtained

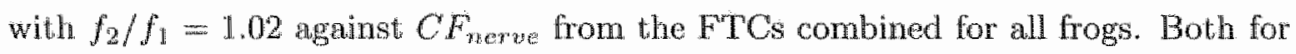
DPOAE at $2 f_{1}-f_{2}$ (filled " 7 ") and $2 f_{2}-f_{1}\left({ }^{*}\right)$ the $B F_{D P}$ roughly coincides with the $C F_{\text {newe }}$ of the accompanying FTC.

Fig. 5.5 shows three examples of $2 f_{1}-f_{2}$ DPOAE-audiograms obtained with $f_{2} / f_{1}=1.02$ (black, solid line) and FTCs (black, dashed line). Each subplot shows data obtained in one frog. To facilitate graphical comparison with the FTC, the DPOAE andiogram is plotted with a reversed $y$-axis (the left $y$-axis corresponds to the DPOAE-amplitude), while the $x$-axis displays stimulus frequency $f_{1}$. The stimulus level range for the FTC (right $y$-axis) was chosen to match the armplitude range used to display the DPOAF-audiogram, while the offset was chosen to match the $C F$ of the FTC and the BF of the (reversed) DPOAE-audiogram. This is the same representation as was used in Fig. 5.1c. All three subplots show that the $C F_{\text {nerve }}$ and 


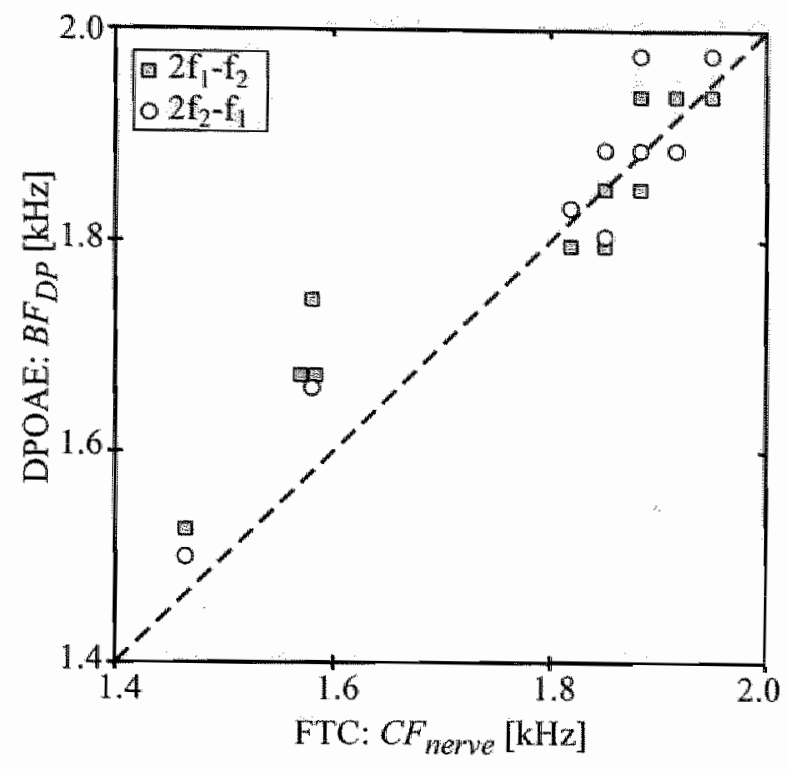

Figure 5.4: Scatter plot of the stimulus frequency resulting in maximum BP-DPOAE amplitude $\left(B F_{D}, p\right)$ for $f_{2} / f_{1}=1.02$ versus the characteristic frequency $\left(C F_{\text {newe }}\right)$ of the nerve fiber FTC. For $2 f_{1}-f_{2}$ (filled ' $\square$ ') $B F$ DP refers to stimulus trequency $f_{1}$, while for $2 f_{2}-f_{1}\left(a^{\prime}\right) B F_{D P}$ refers to stimulus frequency $f_{2}$. As a reference, the diagonal dashed line indicates the condition for which $B F_{D P}=C F_{\text {nerue. }}$.

the $B F_{D P}$ are almost identical, as was already ilustrated in Fig. 5.4. For frequencies away from $C F$, the two curves deviate from each other, resulting in a nuch broader" tuning of the FTC compared to the BP-DPOAE. However, when the FTC is scaled. by a factor of 4 along its $y$-axis (gray dashed lines) the shape of the FTC and the BP-DPOAE become more similar. For frequencies above $C F$, the two curves either partially overlap (Fig. 5.5b), or are oriented approximately parallel (Figs. 5.5a,c). For frequencies below $C F$, the shape of the FTC and the BP-DPOAE are also simIlar. For instance, in the BP-DPOAE of Fig. 5.5a, the relatiwe minimum near 1300 Hz coincides with relatively large DPOAEs at the same stimulus frequency $f_{1}$. In general, the correspondence between the DPOAF-audiogram and the acaled FTC disappears abruptly for frequencies below approximately $1300 \mathrm{~Hz}$. An exception to this is shown in Fig. 5.5c, where the FTC closely follows the DPOAE-audiogram down to a frequency of $1200 \mathrm{~Hz}$. Below $1200 \mathrm{~Hz}$ DPOAFs are generated in the AP, and are not expected to be related to the FTCs obtained from the BP. 

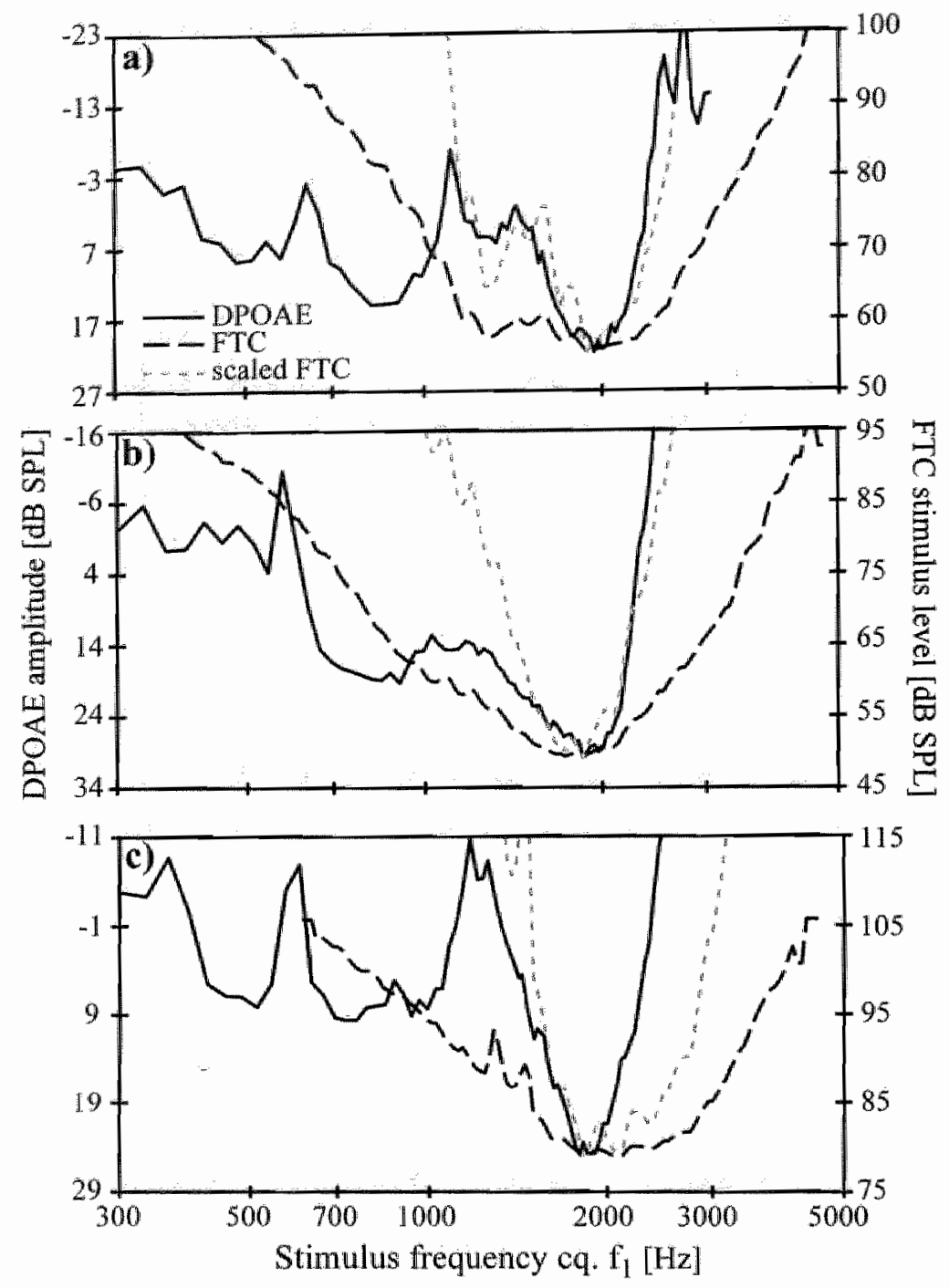

Figure 5.5: Examples of FTCs (black; dashed line) and DPOAE-audiograms for $2 f_{1}-f_{2}$ (black, solid line) recorded in three different frogs. DPOAEs were obtained with $f_{2} / f_{1}=$ 1.02. Each panel has the same general lay-out. The stimulus frequency $f_{1}$ for the DPOAEaudiograms is indicated on the abscissa, while the left y-axis shows the DPOAE amplitude. Notice that this axis goes from high to low levels, resulting in a reversed representation of the DPOA F-audiogram. The right y-axis shows the threshold stimulus levels for the FIC. The range of both $y$-axes are identical, while the offsets were chosen to match the minima of both curves. In each panel, the gray dashed line is the same FTC, but with its $y$-axis scaled by a factor of 4 . 


\subsection{Discussion}

In the present study, we reported on both distortion product otoacoustic emissions (DPOAEs) and nerve fiber frequency threshold curves (FTCs) obtained from the frog basilar papilla (BP). The BP is of interest to hearing research, since it functions as a single auditory filter (Ronken, 1990, Van Dijk and Manley, 2001). This property is not found in any other vertebrate hearing organ. In addition, the tuning properties of nerve fiber FTCs and DPOAEs probably are different manifestations of the same mechanical tuning mechanism present in this papilla, as is implied by the following two considerations (Van Dijk and Manley, 2001): [1] neural tuning of nerve fibers that innervate the basilar papilla presumably originates from a mechanical tuning mechanism (Smotherman and Narins, 1999b). Since this papilla lacks a basilar membrane, or an analogue structure (hair cells are embedded in the rigid wall of the papillar recess), it is reasonable to assume that the mechanical tuning is the result of the mechanics of the hair bundles and the tectorial membrane; [2] BP-DPOAEs are probably generated by the nonlinear mechanics associated with the basilar papilla hair cells and its overlying tectorial membrane (Meenderink and Van Dijk, 2004). Combining these two considerations, one may conclude that the BP-DPOAEs and the neural FTC must reflect the same mechanical filter.

Recently, the Duffing oscillator has been used as a model for DPOAEs from the basilar papilla (Van Dijk and Manley, 2001, Meenderink et al., 2005). These studies show that such a simple model qualitatively describes the dependence of emission amplitude and phase on both absolute $\left(f_{1}, f_{2}\right)$ and relative $\left(f_{2} / f_{1}\right)$ stimulus frequencies. Since tuning in nerve fibers from the basilar papilla arises from the same mechanism as the DPOAE, we also used the Duffing oscillator to model nerve fiber frequency threshold curves. These threshold curves and the tuning properties of the distortion products show several correlations: [1] emission amplitude is maximized whenever the stimulus frequency that is closest to the distortion product frequency (i.e. $f_{1}$ for $2 f_{1}-f_{2}$ and $f_{2}$ for $2 f_{2}-f_{1}$ ) coincides with the resonant frequency of the oscillator. This is independent of the stimulus frequency ratio $f_{2} / f_{1}$ used (Figs. 5.1a,b). The characteristic frequency $(C F)$ of the FTC also coincides with the resonant frequency of the oscillator $(\mathrm{Fig} .5 .1 \mathrm{c})$. Thus, $C F_{\text {zactue }}=B F_{D P}$, independent of the ratio $f_{2} / f_{1}$ used during the DPOAE recordings. [2] The low and high frequency slopes of the DP-audiogram are much steeper than those of the FTC. As a consequence, the FTC is much more broadly tuned than the DP-audiogram. This difference in the shape/slope of the two functions is almost absent when the FTC is scaled by a factor of 4 along the $y$-axis (Fig. 5.10). We will discuss these two model predictions in relation to the BP-DPOAEs and FTC obtained in the frog.

In the simple model, the FTC is inversely proportional to the amplitude response. 


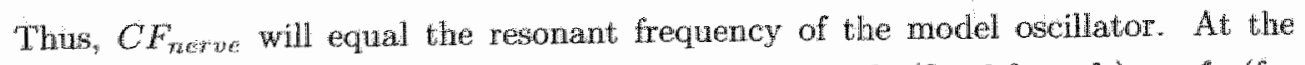
sane time, DP amplitude is naximized when either $f_{1}\left(\right.$ for $2 f_{1}-f_{2}$ ) or $f_{2}$ (for $2 f_{2}-f_{1}$ ) coincides with the resonant frequency. The combination of these two results gives that $B F_{D P}=C F_{\text {werte }}$ independent of the stimulus frequency ratio $f_{2} / f_{1}$. The scatter plot in Fig. 5.4 shows that in the frog $B F_{D P}=C F_{n e r e}$ for $f_{2} / f_{1}=1.02$. As expected from the model, this relation is maintained for increasing $f_{2} / f_{1}$ (as lorg $f_{2} / f_{1}<1.16$ ), since $B F D P$ does not change when the stimulus frequency ratio is increased (Fig. 5.3$)$. However, for larger $f_{2} / f_{1}$ the maxima in the DPOAE-audiograms occur for lower stimulus frequencies, i.e. BFDP decreases, and the predicted relation between $B F_{D P}$ and $C F_{\text {nerve }}$ is no longer found. This discrepancy between the results and the model may be explained by considering the simplifications of the BP when represented by our model.

The Duffing oscillator is intended to model the mechanics of the tectorial membrane and the hair cell stereovilli of the basilar papilla. The oscillator is a simplification of this system in two respects. Firstly, the Duffing oscillator is a second-order system, containing one stiffness element and one mass. In contrast, the basilar papilla contains between 50 -100 hair cells, and the tectorial membrane is a fexible structure with many degrees of freedom. This structure presumably behaves as a high-order system, as is confirmed by recordings from primary auditory nerve fibers (Van Dijk et al., 1994). Secondly, imput-output functions of DPOAEs from the frog ear (Meenderink and Van Dijk, 2004) show that the nonlinear element cannot be as simple as the parabolic stiffness of the Duffing oscillator (Eq. 5.1).

The second result from the model is that the filter shape of the FTC and BPDPOAE are quite different, with the former much mone broadly thmed than the latter. This difference is largely absent (especially around $C F$ ) when the FTC is sculed by a factor of 4 along the $y$-axis. The necessity to scale the FTC by a factor of 4 can be explained by considering the following example. For a given combination of stimulus tones, change stimulus frequency $f_{1}$ in such way that the response of the oscillator (as expressed in $x$ in Eq. 5.1) to this stimulus tone increases by $1 \mathrm{~dB}$. But this shift of $f_{1}$ will also result in a shift of stimulus frequency $f_{2}$. When usiag a small stimulus frequency ratio $f_{2} / f_{1}$, this frequency shift of $f_{2}$ will be approximately the same as the shift for $f_{1}$. At the same time, the small ratio $f_{2} / f_{1}$ ensures that $f_{1}$ and $f_{2}$ change over (approximately) the same absolute frequencies. As a consequence, the change in the movement $x$ in response to the $f_{2}$ frequency-shift will be similar to the change induced by the $f_{1}$ frequency-shift (i.e. $\mathbb{d}$ dB). For a power-law nonlinearity, such $1 \mathrm{~dB}$ increase in both of the stimulus tones will result in a distortion product that is increased in amplitude by $3 \mathrm{~dB}$. This alone, would give rise to a scaling factor of 3. But the generated DPOAEs themselves are part of the response $x$, and will be filtered accordingly. As a consequence, the DPOAE amplitude also changes by 
about $1 \mathrm{~dB}$. Combined, the $3 \mathrm{~dB}$ increase (caused by the $1 \mathrm{~dB}$ increase from the shift of the stimulus frequencies) and the $1 \mathrm{~dB}$ increase (due to the change in DPOAE frequency) result in a scaling factior of 4 .

From Fig. 5.5 it can be seen that after scaling of the FTC by this factor of 4 it partly overlaps with the BP-DPOAE. This is clearest for stimulus frequencies that are close to $C F$. But also for higher stimulus frequencies, the shape of the BP-DPOAE is very similar to the scaled FTC, even th the curves themselves do not overlap (Fig. 5.5c). For frequencies below $C F$, the two curves initially are wery similar. However, when the stimulus frequencies decrease below approximately 1300 $\mathrm{Hz}$, the DPOAE-audiogram shows a sudden change in its shape. This is caused by interference with DPOAEs that originate from the amphibian papilla. It is of interest to see that this sudden deviation between the scaled FTC and the DPOAEaudiogram does not always start at the local minimum of the DPOAE-audiogram (e.g. Fig. 5.5b). This suggests that the transition from BP-DPOAE to AP-DPOAE is not abrupt (at the local minimum in the DPOAE-audiogram), but is more gradual, within a frequency range where AP-DPOAE and BP-DPOAE interfere. In this respect, the data of Fig. $5.5 \mathrm{c}$ are of interest. Here, in the transition from APDPOAE to BP-DPOAE the emissions are indistinguishable from the noise floor for some stimulus frequencies. This suggests that all DPOAEs evoked with higher frequencies are (at least dominated by) BP-DPOAE. In agreement with this, the scaled FTC closely follows the low-frequency slope of the BP-DPOAE down to the noise floor.

\subsection{Conclusions}

We presented both DPOAEs and nerve fiber tuning data from the frog basilar papilla and compared their properties. This comparison is of interest because the BP not only functions as a single auditory filter (Ronken, 1990; Van Dijk and Marley, 2001), it is also believed that both the tuning of nerve fibers (FTC) and BP-DPOAEs are the result of the same mechanical filter (Van Dijk and Manley, 2001) . As a simple model for such a single auditory filter we used the Duffing oscillator. 'This model predicts two correlations between the tuning of DPOAEs and neural tuning. [1] The characteristic frequency of the FTC and the best frequency of BP-DPOAEs will coincide $\left(C F_{\text {nerve }}=B F_{D P}\right)$, independent of the ratio $f_{2} / f_{1}$ used during DPOAE recordings, and [2] the shape of the FTC and the BP-DPOAE will be quite different, with the $\mathbb{F T C}$ much more broadly tuned compared to the BP-DPOAE. This difference in the shape of the two functions largely disappears when the FTC is scaled by a factor of 4 along the $y$-axis. The results obtained from the frog ear agree reasonably well with the model predictions. Only for relatively large stimulus frequency 
ratios evident deviations from the model predictions are observed. These differences presumably result from the oversimplified representation of the $\mathrm{BP}$ by the Duffing oscllator. Notwithstanding these obvious simplifications, the model predicts some of the basic correspondences between FTCs and DPOAE-audiograms from the basilar papila, thus supporting the idea that this hearing organ functions as a single audutory filter.

\subsection{Acknowledgements}

We wish to thank Leola Hau for her assistance in the recording of the frequency threshold curves. This work was supported by grants from the Netherlands Organization for Scientific Research (NWO), and the Heinsius Houbolt Foundation to SWEM and PWD, and NIH grant DC-00222 to PMN. 


\section{Discussion}

In the introduction of this thesis I argued that one of the major tasks of the peripheral auditory system is the spectral decomposition of sound and identified several mechanisms by which this can be achieved. Besides being the result of some of these mechanisms, otoacoustic emissions (OAEs) reflect the in totu response of the auditory system. As such, they can be used to study these spectral-filtering mechanisms non-invasively. The various chapters in this thesis describe the properties of distortion product otoacoustic emissions (DPOAEs) from the anuran ear. In this chapter I will highlight several of these properties and discuss how they can be related to the different filtering mechanisms that operate in the anuran peripheral auditory system.

\section{The two hearing papillae}

It is well known that frequency analysis does occur in the frog ear. The most prominent manifestation of this is the existence of two separate auditory end-organs (papillae) in each ear that are most sensitive to different ranges of auditory frequencies. This initial frequency analysis results in the bimodal shape of DPOAE-audiograms (e.g. Fig. 2.2). Here, DPOAEs with low-frequencies arise from the frog's amphibian papilla (AP), while high-frequency DPOAEs originate from the basilar papilla (BP). Part of this differential frequency response of the two papillae can be ascribed to the impedance imposed by their respective contact membranes on the putative paths of acoustical energy flow within the endolymphatic space (Purgue and Narins, 2000a; $2000 \mathrm{~b})$. In other words, initial spectral decomposition of sound entering the inner ear is realized by the effect that the differential anatomical properties of the papillar recesses and the contact membranes have on the (frequency-dependent) resistance of the different acoustical pathways from the periotic cistern to the round window. At least within the $\mathrm{AP}$, further spectral decomposition of sound occurs, resulting in tonotopic organization of this papilla. For the BP it is not entirely clear whether it holds additional frequency-selective mechanisms, although there are several lines of evidence that suggest there aren't. 


\section{The traveling wave}

It in currenty well established that the mammalian cochlea exhibits tonotopic orgar nization (Von Békésy, 1960). This tonotopy arises from mass and stiffness gradients along the basilar membrane. The resulting macromechanical frequency selectivity (Fay and Popper, 2000 ) can be observed by measuring the mechanical response of the basilar menbrane (e.g. Ruggero and Rich, 1991). One of the most intriguing manifestations of this macromechanical frequency selectivity is the occurrence of traveling waves on the basilar membrane (Von Békésy, 1960).

Since the AP is also tonotopically organized, with the highest sensitivity for different frequencies at different locations along the sensory epithelium (Lewis et al. 1982), the question arises whether this papilla also supports traveling waves. Based on neurophysiological data from the tree frog Eleutherodactylus coqui (Hillery and. Narins, 1984) the presence of traveling waves in the AP has been proposed. Since the AP lacks a basilar membrane or homologue structure, these supposed traveling waves are thought to be in the tectorial membrane (TM) that overlies the sensory epithelium. However, the absence of appropriate mass and stiffness gradients within the TM (or in other inner ear structures) seem to exclude a traveling wave in the AP that originates from macromechanical frequency selectivity (Lewis and Leverenz, 1983). Our extensive exploration of DPOAEs within $\left(f_{1}, f_{2}\right)$ area maps also did not support the notion of mammalign-like traveling waves in the AP (chapter 1). Our results were mankedly different from those obtained in mammals (Kuight and Kemp, 2000; Schneider et al., 2003). These latter results could be explained by a transmission-line model that gives rise to traveling waves (Knight and Kemp, 2001), while the results from the AP were similar to those from the BP, which could be modeled by a single auditory filter.

Therefore, it is likely that the observed tonotopy in the AP does not originate from any macromechanical frequency selective mechanism. Rather, structural differences in the hair cells' mechanical (i.e. micromechanical) and electrical filtering may be the origin of tonotopy. Appropriate combinations of these filtering mechanisms may give rise to a "virtual traveling wave" (Lewis, 1992) with phase-delay characteristics similan" as would be observed in a "genuine" macromechanically-induced, traveling wave. Definite confimation of traveling waves in the AP's tectorial membrane nust await more elaborate recordings of the mechanical response of this structure than are currently available.

A tantalizing question is whether the presence or absence of traveling waves is of any interest. Acoustical energy is not transferred from base to apex by, nor does tonotopy originate from, traveling waves. Rather, traveling waves are a manifestation of tonotopy when the frequency-selectivity arises from a macromechanical 
filtering mechanism. By themselves, they do not contribute to the spectral decomposition or propagation of sound within the inner ear and as such may be considered merely an epiphenomenon.

\section{The inner ear amplifier}

Since the discovery of OAEs it is thought that sound-induced movements of imerear structures are actively enhanced by hair cell reverse transduction (i.e. electromechanical transduction). The presence of an inner-ear amplification mechanism was already proposed by Gold (1948), three decades before the first recordings of OAEs were made (Kemp, 1978). Gold pointed ont that the frequency-selectivity of (auditory) filters decreases when dampening increases. When considering the viscosity of the inner ear fuids, the frequency-selectivity of mammalian auditory-filters would be much less than was observed in experiment, and he realized that this sharper frequency-selectivity could not arise from a passive ear. But the inclusion of a positive feedback loop (connecting the filter's output to its input) in the detec tion system would increase the frequency selectivity of the auditory filter. Such a mechanism needs to compensate for dampening-induced energy losses in the inner ear by supplying additional energy to the system, i.e. it requires an amplifier. Since energy is added, this feedback results in an active filter.

Besides the presence of OAEs, several observations support the involvement of an amplifier in the ear's transduction of sound. Firstly, the auditory system will exhibit higher sensitivity and frequency selectivity than expected by its passive mechanical properties alone. Secondly, the response of the system will grow compressively, being higlily amplified for low-intensity stimuli and less so when stimulus intensities increase. Thirdly, the inner ear amplifier supplies additional energy to the system. In biological systems, this energy is available from biochemical sources which will make the amplifier (and heace both OAE-generation and frequency-selectivity) highly susceptible to various forms of physical, physiological, and chemical insults.

Spontaneous otoacoustic emissions (SOAEs) are the only type of OAEs whose mere occurrence may be interpreted as evidence for the presence of an inner ear amplifier. In the frog, SOAEs have been measured only within the frequency range corresponding to the AP (Van Dijk and Manley, 2001). By itself, this suggests that. an amplification process may be present in this papilla but not; in the BP. Several differences in the DPOAEs from the AP and the BP support this observation. Only DPOAE I/O curves from the AP exhibit compressive growth at low stimulus levels (chapters 2, 3). Furthermore, low-level AP-DPOAEs are much more sensitive to physiological insults (Van Dijk et al., 2003) and changes in temperature (chapter 4) than low-level BP-DPOAEs. 
All evidence, therefore, suggests that an amplification process may be present in the frog $A P$, but not in the BP. This may seem puzzling, since amplification increases the selectivity (and sensitivity) of the detection system. But the absence of an amplifier from the $\mathrm{BP}$ may be beneficial to the frog. The poikilothermic nature of frogs causes their bodies to undergo relatively large temperature fluctuations, resulting in a variable functioning of the inner ear amplifier. With the BP being a passive organ, such temperature fluctuations would not result in a spectral shift of spectral auditory information from this hearing organ, since its proper functioning does not involve any active mechanisms.

Although specifying the exact location and the underlying molecular motor of a possible AP amplifier is not essential to the question whether or not amplification occurs in the frog ear, some speculations can be made. According to general consensus, the inner ear amplifier is located within the sensory hair cells. In mammals, a likely candidate for the cochlear amplifier has been identified in the outer hair cells (OHCs). These cells contain within their lateral membrane a protein (prestin) that is able to deform by voltage-mediated conformational changes (Santos-Sacchi, 1991). This deformation changes the OHC shape in synchrony with an incoming stimulus, providing a cycle-by-cycle amplification of the stimulus-induced $\mathrm{OHC}$ movement (Zheng et al., 2000). Nonmammalian vertebrates, on the other hand, have no OHCs and there is no evidence of, nor likelihood for, somatic motility of their hair cells (Manley, 2001). An alternative amplification mechanism has been suggested in the oscillation of hair cell stereovillar bundles, which has been observed in vitro in several nonmammalian vestibular organs (review in Fudspeth, 1997), the hearing organ of turtles (Crawford and Fettiplace, 1985), and in wivo in the bobtail lizard (Manley et al, 2001). Indirect evidence suggests that the mammalian hair cells stereovillar bundles can also oscillate (Kennedy et al, 2003), while prestin-lacking mice still produce DPOAEs that seemingly arise from an active mechanism (Liberman et al, 2004). To date it is not clear whether or not these mechanisms should be considered alternative lines in the evolution of the inner ear.

\section{The single auditory filter}

Within this thesis, the anuran ear was chosen as the model to study the generation of distortion product otoacoustic emissions. One of the primary motivations for this choice was the notion that in frogs the basilar papilla functions as a single auditory filter (Ronken, 1990; Van Dijk and Manley, 2001). This notion is based on the simple anatomy and physiology of the BP. In ranid frogs, for example, the entire BP contains between 50 and 100 hair cells, with their stereovillar bundles oriented identically (Lewis, 1978). Also, in an individual frog almost all BP nerve 
fibers are tuned to the same frequency and have identically shaped neural tuning curves (Ronken, 1990; Van Dijk et al., 1997). In addition, the hair cells in the BP are not innervated by efferent nerve fibers, thus excluding (complex) feedback mechanisms from the central nerwous system. Although the precise mechanisms of frequency-selectivity are not completely understood, these seem to be of mechanical origin, without the (additional) contribution of electrical tuning from the hair cells (Smotherman and Narins, 1999a).

This unique, single-filter property of the BP would allow, with relative ease, the characterization of the frequency-filtering mechanisms that result in the generation of DPOAEs from this papilla. This in contrast to for example the mammalian cochlea, where the interpretation of DPOAEs is much complicated by the compound effects of (many) adjacent micromechanical auditory filters. Throughout this thesis we used this property to model the BP using a simple, single oscillator (the Duffing oscillator) and showed that this model qualitatively describes the DPOAE-dependence on both the absolute $\left(f_{2}, f_{1}\right)$ and relative $\left(f_{2} / f_{1}\right)$ stimulus frequencies (chapter 1) as well as several correlations between neural and DPOAE-tuning (chapter 5 ). These findings, combined with other DPOAE properties (e.g. chapter 3), provide additional support that the BP indeed functions as a single auditory filter.

With the Duffing oscillator as a simple model for the $\mathrm{BP}$, the next step seems to be to correlate the various elements of the model to specific anatomical structures within the BP, thus pinpointing the mechanisms underlying the generation of DPOAEs. Besides the difficulty in doing this, there are several arguments why this may not be appropriate. Firstly, any nonlinear resonator that is tuned to a single frequency gives rise to frequency-related characteristics similar to those found in the frog, r.e. this is not limited to the Duffing oscillator only. This may indicate that the observed patterns in DPOAEs and the correlations between DPOAE and neural tuning merely reftect aspects of the single-frequency tuning of the BP and not the underlying DPOAE generation mechanisms. Secondly, the observed level-dependent characteristics of DPOAEs (chapters 2, 3) are rather difficult to reproduce using the Duffing oscillator (or any sidgle nonlinear oscillator model), while it seems that these characteristics are most suitable to study the underlying. DPOAE-gencration. mechanisms. Again, this indicates that the model itsell does not give an accurate description of the nonlinear mechanisms from which DPOA Es originate. However; these latter mechanisms can be modeled by nonlinearities that exhibit no freguencyselectivity (chapter 3 ). It seems that the BP stould be described by a composite model, with elements describing the frequency-selectivity of the papilla (perhaps from tuning by the tectorial membrane) and the nonlinearities within the papilla that result in the generation of DPOAEs (hair cells and/or hair cell-tectorial membrane interactions). 
Finally, even with the successfnl formulation of such a model, it is questionable whether it can be extended to describe the single filter-elements within the ears of other tetrapods. As sad, active processes play a key role in the observed frequencyselectivity of auditory organs. Therefore, any model that accurately describes the involved filtering mechanismis in these ears must include this amplification. But across tetrapods, the frog $\mathrm{BP}$ seems to be the only auditory organ that lacks such an amplification mechanism (t.e. chapter 4) disallowing the direct extrapolation of any BP-rrodel to the tetrapod ear. Notwithstanding this, the BP seems to most simple vertebrate anditory system, and knowledge about its functioning will contribute to the general understanding of vertebrate hearing. In addition, the AP does seem to inclucle some amplification mechanism, similar as in other vertebrates. Since the structure of the AP is still rellatively simple (i.e. no basilar membrane) the interpretation of OAEs in relation to these mechanisms may be more straightforward. Seemingly, this knowledge can be directly extrapolated to vertebrate hearing.

In summary, the anuran inner ear holds two auditory papillae. Although the BP may be considered 'too simple' to represent vertebrate hearing, understanding of its functioning can form the base for more elaborate models that describe auditory processing in vertebrates. At the same time, the frog AP does seem suitable to function as a model for active vertebrate hearing, with the benefit that its anatomy (and functioning) is rellative simple within all vertebrate auditory end-organs. Combined, understanding the functioning of the anuran inner ear may significantly contribute to understanding the universal mechanisms of auditory processing in vertebrates. 


\section{Bibliography}

Abdala, C. (2000) Distortion product otoacoustic emission $\left(2 f_{1}-f_{2}\right)$ amplitude growth in human adults and neonates. J. Acoust. Soc. Am. 107, 446-456.

Bregman, A. S. (1990) Auditory scene analysis: The perceptual organization of sound. MIT Press, Cambridge, MA.

Brown. A. M. (1987) Acoustic distortion from rodent ears: A comparison of responses from rats, guinea pigs, and gerbils. Hear. Res. 31, 25-38.

Brown, A. M., Harris, F. P., and Beveridge, H. A. (1996) Two sources of acoustic distortion products from the human cochlea. J. Acoust. Soc. Am. 100, 3260-3267.

Brownell, W. E., Bader, C. R., Bertrand, D., and de Ribaupierre, Y. (1985) Evoked mechanical responses of isolated cochlear outer hair cells. Science 227, 194-196.

Capranica, R. R. and Moffat, A. J. M. (1975) Selectivity of the peripheral auditory system of spadefoot toads (Scaphiopus couchi) for sounds of biological significance. J. Comp. Physiol. 100, 231-249.

Coro, F. and Kössl, M. (2001) Components of the $2 f_{1}-f_{2}$ distortion-product otoacoustic emission in a moth. Hear. Res. 162, 126-133.

Crawford, A. C., Evans, M. G., and Fettiplace, R. (1989) Activation and adaptation of transducer currents in turtle hair cells. J. Physiol. 419, 405-434.

Crawford, A. C. and Fettiplace, R. (1985) The mechanical properties of ciliary bundles of turtle cochlear hair cells. J. Physiol. 364, 359-379.

Davis, H. (1983) An active process in cochlear mechanics. Hear. Res. 9, 79-90.

Dorn, P. A., Konrad-Martin, D., Neely, S. T., Keefe, D. H., Cyr, E, and Gorga, M. P. (2001) Distortion product otoacoustic enission input/output functions in normal-hearing and hearing-impaired human ears. J. Acoust. Soc. Am. 110, 3119-3131.

Dreisbach, L. E. and Siegel, J. H. (2001) Distortion-product otoacoustic emissions measured at high frequencies in humans. J. Acoust. Soc. Am. 110, 2456-2469.

Evans, E. F. (1979) Single unit studies of mammalian cochlear nerve. In: H. A. Beagley (Ed.), Auditory investigations: The scientific and technological basis, Clarendon Press, Oxford, pp. 324-367.

Fay, R. R. and Popper, A. N. (2000) Evolution of hearing in vertebrates: The inner ear processing. Hear. Res' 149, 1-10.

Feng, A. S., Narins, P. M., and Capranica, R. R. (1975) Three populations of primary auditory nerve fibers in the bullfrog (Rana catesbeina): Their peripheral origins and frequency sensitivities. J. Comp. Physiol. 100, 221-229.

Ferber-Viart, C., Savourey, G., Garcia, C., Duclaux, R., Bittel, J., and Collet, L. 
(1995) Influence of hyperthermia on cochlear micromechanical properties in humans. Hear. Res. 91, 202-207.

Frishkopf, L. S., Capranica, R. R., and Goldstein, M. H. (1968) Neural coding in the bullfrog's auditory system-a teleological approach. Proc. IEEE 56, 969-980. Frishkopf, L. S. and Flock, A. (1974) Ultrastructure of the basilar papilla, an auditory organ in the bullifrog. Acta Otolaryngol. 77, 176-184.

Frishkopf, L. S. and Goldstein, M. H. (1963) Responses to acoustic stimuli from single units in the eight nerve of the bullfrog. J. Acoust. Soc. Am. 35, 1219-1228.

Frolenkov, G. I., Belyartseva, I. A., Kurc, M., Mastroianni, M. A., and Kachar, B. (1998) Cochlear outer hair cell electromotility can provide force for both low and high.. intensity distortion product otoactoustic emissions. Hear. Res. 126, 67-74. Froymovich, O., Rebala, V., Salvi, R. J., and Rassael, H. (1995) Long-term effect of acoustic trauma on distortion product otoacoustic emissions in chickens. $J$. Acoust. Soc. Am. 97, 3021-3029.

Gaskill, S. A. and Brown, A. M. (1990) The behavior of acoustic distortion product, $2 f_{1}-f_{2}$, from the human ear and its relation to auditory sensitivity. J. Acoust. Soc. Am. 88, 821-839.

Geàl-Dor, M., Khvoles, R, and Sohmer, H. (1997) Cooling induces a decrease in middle ear compliance. J. Basic Clin. Physiol. Pharmacol. 8, 127-132.

Gold, T. (1948) Hearing II. the physical basis of the action of the cochlea. Proc. $\mathrm{R}$. Soc. E. B135, 492-498.

He, D. Z., Beisel, K. W., Chen, L., Ding, D. L., Jia, S., Fritzsch, B., and Salvi, R. (2003) Chick hair cells do not exhibit voltage-dependent somatic motility. J. Physiol. 546, 511-520.

Hillery, C. M. and Narins, P. M. (1984) Neurophysiological evidence for a traveling: wave in the amphibian inner ear. Science 225, 1037-1039.

Hillery, C. M. and Narins, P. M. (1987) Frequency and time domain comparison of low-frequency auditory fiber responses in two anuran amphibians. Hear. Res, 25, 233-248.

Howard, J. and Hudspeth, A. J. (1988) Compliance of the hair bundle associated with gating of mechanoelectrical transduction channels in the bullfrog's saccular hair cell. Neurom. 1, 189-199.

Hudspeth, A. J. (1989) How the ear's works work. Nature 341, 397-404.

Hudspeth, A. J. (1997) Mechanical amplification of stimuli by hair cells. Current Opinion in Neurobiology $7,480-486$.

Kemp, D. T. (1978) Stimulated acoustic emissions from within the human auditory system. J. Acoust. Soc. Am. 64, 1386-1391.

Kemp, D. T. (1979) Evidence of mechanical nonlinearity and frequency selective wave amplification in the cochlea. Arch. Otorhinolaryngol. 224, 37-45. 
Kemp, D. T. and Brown, A. M. (1983) An integrated view of cochlear mechanical nonlinearities observable from the ear canal. In: E. de Boer and M. A. Viergever (Eds.), Cochlear Mechanics, Delft University Press, Delft; pp. 75-82.

Kennedy, H. J., Evans, M. G., Crawford, A. C., and Fettiplace, R. (2003) Fast adaptation of mechanoelectrical transducer channels in mammalian cochlear hair cells. Nature Neurosci. 6, 832-836.

Kettembeil, S., Manley, G. A., and Siegl, E. (1995) Distortion-product otoacoustic emissions and their anesthesia sensitivity in the European Starling and the chicken. Hear. Res. 86, 47-62.

Khvoles, R., Freeman, S., and Solmmer, H. (1998) Effect of temperature on the transient evoked and distortion product otoacoustic emissions in rats. Audiol. Neurootol. 3, 349-360.

Kim, D. O. (1980) Cochlear mechanics: Implications of electrophysiological and acoustical observations. Hear. Res. 2, 297-317.

Knight, R. D. and Kemp, D. T. (2000) Indications of different distortion product otoacoustic emission mechanisms from a detailed $f_{1}, f_{2}$ area study. J. Acoust. Soc. Am. 107, 457-473.

Knight, R. D. and Kemp, D. T. (2001) Wave and place fixed DPOAE maps of the human ear. J. Acoust. Soc. Am. 109, 1513-1525.

Kummer, P., Janssen, T., Hulin, P, and Arnold, W. (2000) Optimal $L_{1}-L_{2}$ primary tone level separation remains independent of test frequency in humans. Hear. Res. $146,47-56$.

Lewis, E. R. (1978) Comparative studies of the anuran auditory papillae. Scan. Electron Microsc. II, 633-642.

Lewis, E. R. (1984) On the frog amphibian papilla. Scan. Electron Microsc. IV, $1899-1913$.

Lewis, E. R. (1992) Convergence of design in vertebrate acoustic sensors. Iri: D. B. Webster, R. R. Fay, and A. N. Popper (Eds.), The Evolutionary Biology of Hearing, Springer-Verlag, New York, pp. 163-184.

Lewis, E. R. and Leverenz, E. L. (1983) Morphological basis for tonotopy in the anuran amphibian papilla. Scan. Electron Microsc. I, 189200.

Lewis, E. R., Leverenz, E. L., and Koyama, H. (1982) The tonotopic organization of the bullfrog amphibian papilla, an auditory organ lacking a basilar membrane. J. Comp. Physiol. 145, 437-445.

Lewis, E. R. and Narins, P. M. (1999) The acoustic periphery of amphibians. In: R. R. Fay and A. N. Popper (Eds.), Comparative Hearing: Fish and Amphibians, Springer-Verlag, New York, pp. 101-154.

Liberman, M. C., Gao, J., He, D. Z. Z., Wu, X., Jia, S., and Zuo, J. (2002) Prestin is required for electromotility of the outer hair cell and for the cochlear amplifier. 
Nature $419,300-304$

Liberman; M. C., Zuo, J., and Guinan, J. J. (2004) Otoacoustic emissions without somatic motility: Can stereocilia mechanics drive the mammalian cochlea? J. Acoust. Soc. Am. 116, 16491655 .

Lonsbury-Martin, B. L., Harris, F. P., Stagner, B. B., Hawkins, M. D., and Martin, G. KK. (1990) Distortion product emissions in humans. I. Basic properties in nomally hearing subjects. Ann. Otol. Rhinol. Laryngol. Suppl. 147, 3-14.

Lonsbury.Martin, B. L., Martin, G. K., Probst, R., and Coats, A. C. (1987) Acoustic distortion products in rabbit ear canal I. Basic features and physiological vulnerability. Hear. Res. $28,173-189$.

Lukashkin, A. N., Lukashkina, V. A., and Russell, I. J. (2002) One source for distorthon product otoacoustic emissions generated by low- and high-level primaries. J. Acoust. Soc. Am. 111, 2740-2748.

Lukashtin, A. N. and Russell, I. J. (1998) A descriptive model of the receptor potential nonlinearities generated by the hair cell mechenoelectrical transducer. J. Acoust. Soc. Am. 1.03, 973-980.

Lukashkin, A. N. and Russell, I. J. (1999) Analysis of the $f_{2}-f_{1}$ and $2 f_{1}-f_{2}$ distortion components generated by the hair cell mechanoelectrical transducer: Dependence on the amplitudes of the primaries and feedback gain. J. Acoust. Soc. Am. 106, 2661-2668.

Manley, G. A. (1986) The evolution of the mechanisms of frequency selectivity in vertebrates. In: B. C. J. Moore and R. D. Patterson (Eds.), Auditory Frequency Selectivity, Plenum Press, New York, pp. 63-72.

Manley, G. A. (1990) Peripheral hearing mechanisms in reptiles and birds. SpringerVerlag, Berlin.

Manley, G. A. (2000) Otoacoustic emissions in lizards. In: G. A. Manley, H. Fastl, M. Kösı1, H. Oeckinghaus, and G. M. Klump (Eds.), Auditory worlds: Sensory analysis and perception in animals and man, Wiley-VHC, Weinheim, pp. 93102 . Manley, G. A. (2001) Evidence for an active process and a cochlear amplifier in nommanmals. J. Neurophysiol. $86,541-549$.

Mantey, G. A. (2002) Evolution of structure and function of the hearing organ in lizards. J. Nentobiol. 53, 202-211.

Manley, G. A., Köppl, C., and Johnstone, B. M. (1993) Distortion-product otoacoustic emissions in the bobtail lizard. I. General characteristics. J. Acoust. Soc. Am. 93, 2820-2833.

Manley, G. A., Yates, G. K., Kirk, D. L., and Köppl, G. (2001) In-vivo evidence for a cochlear amplifier in the hair-cell bundle of lizards. Proc. Nati. Acad. Sci. USA. $98,2826-2831$.

Manley, G. A., Yates, G. K., and Koppl, G. (1988) Auditory peripheral tuning: 
evidence for a simple resonance phenomenon in the lizard Tiliqua. Hear. Res. 33 , 181-190.

Manley, G. A., Yates, G. K., Köppl, C, and Johnstone, B. M. (1990) Peripheral auditory processing in the bobtail lizard Tiliqua rugosa. IV. Phase locking of auditory-nerve fibres. J. Comp. Physiol. A 167, 129-138.

Meenderink, S. W. F., Narins, P. M., and Van Dijk, P. (2005) Detailed $f_{1}, f_{2}$ area study of distortion product otoacoustic emissions in the frog. J. Assoc. Res. Otolaryngol. $6,37-47$.

Meenderink, S. W. F. and Van Dijk, P. (2004) Level dependence of distortion product otoacoustic emissions in the leopard frog, Rana pupiens piptens. Hear. Res. 192, $107-118$.

Mills, D. M. (1997) Interpretation of distortion product otoacoustic emission measurements. I. Two stimulus tones. J. Acoust. Soc. Am. 102, 413-429.

Mills, D. M. (2002) Interpretation of standard distortion product otoacoustic emission measurements in light of the complete parametric response. J. Acoust. Soc. Am. 112, 1545-1560.

Mills, D. M., Norton, S. J., and Rubel, E. W. (1993) Vulnerability and adaptation of distortion product otoacoustic emissions to endocochlear potential variation. J. Acoust: Soc. Am. 94, 2108-2122.

Mills, D. M. and Rubel, E. W. (1994) Variation of distortion product otoacoustic emissions with furosemide injection. Hear. Res. 77, 183-199.

Mills, D. M. and Rubel, E. W. (1996) Development of the cochlear amplifier. J. Acoust. Soc. Am. 100, 428-441.

Mom, T., Bonfils, P., Gilain, L., and Avan, P. (2001) Origin of cubic difference tones generated by high-intensity stimuli: effect of ischemia and auditory fatigue on the gerbil cochlea. J. Acoust. Soc. Am. 110, 1477-1488.

Narins, P. M. and Hillery, C. M. (1983) Frequency coding in the inner eas of anuran amphibians. In: R. Klinke and R. Hartmann (Eds.), Hearing-Physiological bases and Psychophysics, Springer-Verlag, Heidelberg, pp. 70-76.

Noyes, W. S., McCaffrey, T. V., Fabry, D. A., Robinette, M. S., and Suman, V. J. (1996) Effect of temperature elevation on rabbit cochlear function as measured by distortion-product otoacoustic emissions. Otolaryngol. Head Neck Surg. 115, $548-552$.

Patuzzi, R. (1996) Cochlear micromechanics and macromechanics. In: P. Dallos, A. N. Popper, and R. R. Fay (Eds.), The Cochlea, Springer-Verlag, New York, pp. $186-257$.

Pibal, I., Drexl, M., and Kössl, M. (2002) Level dependence of optimal stimulus level difference for evoking DPOAEs in the gerbil. Hear. Res. 174, 260 263.

Purgue, A. P. and Narins, P. M. (2000a) Mechanics of the inner ear of the bullfrog 
(Rana catesbetana): The contact membranes and the periotic canal. J. Comp. Physiol. A $186,481-488$.

Pargue, A. P. and Narins, P. M. (2000b) A model for energy flow in the inner ear of the bullfog (Rona catesbeiana). J. Comp. Physiol. A 186, 489-495.

Robbins, R. G., Banknight, B. S., and Honrubia, M. D. (1967) Anatonical disuribution of the efferent fibers in the VHIth cranial nerve of the bullrog Rana catesbezana. Acta Otolaryngol. 64, 436-448.

Ronken, D. A. (1990) Basic properties of auditory-nerve responses from a 'simple' ear: The basilar papilla of the frog. Hear. Res. 47, 63-82.

Ronken, D. A. (1991) Spike discharge properties that are related to the characteristic frequency of single units in the frog auditory nerve. J. Acoust. Soc. Am. 90, 24282440 .

Rosowski, J. J., Peake, W. T., and White, J. R. (1984) Cochlear nonlinearities from two-tone distortion products in the ear canal of the alligator lizard. Hear. Res. $13,141-158$.

Ruggero, M. A. and Rich, N.C. (1991) Application of a commercially-manufactured Doppler-shift laser velocimeter to the measurement of the basilar-membrane vibration. Hear. Res. 51, 215-230.

Santos-Sacchi, J. (1991) Reversible inhibition of voltage-dependent outer hair cell. motility and capacitance. J. Neurosci. 11, 3096-3110.

Schneider, S., Prijs, V. F., and Schoonhoven, R. (2003) Amplitude and phase of distortion product otoacoustic emissions in the guinea pig in an $\left(f_{1}, f_{2}\right)$ area study. J. Acoust. Soc. Am. 113, 3285-3296.

Shera, C. A and Guinan, I. J. (1999) Evoked otoacoustic emissions arise by two fundamentally different mechanisms: A taxonomy for mammalian OAEs. J. Acoust. Soc. Am. 105, 782-798.

Smotherman, M. S. and Narins, P. M. (1999a) The alectrical properties of auditory hail cells in the frog amphibian papilla. J. Neurosci. 19, 5275-5292.

Smotherman, M. S. and Narins, P. M. (1999b) Potassium currents in auditory hair cells of the frog basilar papilla. Hear. Res. 132, 117-130.

Sticbler, 1. B. and Narins, P. M. (1990) Temperature-dependence of auditory nerve response properties in the frog. Hear. Res. 46, 63-81.

Stover, L. J., Neely, S. T. and Gorga, M. P. (1996) Latency and multiple sources of distortion product otoacoustic emissions. J. Acoust. Soc. Am. 99, 1016-1024.

Sun, H., Salvi, R. I., Ding, D. L., Hashino, E., Shero, M, and Zheng, X. Y. (2000) Excitotoxic effect of kamic acid on chicken otoacoustic emissions and cochlear potentials. J. Acoust. Soc. Am. 107, 2136-2142.

Talmadge, C. L., Long, G. R., Tubis, A., and Dhar, S. (1999) Experimental confirmation of the two-source interference model for the fine structure of distortion. 
product otoacoustic emissions. J. Acoust. Soc. Am. 105, 275-292.

Talmadge, C. L., Tubis, A., Long, G. R. and Piskorski, P. (1998) Modeling otoacoustic emission and hearing treshold fine structures. J. Acoust. Soc. Am. 104, $1517-1543$.

Taschenberger, G. and Manley, G. A. (1998) General characteristics and suppression tuning properties of the distortion-product otoacoustic emission $2 f_{1}-f_{2}$ in the barn owl. Hear. Res. 123, 183-200.

Tonndorf, J. (1981) Physiological Acoustics. Hutchinson Ross Pub., Stroudsburg.

Van Dijk. P., Lewis, E. R., and Wit, H. P. (1990) Temperature effects on auditory nerve fiber response in the american bullfrog. Hear. Res. 44, 231240.

Van Dijk, P. and Manley, G. A. (2001) Distortion product otoacoustic emissions in the tree frog Hyla cinerea. Hear. Res. 153, 14-22.

Van Dijk, P., Mason, M. J., and Narins, P. M. (2002) Distortion product otoacoustic emissions in frogs: correlation with middle and inner ear properties. Hear. Res. $173,100-108$.

Van Dijk, P., Narins, P. M., and Mason, M. J. (2003) Physiological vulnerability of distortion product otoacoustic emissions from the amphibian ear. J. Acoust. Soc. Am. 11.4, 2044-2048.

Van Dijk, P., Wit, H. P., and Segenhout, J. M. (1989) Spontaneous otoacoustic emissions in the European edible frog (Rana esculenta): Spectral details and temperature dependence. Hear. Res. 42, 273-282.

Van Dijk, P., Wit, H. P., and Segenhout, J. M. (1997) Dissecting the frog inner ear with Gaussian noise. I. Application of high-order Wiener-kemel analysis. Hear. Res. $114,229-242$.

Van Dijk, P., Wit, H. P., Segenhout, J. M., and Tubis, A. (1994) Wiener kemel analysis of imner ear function in the American bullfrog. J. Acoust. Soc. Am.95, $904-919$.

Veuillet, E., Gartner, M., Champsaur, G., Neidecker, J., and Collet, L. (1997) Effects of hypothermia on cochlear micromechanical properties in humans. J. Neurol. Sci. $145,69-76$.

Von Békésy, G. (1960) Experiments in Hearing. McGraw-Hill, New Yort.

Wever, E. G. (1973) The ear and hearing in the frog, Rana pipiens. J. Morphol. 141, $461-478$.

Wever, E. G. (1985) The amphibian ear. Princeton University Press, New Jersey.

Whitehead, M. L., Lonsbury-Martin, B. L., and Martin, G. K. (1992) Fvidence for two discrete sources of $2 f_{1}-f_{2}$ distortion-product otoacoustic emission in rabbit: I. Differential dependence on stimulus parameters. 1. Acoust. Soc. Am. 91. $1587-1607$.

Whitehead, M. L., Stagner, B. B., McCoy, M. I., Lonsbury-Martin, B. I., and 
(1995) Dependence of distortion-product otoacoustic emissions on sin normal and impaired ears. II. Asymmetry in $L_{1}, L_{2}$ space. J. Am. 97, 23592377 .

Narins, P. M. (1985) Temporary threshold shift, adaptation, and acteristics in frog auditory nerve fibers. Hear. Res. 17, 161-176.

W., He, D. Z., Long, K. B., and Dallos, P. (2000) Prestin is the เ of cochlear outer hair cells. Nature 405, 149-155.

urk, W., and Kim, D. O. (1982) The behavior of acoustic distortion te ear canals of chinchillas with normal or damaged ears. J. Acoust. $774-780$. 


\section{Samenvatting}

\section{Distorsieproduct otoakoestische emissies uit het kikkeroor}

"Daf alle unsere Erkenntnis mit der Erfahrung anfange, daran ist gar kein Zweifel" (Kant, I.)

Kennis van de wereld om ons heen begint met de waarneming ervan. Met betrekking tot de waarneming van geluid is er het oor. Simpel gezegd dient het oor om mee te horen. Maar wat is horen? Wat is het dat we willen waamemen met ons gehoon? Een van de manieren om deze vraag te beantwoorden ligt gevat in het concept: auditory scene analysis". Hervan is het idee dat het gehoor wordt gebruikt om een cognitief beeld op te bouwen van de akoestische wereld orn ons been, min of meer analoog aan de opbouw van de visuele wereld via onze ogen. Bimnen dit concept zijn de bepaling van de locatie en de identificatie van de verschillende geluidstronnen van eminent belang. Met andere woorden, het gaat erom dat je met je oren kunt. vaststellen wax je wat hoort.

De bepaling van wat je hoort hangt samen met het vaststellen van de fysische eigenschappen van geluid; de frekwentie en de amplitude. De eerste stap in de identificatie van deze fysische eigenschappen vindt plaats in het perifeer-gelegen gedeelte van het oor. Bijvoorbeeld, in zoogdieren worden gelujden met verschillende frekwenties op verschillende plaatsen in het slakkenhuis omgezet in zenuwactiviteit, resulterend in een plaats-trekwentie relatie (de tonotopische organisatie) langs het basilair membram. Tegenwoordig zijn ex verschillende mechanismen bekend die cen rol spelen bij deze eerste analyse van het binnenkomende geluid. De werking wan deze mechanismen resulteert in een scala wan verschillende fenomenen, waarwan éui de in dit proefschrift beschrewen otoakoestische emissies (OAEs) zijn.

In 1978 werd voor het eerst beschreven dat gehuid iniet alleen het oor ingaxt, max dat het zelf ook in staat is om geluid te produceren. Deze geluiden knnnen worden. gemeten als OAEs door een gevoelige microfoon aan te sluiten op het oor. Ondanks de initiele scepsis over het bestaan van deze "oorgeluiden" vormen ze tegenwoordig een belangrijk instrument bimen het gehooronderzoek, juist omdat hun generatie samen lijkt te hangen met die mechanismen die essenticel zijn voor het normaal functioneren wan het oor. Dit laatste blijkt uit de bevinding dat OAEs alleen worden gemeten in het normal functionerende oor; de aanwezigheid wan een gehoorveriles leidt wrijwel altijd tot het verdwijnen van de emissies. Hiemaast blijki dat niet alloen 
in zoogdieren, maar in alle op het land levende gewervelde dieren OAEs kunnen worden gemeten. Kennelijk zijn de mechanismen die ten grondslag liggen aan de generatie van OADs universeel voorkomende gehoorsmechanusmen.

Otoakoustische emissies kunnen worden onderverdeeld in een aantal categorieen. De eerite onderverdeling is in spontane en opgewekte OAEs, warbij de eerstgenoemde categorie geen externe stinulus vereist en de laatste categorie wel. Afhankelijle van de gebruikte stimulus wordt een verdere vardeling van de opgewekte OAEs gemakt. Wanneer de stimullus bestaat ut twee zuivere tonen dan genereert het binnenoor distorsieproduct otoakoestische emissies (DPOAEs). Dize DPOAEs vallen samen met de intermodulatie-frekwenties van de twee stimulus tonen; als de stimulus frekwenties worden genoteerd met $f_{1}$ en $f_{2}$ dan worden de DPOAE-frekwenties gegeven door $n f_{1}-m f_{2}$, waarbij $2 f_{1}-f_{2}$ over het algemeen de luidste is.

Gezien de oorsprong wan OAEs is het mogelijk deze geluiden te gebruiken om de achterliggende gehoorsmechanismen te bestuderen. Hierbij is het een voordeel dat emissies zeer eenvoudig gemeten kunnen worden; het aansluiten van een microfoon op het oor is voldoende. Er zijn dus geen ingewikkelde en beschadigende ingrepen noodzakelijk om de metingen te kunnen verrichten. Fen nadeel van deze niet-invasieve meetmethode is dat OAEs een weerspiegeling zijn wan het gehele oor. De karakteristieken van emissies zijn derhalwe tweeledig. Enerzijds weerspiegelen ze hun generatie-mechanismen, welke waarschijnlijk gelijk zijn aan essentiële gehoorsmechanismen in het binnenoor. Anderzijds worden deze eerstgenoemde karakteristieken gemoduleerd door de in totu respons van het complete on. Vanwege deze tweeledigheid in de emissie-karakteristieken gebruik ik de termen "micromechanismen" en "macro-mechanismen" on onderscheid te maken tussen de mechanismen die de emissies genereren en de mechanismen die de samengestelde respons var het gehele oor beschrijven. De hedendaggse anname is dat de generatiemechanismen wan OAEs in alle dieren identiek zijn; de met de harcellen geassociearde micromechanismen variëren schjubaar niet tussen de verschillende klassen van gewervelde dieren. Echter, de macro-mechanismen hangen nauw samen met do anatomie van het oor warabinen de hearcellen zijn ingebed. Deze anatomie vertoont een grote variatie tussen de verschilende kJassen van gewervelde dieren, net als gevolg dat de macromechanismen eveneens variëren.

De anatomie van het kikkeroor is relatief simpel ten opzichte van het slakkenhuis zoals dat wordt gevonden in zoogdieren. Ondanks deze eenvoudige bouw zijn de cellen die tem grondslag liggen aan de generatie van OAEs, de laarcellen, min of meer identiek aan die in zoogdieren. In plaats van een slakkenhuis bevinden zich in heli, oor van de kikker twee, anatomisch gescheiden, papillae die gevoelig zijn voor geluid. De amfibische papilla (AP) is het orgaan dat het meest gevoelig is voor de laagste en de middelste frekwenties binnen het gehoorsbereik van de kikker, terwijl 
de basilaire papilla (BP) het meest gevoelig is voor de hoogste frekwenties binnen dit bereik. Van deze papillae is de BP de meest eenvoudige. Het sensorische epithelium van deze papilla bevat "slechts" 50 tot 100 haarcellen. Verder zijn de "tuning"karakteristieken van wrijwel alle zenuwvezels die deze haarcellen innerveren identiek. Het lijkt erop dat deze gehele papilla functioneert als één auditief filter. Hoewel de bouw van de AP complexer is dan die van de BP, is deze nog altijd eenvoudiger dan het slakkenhuis. Hierbij is in mijn ogen een belangrijk verschil met het slakkenhuis dat de haarcellen in de AP niet zijn gepositioneerd op een flexibel membraan, maar direct zijn ingebed in de kraakbenige structuur van het binnenoor. Dit heeft tot gevolg dat de koppeling tussen de opeenvolgende micro-mechanische filterelementen fundamenteel anders moet zijn. Terwijl in het slakkenhuis deze koppeling voornamelijk gerealiseerd wordt door drukwerschillen tussen verschillende scalae (en resulteert in zogenaamde "lopende golven") is dit type koppeling in het kikkeroor onmogelijk. De afwijkende anatomie van beide papillae zal leiden tot andere macromechanismen dan in het slakkenhuis. Zoals gezegd drukken deze mechanismen ook hun stempel op de karakteristieken van de gegenereerde emissies. Vanwege de relatief simpele bouw van beide papillae in het kikkeroor is de verwachting dat deze macro-mechanische beïnloeding relatief eenvoudig te duiden zal zijn.

Dit proefschrift beschrijft in vijf hoofdstukken verschillende experimenten waarin distorsieproduct otoakoestische emissies zijn gemeten in de kikker. Allereerst blijkt het dat beide papillae in staat zijn DPOAEs te genereren. Omdat de papillae het: meest gevoelig zijn voor geluid van verschillende frekwenties, en derhalve emissies genereren over verschillende frekwentie-bereiken (zie Figs. 3.2a,b), is het mogelijk elke papilla min of meer onafhankelijk van de andere te bestuderen. Bij de interpretatie van de gemeten DPOAEs uit elke papilla is het van behang het hierboven beschreven tweeledige karakter van emissies voor ogen te houden. Het blijkt dat de micro-mechanismen, die ten grondslag liggen van de emissie-generatie, hei best. bestudeerd kunnen worden door variatie in de luidheid van de twee stimulus tonen (zie Hfst. 2,3 en 4). Dit soort experimenten levert zogeneamde input/outputfuncties (I/O-functies) op, waarbij de luidheid van de stimulus tonen (de inpul) wordt gerelateerd aan de luidheid van de resulterende DPOAEs (de output; zie Hfst. 2). Deze 1/O-functies laten zien dat bij de generatie van DPOAEs twee verschillende micro-mechanismen betrokken zijn, waarbij elk mechanisme een eigen emissiecomponent veroorzaakt. De luidheid van beide stimulus tonen bepaalt, welke component de gemeten emissies domineert. Voor relatief zachte ("low-level") stimulus tonen is het generatie-mechanisme een verzadigende niet-lineariteit, terwijl voor relatief luide ("high-level") stimulus tonen dit mechanisme wordt beschreven door een niet-verzadigende niet-lineariteit. 
Hoewel dit beeld wan twee enissie-componenten van toepassing is op beide papil how, is or wet ean opvallend verschil in de low-level component van elke papilla. Voor toenemende lwidheid van de stimulus tonen neemt de luidheid wan de low-level component in de amfibische papilla toe met maximaal $1 \mathrm{~dB} / \mathrm{dB}$, min of meer identiek arn de situatie in het slakkenhus. In de basilaire papilla is deze toename duidelik groter, tussen de 2 en $3 \mathrm{~dB} / \mathrm{dB}$. Een mogelijlke verklaring voor dit verschil is de aanwezighetel van een zogenaamde "cochleaire wersterker" in de amfibische papilla. Deze versterket zorgt erwor dat de door het geluid opgewekte trillingen in het bimnenoor worden versterkt. Naast een toename in de gevoeligheid en selectiviteit van het oor, resulteert een dergeljke versterker ook in de wargenomen relatief langzame groei van de DPOAE luidheid. De relatief snelle groei van DPOAEs in de basilaite papilla suggereert dat in deze papilla geen versterker werkzam is. Om dit te testen hebben we experimenten gedaan waarbij de lichaamstemperatuur van de kilkker werd gevarieerd (zie HIst. 4). Hierbij is het idee dat de versterker energie verbruikt. Voor lage temperaturen is deze energite niet voorhanden en zal de verstenker niet functioneren. Zoals verwacht vonden we dat low-level DPOAEs uit de amfibische papilla aftankelijk zijin van de lichamstemperatuur, terwijl DPOAEs uit: de basilaire papilla hierwar onaflankelijk waren. De bevinding dat de amfibische papilla een versterker gebruikt is niet uniek, de aanwezigheid vari een versterker in het slakkenhuis was al lang vermoed. Tot op heden zijn er zelker twee verschillende moleculaire mechanismen geidentificeerd die als versterker kunnen functioneren. Uniek is wel dat; de basilaire papilla geen versterker bexat. Kennelijk bereikt deze papilla zijn gevoeligheid op een andere manier dan via signaalversterking. De afwezigheid van een temperatuur-afhankelijke versterker kan zelfs een voordeel zijh voor de kikker. Aangezien de lichaanstemperatuur van "koudbloedige" dieren alluangt van de omgevingstemperatuur, lan deze aanzienlijk variëren. Met het temperatuur=onafhankelijk functioneren van de basilaire papilla resulteren deze tomperatuuschommelingen niet in variatie in cle spectrale output van dit gehoororgaain.

In tegenstelling tot de micro-mechanismen blijkt voor de bestudering van de macro-mechanismen systematische variatie in de frekwenties van beide stimulus tonen het meest geschikt (zie Hfst. 1), hoewel sommige I/O-karakteristieken ook het gevolg zijn wan deze macromechanismen (zie Hfst. 3). Gebaseerd op de bevinding dat de basilaire papilla functioneert als één auditief filter hebben we cen simpel model gebruikt om de frekwentie-afluanklijkheid van DPOAEs uit deze papilla te simuleren. Hierbij vonden we een kwalitatief goede overeenkomst tussen de experimentele en moclel-resultaten, wat aangeeft dat de basilaire papilla inderdaad functioneert; als een enkelvoudige resonator. Opvallend was dat de frekwentie-afhankelijkheid van DPOAEs in de amfibische papilla in essentie gelijk is aan die in de basilaire 
papilla. Dit suggereert dat deze papilla, net zoals de BP, gemodelleerd kan worden door één enkele resonator. Deze bevinding lijkt haaks te staan op de aanwezige tonotopische organizatie in de AP. Hoewel tot op heden onduidelijk, ligt een mogelijke verklaring in de koppeling tussen opeenvolgende filter-elementen in de AP. Onze resultaten geven een verschil tussen de AP en het slakkenhuis in deze macromechanische koppeling weer. Zoals gezegd komt in het slakkenhuis deze koppeling tot stand omdat het basilaire membraan (met daarop de hatrcellen) zich tussen twee met vloeistof gevulde scalae bevindt. Door deze vloeistof-koppeling ontstaan er op het basilaire membraan zogenaamde lopende golven die zich manifesteren in specifieke patronen in de DPOAE resultaten. Gezien de anatomie van de amfibische papilla kan de koppeling hier niet op een soortgelijke manier plaativinden, en lijken zulk soort lopende golven in deze papilla niet waarschijnlijk. Hoewel zulke lopende golven zijn voorgestela in de amfibische papilla bieden onze DPOAE-resultaten geen ondersteuning voor deze hypothese.

Hoewel het merendeel van dit proefschrift handelt over DPOAEs, zijn emissies niet de functionele output van het gehoor. Uiteindelijk gaat het erom dat de fysischo eigenschappen van het waargenomen geluid worden doorgegeven aan de hersenen. Deze doorgave gaat via de gehoorzenuw, die een verbincling vormt tussen de haarcellen en de hersenstam. Daar de basilaire papilla functioneert als een enkelvoudig auditief filter, waarbij de "tuning" waarschijnlijk het gevolg is van mechanische eigenschappen, is de verwachting dat er eenvoudige relaties bestaan tussen de "tuning" van DPOAEs en zenuwvezels. Het eerder genoemde model voorspelt een tweetal van deze relaties die we hebben getest door zowel emissies als zenuwvezel responsies te meten (zie Hfst 5). Deze metingen bevestigen de voorspelde relaties, zodoende verder bewijs leverend dat de basilaire papilla inderdaad functioneert als een enkelvoudige, mechanische resonator. 


\section{Curriculum vitae}

Sebastiaan Willem Frederik (Bas) Meenderink was born in Hengelo (The Netherlands) on July 17, 1976. After completing the secondary school at the Bataafse Kamp in 1994 he attented the Wageningen Agricultural University (The Netherlands). In 1999, he obtained a M.Sc. in Biology (specialization theoretical/mathematical Biology) at this university. While awaiting a suitable position to become a Ph.D. candidate, he worked for a year at the Gemeenschappelijk Administratiekantoor (GAK) in Utrecht (The Netherlands).

In October 2000 he joined the Faculty of Medicine at the Maastricht University (The Netherlands) as a Ph.D. candidate under the supervision of Dr. P. van Dijk. The following four years he participated in the NWO-project 'Distortion product otoacoustic emissions from the amphibian inner ear" where he investigated various properties of distortion product otoacoustic emissions in the frog. For this project he also visited the laboratory of Prof. Dr. P.M. Narins at the University of California, Los Angeles (USA) for three months. This doctoral thesis reports on the research. he has carried out within this project to receive his Ph.D. degree. 


\section{List of Publications}

Meenderink, S.W.F, Narins, P.M., and Van Dijk, P. (2005) Detailed $f_{1}, f_{2}$ area study of distortion product otoacoustic emissions in the frog. J. Assoc. Res. Otolaryngol. 6, 37-47.

Meenderink, S.W.F., and Van Dijk, P. (2004) Level dependence of distortion product otoacoustic emissions in the leopard frog, Rana pipiens pipiens. Hear. Res. $192,107-118$.

Meenderink, S.W.F., and Van Dijk, P. (accepted) Characteristics of distortion product otoacoustic ernissions in the frog from $L_{1}, L_{2}$-maps. J. Acoust. Soc. Am.

Meenderink, S.W.F., and Van Dijk, P. (in preparation) The effect of body-temperature on distortion product otoacoustic emissions in the frog.

Meenderink, S.W.F., Van Dijk, P., and Narins, P.M. (accepted) Comparison between distortion product otoacoustic emissions and nerve fiber responses from the basilar papilla of the frog. J. Acoust. Soc. Am.

Simmons, D.D., Meenderink, S.W.F., and Vassilakis, P. (submitted) Eight nerve/ Inner ear/ Otoacoustic emissions. In: P.M. Narins, and A.S. Feng (Eds.). Hearing and sound communication in amphibians, Springer-Verlag, New York.

Van Dijk, P., and Meenderink, S.W.F. (submitted) Distortion product otoacoustic emissions in the amphibian ear. In: Auditory mechanisms: processes and models, $9^{\text {th }}$ "Mechanics of Hearing" Workshop, Portland, USA, $23^{\text {rd }}-28^{\text {th }}$ July 2005, World Scientific Publishing Co.

Vassilakis, P., Meenderink, S.W.F., and Narins, P.M. (2004) Distortion product otoacoustic emissions provide clues to hearing mechanisms in the frog ear. J. Acoust. Soc. Am. 116, 3713-3726. 